\title{
Immunological Evaluation of Synthetic Glycosylphosphatidylinositol Glycoconjugates as Vaccine Candidates Against Malaria
}

Ankita Malik, ${ }^{1,2,3, \S}$ Fridolin Steinbeis, ${ }^{1,4, \S}$ Maria Antonietta Carillo, ${ }^{1}$ Peter H. Seeberger, ${ }^{1,2}$ Bernd Lepenies ${ }^{1,2,5 *}$ and Daniel Varón Silva ${ }^{1,2 *}$

${ }^{1}$ Department of Biomolecular Systems, Max Planck Institute of Colloids and Interfaces, Am Mühlenberg 1, 14476 Potsdam, Germany

${ }^{2}$ Institute of Chemistry and Biochemistry, Freie Universität Berlin, Arnimallee 22, 14195 Berlin, Germany

${ }^{3}$ Current address: Institute of Supramolecular Science and Engineering, Allée Gaspard Monge 8, Strasbourg FR 67083, France

${ }^{4}$ Current address: Department of Infectious Diseases and Respiratory Medicine, Charité Universitätsmedizin Berlin, Charitéplatz 1, 10117 Berlin, Germany

${ }^{5}$ Immunology Unit \& Research Center for Emerging Infections and Zoonoses, University of Veterinary Medicine Hannover, Bünteweg 17, 30559 Hannover, Germany.

$\S$ Contributed equally to this work

* Corresponding author(s)

\section{Supporting Information}

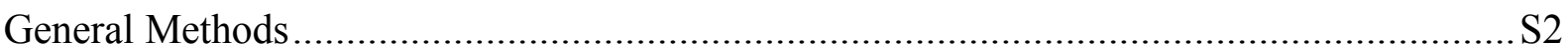

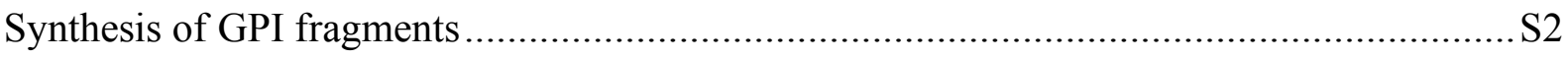

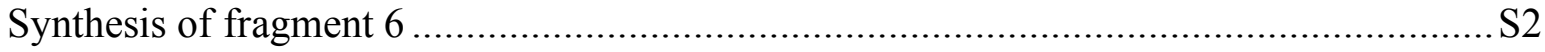

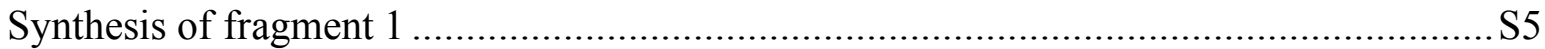

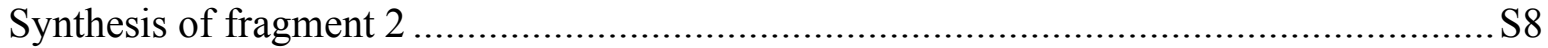

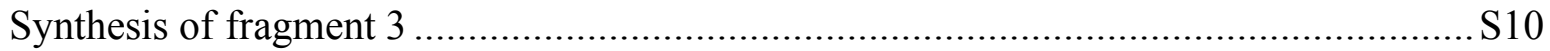

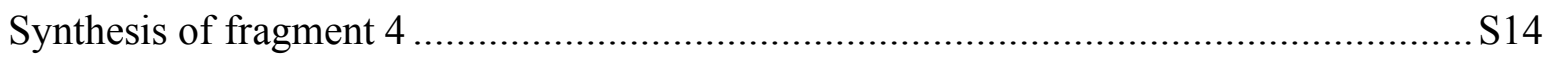

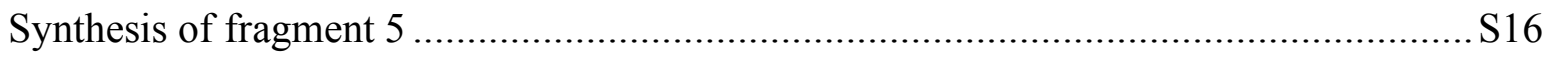

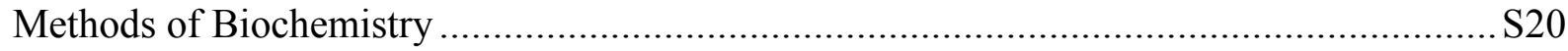

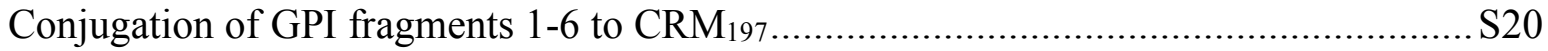

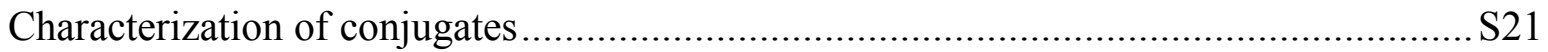

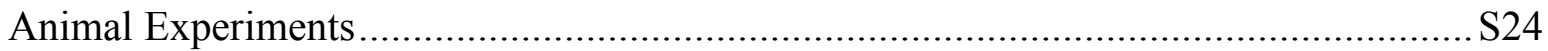

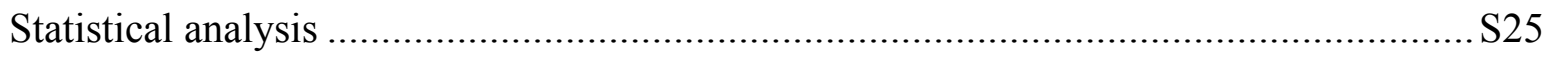

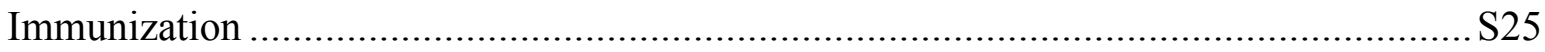

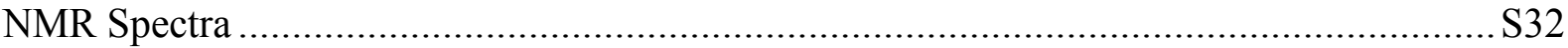

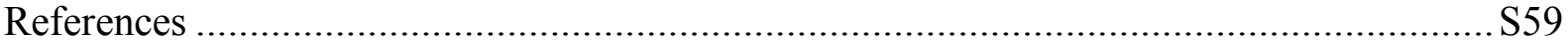




\section{General Methods}

All purchased chemicals were of reagent grade and all anhydrous solvents were of high-purity grade and used as supplied except where noted otherwise. Reactions were performed in ovendried glassware under an inert argon atmosphere unless noted otherwise. Reagent grade thiophene was dried over activated molecular sieves prior to use. Pyridine was distilled over $\mathrm{CaH}_{2}$ prior to use. Sodium hydride suspension was washed with hexane and THF and stored in an anhydrous environment. Benzyl bromide was passed through activated basic aluminum oxide prior to use. Metal sodium was washed with hexane and stored in hexane. Analytical thin layer chromatography (TLC) was performed on Merck silica gel $60 \mathrm{~F}_{254}$ plates $(0.25 \mathrm{~mm})$. Compounds were visualized by UV irradiation or heating the plate after dipping in staining solution. The staining solutions were cerium sulfate-ammonium molybdate (CAM) solution, basic potassium permanganate solution, acidic ninhydrin-acetone solution, or a 3methoxyphenol-sulfuric acid solution (Sugar Stain). Flash column chromatography was carried out using a forced flow of the indicated solvent on Sigma Aldrich silica gel high purity grade $60 \AA$ (230-400 mesh particle size, for preparative column chromatography). Solvents were removed under reduced pressure using rotary evaporator and high vacuum $(<1 \mathrm{mbar})$. Freeze drying of the aqueous solutions was performed using Alpha 2-4 LD Lyophilizer (Christ, Osterode am Harz, Germany)

${ }^{1} \mathrm{H},{ }^{13} \mathrm{C}$ and ${ }^{31} \mathrm{P}-\mathrm{NMR}$ as well as all 2D-spectra $\left({ }^{1} \mathrm{H}-{ }^{1} \mathrm{H}\right.$ COSY, ${ }^{1} \mathrm{H}-{ }^{1} \mathrm{H}$ TOCSY, ${ }^{1} \mathrm{H}-{ }^{13} \mathrm{C}$ HSQC, $\left.{ }^{1} \mathrm{H}-{ }^{13} \mathrm{C} \mathrm{HMBC}\right)$ were recorded on a Varian $400(400 \mathrm{MHz})$, a Varian $600(600 \mathrm{MHz})$, a Bruker $400(400 \mathrm{MHz})$ and a Bruker Ascend $400(400 \mathrm{MHz})$ spectrometer in $\mathrm{CDCl}_{3}\left(7.26 \mathrm{ppm}{ }^{1} \mathrm{H}\right.$, $\left.77.1 \mathrm{ppm}{ }^{13} \mathrm{C}\right), \mathrm{D}_{2} \mathrm{O}\left(4.79 \mathrm{ppm}{ }^{1} \mathrm{H}\right), \mathrm{MeOD}\left(4.87 \mathrm{ppm}\right.$ and $3.31 \mathrm{ppm}{ }^{1} \mathrm{H}, 49.00 \mathrm{ppm}{ }^{13} \mathrm{C}$ ), Acetone- $\mathrm{d}_{6}\left(2.05 \mathrm{ppm}\right.$ and $2.84 \mathrm{ppm}{ }^{1} \mathrm{H}, 206.26 \mathrm{ppm}$ and $\left.29.84 \mathrm{ppm}{ }^{13} \mathrm{C}\right)$ unless otherwise stated. The coupling constants $(J)$ are reported in Hertz $(\mathrm{Hz})$. Splitting patterns are indicated as s, singlet; d, doublet; t, triplet; q, quartet; br, broad singlet; dd, doublet of doublets; m, multiplet; $\mathrm{dt}$, doublet of triplets; $\mathrm{h}$, hextet for ${ }^{1} \mathrm{H}$ NMR data. Signals were assigned by means of ${ }^{1} \mathrm{H}-{ }^{1} \mathrm{H}$ COSY, ${ }^{1} \mathrm{H}_{-}{ }^{1} \mathrm{H}$ TOCSY, ${ }^{1} \mathrm{H}_{-}{ }^{13} \mathrm{C}$ HSQC,${ }^{1} \mathrm{H}_{-}{ }^{13} \mathrm{C}$ HMBC spectra and version thereof. ESI mass analyses were performed by Waters Xevo G2-XS Q-TOF with an Acquity H-class UPLC and a Bruker Autoflex-speed MALDI-TOF spectrometer.

\section{Synthesis of GPI fragments}

\section{Synthesis of fragment 6}

The fragment $\mathbf{6}$ and all mannose building blocks were synthesized according to the previously published protocols. ${ }^{1,2}$

\section{Synthesis of mannose $\alpha-(1 \rightarrow 4)$ glucosamine disaccharide}

The mannose $\alpha-(1 \rightarrow 4)$ glucosamine disaccharide (10) is present in the fragments ( 1 to 4$)$ and was used as a common building block to obtain the fragments via a convergent approach involving a [2+2] or a [2+3] glycosylation. $\alpha-(1 \rightarrow 2)$-dimannose and $\alpha-(1 \rightarrow 2)$-trimannose were synthesized using the established protocol. ${ }^{3}$ 


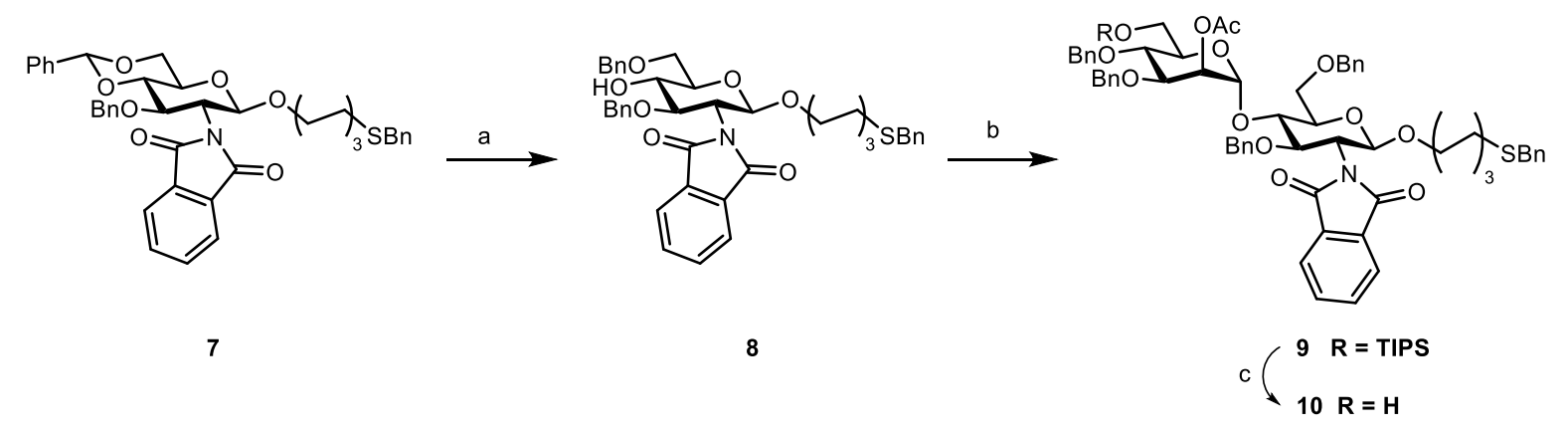

Scheme S1: Synthesis of pseudo-disaccharide 10. a) TFAA, TFA, TES, $\mathrm{CH}_{2} \mathrm{Cl}_{2}, 0{ }^{\circ} \mathrm{C}, 5 \mathrm{~h}, 80 \%$; b) 26, TMSOTf, $\mathrm{CH}_{2} \mathrm{Cl}_{2},-40{ }^{\circ} \mathrm{C}$ to $-20{ }^{\circ} \mathrm{C}, 1 \mathrm{~h}, 88 \%$; c) HF-py, THF, rt, $48 \mathrm{~h}, 83 \%$.

1-O-(6-thiobenzyl)hexyl-3,6-O-benzyl-2-deoxy-2-N-phthalimido- $\beta$-D-glucopyranoside (8)

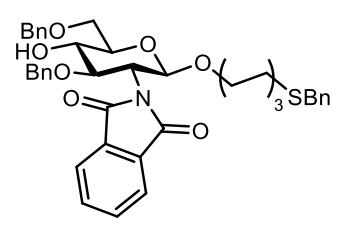

To a stirred solution of $7^{4}(0.5 \mathrm{~g}, 0.72 \mathrm{mmol})$ in $\mathrm{CH}_{2} \mathrm{Cl}_{2}(8 \mathrm{~mL})$ were added trifluoroacetic anhydride $(0.31 \mathrm{~mL}, 2.16 \mathrm{mmol})$ and triethylsilane $(0.58 \mathrm{~mL}, 3.6 \mathrm{mmol})$ were added at $0{ }^{\circ} \mathrm{C}$ followed by trifluoroacetic acid $(0.28 \mathrm{~mL}, 3.6 \mathrm{mmol})$ drop wise. The reaction mixture was stirred at $0{ }^{\circ} \mathrm{C}$. After $5 \mathrm{~h}$, the reaction mixture was quenched with $\mathrm{Et}_{3} \mathrm{~N}$ and concentrated to give yellow oil that was purified by flash column chromatography to obtain glucoasamine acceptor $8(0.4 \mathrm{~g}, 0.57 \mathrm{mmol}, 80 \%)$ as a colorless oil. $\mathrm{R}_{f}=0.33$ (Hexanes/EtOAc $\left.=3: 1\right){ }^{1} \mathrm{H}$ NMR $(400$ $\left.\mathrm{MHz}, \mathrm{CDCl}_{3}\right) \delta 7.83-7.60(\mathrm{~m}, 4 \mathrm{H}), 7.39-7.18(\mathrm{~m}, 8 \mathrm{H}), 7.04(\mathrm{dt}, J=6.0,2.2 \mathrm{~Hz}, 2 \mathrm{H}), 6.98-$ $6.90(\mathrm{~m}, 3 \mathrm{H}), 5.28(\mathrm{~s}, 2 \mathrm{H}), 5.10(\mathrm{dd}, J=8.3,3.0 \mathrm{~Hz}, 1 \mathrm{H}), 4.73(\mathrm{dd}, J=12.2,1.6 \mathrm{~Hz}, 1 \mathrm{H}), 4.65$ $-4.54(\mathrm{~m}, 2 \mathrm{H}), 4.52(\mathrm{dd}, J=12.2,1.2 \mathrm{~Hz}, 1 \mathrm{H}), 4.21(\mathrm{ddd}, J=10.8,8.3,1.3 \mathrm{~Hz}, 1 \mathrm{H}), 4.12$ (ddd, $J=10.7,8.3,1.8 \mathrm{~Hz}, 1 \mathrm{H}), 3.85-3.69(\mathrm{~m}, 4 \mathrm{H}), 3.63$ (ddd, $J=9.5,4.9,1.6 \mathrm{~Hz}, 1 \mathrm{H}), 3.60$ (s, $1 \mathrm{H}), 3.38-3.23(\mathrm{~m}, 2 \mathrm{H}), 2.16(\mathrm{t}, J=7.3 \mathrm{~Hz}, 1 \mathrm{H}), 1.44-1.23(\mathrm{~m}, 3 \mathrm{H}), 1.21-0.93(\mathrm{~m}, 5 \mathrm{H})$. ${ }^{13} \mathrm{C}$ NMR $\left(101 \mathrm{MHz}, \mathrm{CDCl}_{3}\right) \delta 138.1,137.5,133.8,128.7,128.5,128.4,128.1,127.9,127.8$, $127.8,127.3,126.8,98.2,78.6,74.6,74.2,73.7,73.3,70.8,69.4,55.3,53.4,44.7,36.1,32.3$, 31.0, 29.0, 28.9, 28.3, 26.3, 25.3, 25.1. ESI-MS $(\mathrm{m} / \mathrm{z}):[\mathrm{M}+\mathrm{Na}]^{+}$cald 718.86 obsd 718.6

2-O-Acetyl-3,4-di-O-benzyl-6-O-triisopropylsilyl- $\alpha$-D-mannopyranosyl-(1 $\rightarrow 4)-1-O-(6-$ thiobenzyl)hexyl-3, 6-O-benzyl-2-deoxy-2-N-phthalimido- $\beta$-D-glucopyranoside (9)

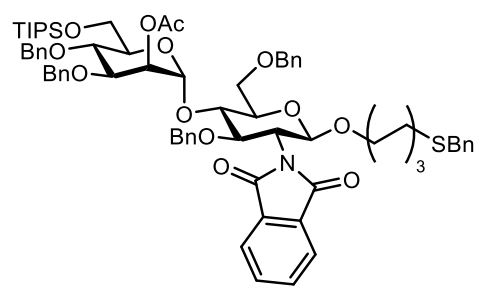

The mannose donor $(0.36 \mathrm{~g}, 0.52 \mathrm{mmol})$ and glucosamine acceptor $8(0.3 \mathrm{~g}, 0.43 \mathrm{mmol})$ were co-evaporated with anhydrous toluene $(3 \times 7 \mathrm{~mL})$ and dried under high vacuum for $2 \mathrm{~h}$. The mixture was dissolved in anhydrous $\mathrm{CH}_{2} \mathrm{Cl}_{2}(8 \mathrm{~mL})$ and activated molecular sieves were added. The solution was stirred for $10 \mathrm{~min}$ at room temperature and cooled to $-40{ }^{\circ} \mathrm{C}$. The mixture was treated with TMSOTf $(19 \mu \mathrm{L}, 0.086 \mathrm{mmol})$ and slowly warmed to $-20{ }^{\circ} \mathrm{C}$ over a period of $1 \mathrm{~h}$. 
The reaction was diluted with $\mathrm{CH}_{2} \mathrm{Cl}_{2}$, quenched with $\mathrm{Et}_{3} \mathrm{~N}$ and concentrated. The crude product was purified by flash column chromatography to obtain disaccharide $9(0.47 \mathrm{~g}, 0.38 \mathrm{mmol}$, $88 \%)$ as white solid. $\mathrm{R}_{f}=0.5($ Hexanes/EtOAc $=3: 1){ }^{1} \mathrm{H}$ NMR $\left(400 \mathrm{MHz}, \mathrm{CDCl}_{3}\right) \delta 7.63(\mathrm{~d}, J$ $=16.0 \mathrm{~Hz}, 5 \mathrm{H}), 7.39-7.17(\mathrm{~m}, 28 \mathrm{H}), 7.00(\mathrm{~d}, J=7.4 \mathrm{~Hz}, 2 \mathrm{H}), 6.88(\mathrm{t}, J=7.6 \mathrm{~Hz}, 2 \mathrm{H}), 6.77$ $(\mathrm{t}, J=7.3 \mathrm{~Hz}, 1 \mathrm{H}), 5.42(\mathrm{~s}, 1 \mathrm{H}), 5.27(\mathrm{~s}, 1 \mathrm{H}), 5.05(\mathrm{dd}, J=8.4,2.7 \mathrm{~Hz}, 1 \mathrm{H}), 4.85(\mathrm{dd}, J=15.8$, $11.5 \mathrm{~Hz}, 3 \mathrm{H}), 4.76-4.47(\mathrm{~m}, 7 \mathrm{H}), 4.37(\mathrm{dd}, J=10.7,8.7 \mathrm{~Hz}, 1 \mathrm{H}), 4.28(\mathrm{~d}, J=12.2 \mathrm{~Hz}, 1 \mathrm{H})$, $4.20-3.98(\mathrm{~m}, 3 \mathrm{H}), 3.95-3.85(\mathrm{~m}, 4 \mathrm{H}), 3.79-3.70(\mathrm{~m}, 5 \mathrm{H}), 3.63(\mathrm{~d}, J=25.1 \mathrm{~Hz}, 4 \mathrm{H}), 3.32$ $(\mathrm{dt}, J=9.7,6.8 \mathrm{~Hz}, 1 \mathrm{H}), 2.15(\mathrm{t}, J=7.2 \mathrm{~Hz}, 2 \mathrm{H}), 1.97(\mathrm{~s}, 3 \mathrm{H}), 1.42-1.25(\mathrm{~m}, 3 \mathrm{H}), 1.17(\mathrm{dt}, J$ $=15.0,6.9 \mathrm{~Hz}, 3 \mathrm{H}), 1.13-1.03(\mathrm{~m}, 7 \mathrm{H}), 1.04(\mathrm{~s}, 22 \mathrm{H}) .{ }^{13} \mathrm{C} \mathrm{NMR}\left(101 \mathrm{MHz}, \mathrm{CDCl}_{3}\right) \delta 170.0$, $138.7,138.2$, 138.0, 137.8, 133.7, 128.7, 128.4, 128.34, 128.3, 128.2, 128.0, 128.0, 127.9, $127.9,127.9,127.6,127.6,127.3,127.29,127.07,126.83,99.1,98.0,81.0,77.9,76.8,75.2$, 74.6, 74.5, 73.7, 73.5, 73.2, 71.8, 69.3, 69.2, 68.9, 62.1, 55.6, 36.1, 31.0, 29.0, 28.9, 28.3, 26.8, 25.3, 20.8, 18.0, 17.9, 12.0, 11.9. ESI-MS $(\mathrm{m} / \mathrm{z}):[\mathrm{M}+\mathrm{Na}]^{+}$cald 1258.58 obsd 1528.4

2-O-Acetyl-3,4-di-O-benzyl- $\alpha$-D-mannopyranosyl-(1 $\rightarrow 4)-1-O-(6-t h i o b e n z y l) h e x y l-3,6-O-$ benzyl-2-deoxy-2-N-phthalimido- $\beta$-D-glucopyranoside (10)

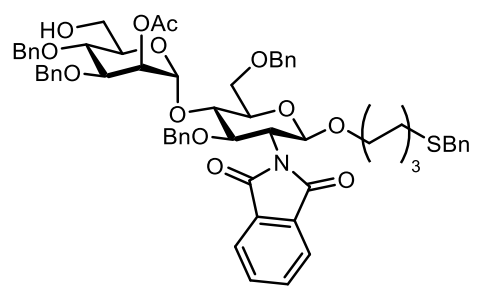

To a stirred solution of disaccharide 9 ( $0.16 \mathrm{~g}, 0.13 \mathrm{mmol})$ in THF (4 mL) was added $70 \% \mathrm{HF}$ in pyridine $(0.08 \mathrm{~mL}, 0.65 \mathrm{mmol})$. The reaction mixture was stirred for $48 \mathrm{~h}$ at room temperature. The reaction was quenched with aq. $\mathrm{NaHCO}_{3}$, extracted with $\mathrm{CH}_{2} \mathrm{Cl}_{2}$, washed with brine and dried over $\mathrm{Na}_{2} \mathrm{SO}_{4}$. The solvents were removed under reduced pressure and the crude product was purified by flash column chromatography to obtain the disaccharide acceptor $\mathbf{1 0}$ (0.12 g, $0.11 \mathrm{mmol}, 83 \%) . \mathrm{R}_{f}=0.4($ Hexanes/EtOAc $=2: 1){ }^{1} \mathrm{H} \mathrm{NMR}\left(400 \mathrm{MHz}, \mathrm{CDCl}_{3}\right) \delta 7.71$ $-7.63(\mathrm{~m}, 6 \mathrm{H}), 7.51-7.21(\mathrm{~m}, 20 \mathrm{H}), 7.07-6.91(\mathrm{~m}, 4 \mathrm{H}), 6.88-6.79(\mathrm{~m}, 1 \mathrm{H}), 5.55-5.47(\mathrm{~m}$, $1 \mathrm{H}), 5.35(\mathrm{~d}, J=1.4 \mathrm{~Hz}, 1 \mathrm{H}), 5.07(\mathrm{~d}, J=8.5 \mathrm{~Hz}, 1 \mathrm{H}), 4.88(\mathrm{dd}, J=24.8,11.5 \mathrm{~Hz}, 1 \mathrm{H}), 4.78-$ $4.60(\mathrm{~m}, 3 \mathrm{H}), 4.63-4.51(\mathrm{~m}, 1 \mathrm{H}), 4.44(\mathrm{ddd}, J=11.4,8.7,2.9 \mathrm{~Hz}, 1 \mathrm{H}), 4.32(\mathrm{~d}, J=12.2 \mathrm{~Hz}$, $1 \mathrm{H}), 4.24-4.11(\mathrm{~m}, 1 \mathrm{H}), 4.10-3.89(\mathrm{~m}, 2 \mathrm{H}), 3.90-3.58(\mathrm{~m}, 10 \mathrm{H}), 3.36(\mathrm{dt}, J=9.8,6.6 \mathrm{~Hz}$, $1 \mathrm{H}), 2.19(\mathrm{t}, J=7.3 \mathrm{~Hz}, 1 \mathrm{H}), 2.05(\mathrm{~s}, 3 \mathrm{H}), 1.38(\mathrm{ddt}, J=30.1,13.0,6.8 \mathrm{~Hz}, 3 \mathrm{H}), 1.30-0.84$ $(\mathrm{m}, 7 \mathrm{H}) .{ }^{13} \mathrm{C} \mathrm{NMR}\left(101 \mathrm{MHz}, \mathrm{CDCl}_{3}\right) \delta 169.9,138.5,138.1,138.0,137.8,137.7,133.8,130.0$, $129.0,128.8,128.4,128.4,128.3,128.1,128.0,128.0,127.8,127.8,127.7,127.6,127.2$, 127.1, $126.9,99.2,98.0,81.2,77.9,76.9,75.3,74.9,74.70,74.0,73.6,73.1,71.8,69.4,68.8,68.6$, $62.0,55.8,36.2,31.0,29.1,28.9,28.4,25.4,20.9$. ESI-MS $(\mathrm{m} / \mathrm{z}):[\mathrm{M}+\mathrm{Na}]^{+}$cald 1102.43 obsd 1102.4 


\section{Synthesis of fragment 1}

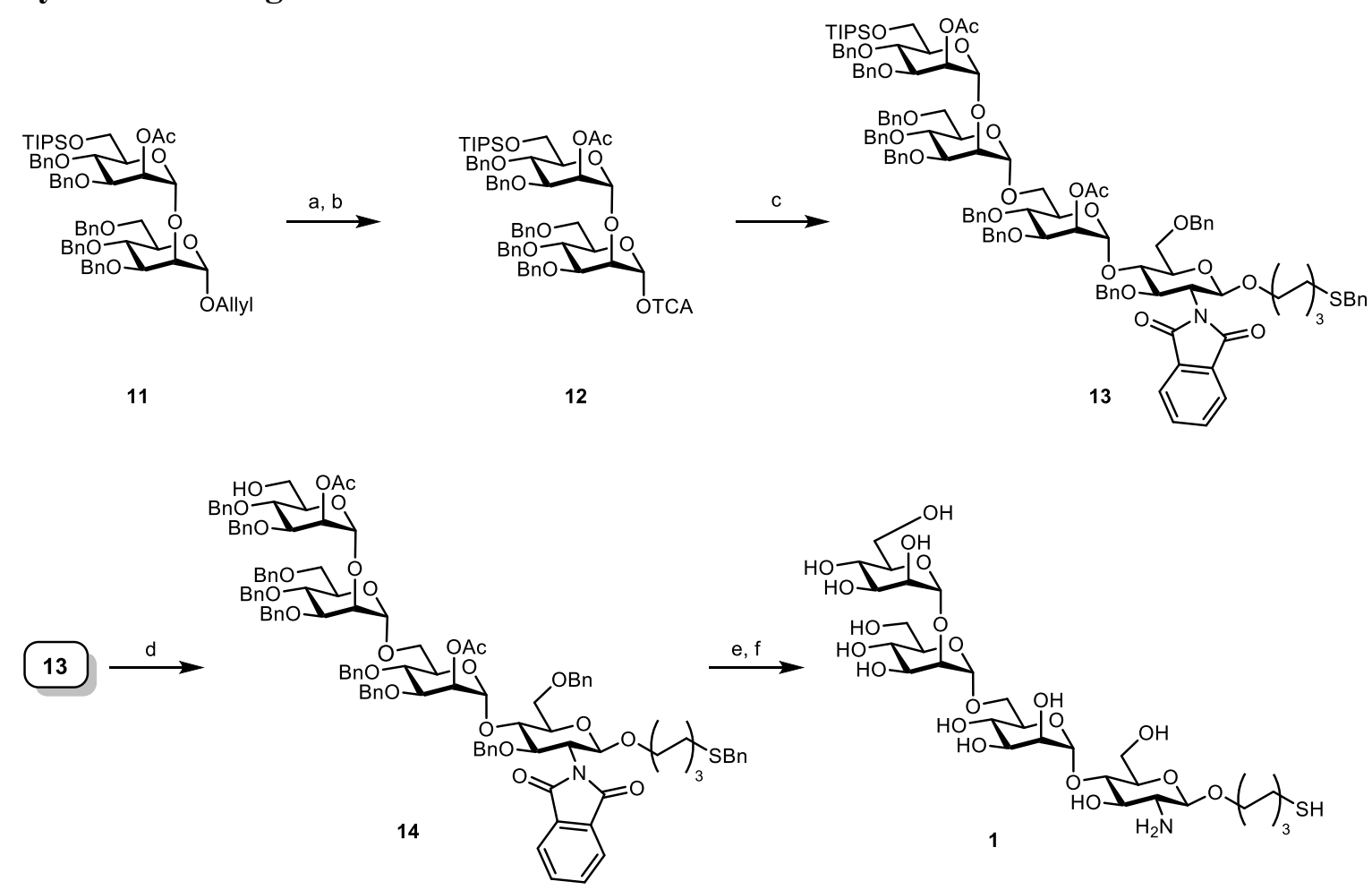

Scheme S2: Synthesis of fragment GPI 1. a) i. $\mathrm{H}_{2}$, $\left[\operatorname{Ir}(\mathrm{COD})\left(\mathrm{PMePh}_{2}\right)_{2}\right] \mathrm{PF}_{6}, \mathrm{THF}, \mathrm{rt}, 12 \mathrm{~h}$; ii. $\mathrm{HgO}$, $\mathrm{HgCl}_{2}$, acetone- $\mathrm{H}_{2} \mathrm{O}(5: 1)$, rt, $2 \mathrm{~h}, 81 \%$; b) $\mathrm{CCl}_{3} \mathrm{CN}, \mathrm{DBU}, \mathrm{CH}_{2} \mathrm{Cl}_{2}, 0{ }^{\circ} \mathrm{C}, 2 \mathrm{~h}, 86 \%$; c) TBSOTf, $\mathrm{Et}_{2} \mathrm{O}$, $0{ }^{\circ} \mathrm{C}, 2 \mathrm{~h}, 55 \%$ ( $\alpha$-isomer); d) $\mathrm{Sc}(\mathrm{OTf})_{3}, \mathrm{H}_{2} \mathrm{O}, \mathrm{CH}_{3} \mathrm{CN}, 50{ }^{\circ} \mathrm{C}, 12 \mathrm{~h}, 71 \%$; e) $\left(\mathrm{CH}_{2} \mathrm{NH}_{2}\right)_{2}, \mathrm{H}_{2} \mathrm{O}, n-\mathrm{BuOH}$, $90^{\circ} \mathrm{C}, 4$ h; f) i. $\mathrm{Na}$, liq. $\mathrm{NH}_{3}$, THF, $-78^{\circ} \mathrm{C}, 1 \mathrm{~h}$; ii. $\mathrm{MeOH}, \mathrm{rt}, 1 \mathrm{~h}, 57 \%$ (over two steps).

2-O-Acetyl-3,4-di-O-benzyl-6-O-triisopropylsilyl- $\alpha$-D-mannopyranosyl-(1 $\rightarrow 2)-3,4,6$-tri- $\quad O$ benzyl- $\alpha-D$-mannopyranosyl trichloroacetimidate (12)

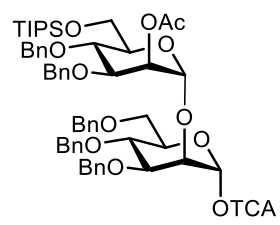

A solution of $\left[\operatorname{IrCOD}\left(\mathrm{PPh}_{2} \mathrm{Me}\right)_{2}\right] \mathrm{PF}_{6}(5.0 \mathrm{mg}, 5.8 \mu \mathrm{mol})$ in $\mathrm{THF}(3 \mathrm{~mL})$ was stirred under hydrogen atmosphere until the color turned from red to colorless to pale yellow. The hydrogen atmosphere was exchanged with Argon. The activated catalyst solution was added to a solution of disaccharide $11(0.30 \mathrm{~g}, 0.29 \mathrm{mmol})$ in THF $(10 \mathrm{~mL})$ and stirred at room temperature. After $16 \mathrm{~h}$, the solvent was removed and the residue was dissolved in a mixture of acetone $(5.2 \mathrm{~mL})$ and water $(0.6 \mathrm{~mL})$. Mercury (II) chloride $(0.39 \mathrm{~g}, 1.45 \mathrm{mmol})$ and mercury (II) oxide (0.01 $\mathrm{mg}, 0.044 \mathrm{mmol}$ ) were added. After $1 \mathrm{~h}$, saturated aq. $\mathrm{NaHCO}_{3}$ was added to reaction mixture and was extracted three times with $\mathrm{CH}_{2} \mathrm{Cl}_{2}$. The combined organic layers were dried over $\mathrm{Na}_{2} \mathrm{SO}_{4}$ and concentrated. The crude product was purified by flash column chromatography to obtain disaccharide hemiacetal intermediate $(0.24 \mathrm{~g}, 0.24 \mathrm{mmol}, 81 \%)$ as colorless oil.

To a stirred solution of hemiacetal $(0.285 \mathrm{~g}, 0.28 \mathrm{mmol})$ in $\mathrm{CH}_{2} \mathrm{Cl}_{2}(13 \mathrm{~mL})$ at $0{ }^{\circ} \mathrm{C}$ were added $\mathrm{CCl}_{3} \mathrm{CN}(0.29 \mathrm{~mL}, 2.88 \mathrm{mmol})$ and $\mathrm{DBU}(0.009 \mathrm{~mL}, 0.06 \mathrm{mmol})$. The reaction mixture was stirred for $1 \mathrm{~h}$ at $0{ }^{\circ} \mathrm{C}$. The resulting mixture was concentrated and purified by flash column 
chromatography to obtain imidate donor $12(0.28 \mathrm{~g}, 0.25 \mathrm{mmol}, 86 \%)$ as clear oil. $\mathrm{R}_{f}=0.4$ $($ EtOAc/hexane $=2: 3){ }^{1} \mathrm{H} \mathrm{NMR}\left(400 \mathrm{MHz}, \mathrm{CDCl}_{3}\right) \delta 8.53(\mathrm{~s}, 1 \mathrm{H}), 7.39-7.31(\mathrm{~m}, 5 \mathrm{H}), 7.31-$ $7.13(\mathrm{~m}, 17 \mathrm{H}), 6.29(\mathrm{~s}, 1 \mathrm{H}), 5.51(\mathrm{~s}, 1 \mathrm{H}), 5.15(\mathrm{~s}, 1 \mathrm{H}), 4.93-4.85(\mathrm{~m}, 2 \mathrm{H}), 4.79-4.68(\mathrm{~m}$, $3 \mathrm{H}), 4.67-4.56(\mathrm{~m}, 2 \mathrm{H}), 4.47(\mathrm{dd}, J=28.1,11.5 \mathrm{~Hz}, 2 \mathrm{H}), 4.12(\mathrm{~s}, 1 \mathrm{H}), 4.08-3.91(\mathrm{~m}, 8 \mathrm{H})$, $3.86-3.77(\mathrm{~m}, 2 \mathrm{H}), 3.70(\mathrm{~d}, J=11.3 \mathrm{~Hz}, 1 \mathrm{H}), 2.11(\mathrm{~s}, 3 \mathrm{H}), 1.08(\mathrm{~d}, J=4.7 \mathrm{~Hz}, 21 \mathrm{H})$.

1-O-(6-thiobenzyl)hexyl-2-O-acetyl-3,4-di-O-benzyl-6-O-triisopropylsilyl- $\alpha$-Dmannopyranosyl-( $(1 \rightarrow 2)$-3,4,6-tri-O-benzyl- $\alpha$-D-mannopyranosyl-( $1 \rightarrow 6)$-2-O-Acetyl-3,4-diO-benzyl- $\alpha$-D-mannopyranosyl-( $1 \rightarrow 4)$-3, 6-O-benzyl-2-deoxy-2-N-phthalimido- $\beta$ - $D$ glucopyranoside (13)

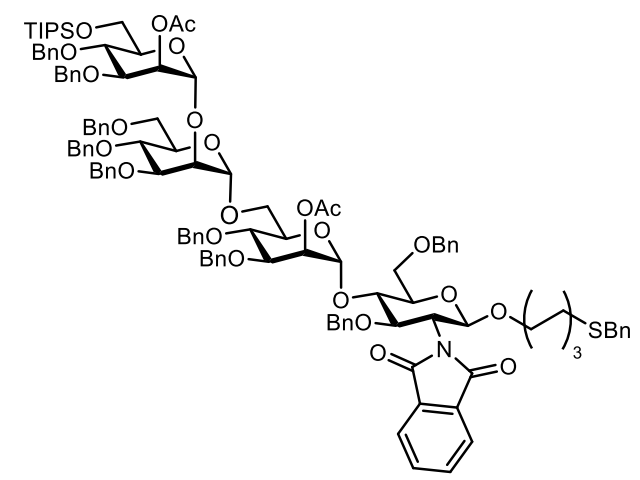

The disaccharide imidate $12(0.18 \mathrm{~g}, 0.16 \mathrm{mmol})$ and disaccharide acceptor $9(0.14 \mathrm{~g}, 0.13$ mmol) were co-evaporated with toluene $(3 \times 5 \mathrm{~mL})$ and dried under high vacuum for $2 \mathrm{~h}$. The mixture was dissolved in a mixture of anhydrous diethylether and anhydrous $\mathrm{CH}_{2} \mathrm{Cl}_{2}(1: 1,6$ $\mathrm{mL}$ ) and activated molecular sieves were added. The solution was stirred for $10 \mathrm{~min}$ at room temperature and cooled to $0{ }^{\circ} \mathrm{C}$. The mixture was treated with TBSOTf $(9 \mu \mathrm{L}, 0.04 \mathrm{mmol})$ and stirred at $0{ }^{\circ} \mathrm{C}$ for a period of $1 \mathrm{~h}$. The reaction was diluted with $\mathrm{CH}_{2} \mathrm{Cl}_{2}$, quenched with $\mathrm{Et}_{3} \mathrm{~N}$ and concentrated. The crude product was purified by flash column chromatography to obtain tetrasaccharide $13(0.15 \mathrm{~g}, 0.07 \mathrm{mmol}, 55 \% \alpha$-isomer $) . \mathrm{R}_{f}=0.3($ EtOAc/hexane $=2: 3){ }^{1} \mathrm{H}$ NMR (400 MHz, $\left.\mathrm{CDCl}_{3}\right) \delta 7.68-7.59(\mathrm{~m}, 6 \mathrm{H}), 7.31(\mathrm{dd}, J=17.9,11.8 \mathrm{~Hz}, 30 \mathrm{H}), 7.21(\mathrm{dd}, J$ $=14.7,7.7 \mathrm{~Hz}, 36 \mathrm{H}), 7.17-7.08(\mathrm{~m}, 7 \mathrm{H}), 7.01(\mathrm{~d}, J=7.4 \mathrm{~Hz}, 3 \mathrm{H}), 6.90(\mathrm{t}, J=7.5 \mathrm{~Hz}, 3 \mathrm{H})$, $6.79(\mathrm{t}, J=7.2 \mathrm{~Hz}, 1 \mathrm{H}), 5.49(\mathrm{~d}, J=13.5 \mathrm{~Hz}, 3 \mathrm{H}), 5.34(\mathrm{~s}, 1 \mathrm{H}), 5.15(\mathrm{~s}, 1 \mathrm{H}), 5.08-5.01(\mathrm{~m}$, $2 \mathrm{H}), 4.93-4.79(\mathrm{~m}, 8 \mathrm{H}), 4.72-4.27(\mathrm{~m}, 23 \mathrm{H}), 4.23-3.99(\mathrm{~m}, 7 \mathrm{H}), 4.02-3.79(\mathrm{~m}, 16 \mathrm{H}), 3.77$ $(\mathrm{t}, J=10.2 \mathrm{~Hz}, 7 \mathrm{H}), 3.75-3.58(\mathrm{~m}, 7 \mathrm{H}), 3.55-3.38(\mathrm{~m}, 5 \mathrm{H}), 3.39-3.21(\mathrm{~m}, 3 \mathrm{H}), 2.41-2.28$ (m, 2H), 2.15 (t, $J=7.2 \mathrm{~Hz}, 1 \mathrm{H}), 2.07$ (s, 3H), 1.98 (s, 3H), 1.08 (d, $J=4.1 \mathrm{~Hz}, 30 \mathrm{H}), 0.96$ (d, $J=36.0 \mathrm{~Hz}, 2 \mathrm{H}), 0.92-0.81(\mathrm{~m}, 23 \mathrm{H}) .{ }^{13} \mathrm{C} \mathrm{NMR}\left(101 \mathrm{MHz}, \mathrm{CDCl}_{3}\right) \delta 170.1,169.8,138.8$, 138.5, 138.4, 138.1, 138.0, 138.0, 137.7, 137.6, 133.7, 129.9, 128.9, 128.7, 128.4, 128.3, 128.3, $128.3,128.2$, 128.2, 128.1, 128.1, 127.9, 127.9, 127.9, 127.7, 127.5, 127.49, 127.4, 127.3, $127.2,127.2,127.1,126.85,99.3,99.0,98.8,98.0,80.9,79.6,78.4,77.9,75.2,75.0,74.7,74.5$, $74.2,73.77,73.3,73.1,71.88,71.8,71.7,71.5,69.3,68.8,68.5,66.1,62.5,55.7,36.2,32.3$, $31.9,31.0,29.7,29.37,29.0,28.9,28.3,26.3,25.3,22.7,21.0,20.95,18.1,18.0,14.1,12.0$. HRMS $(\mathrm{m} / \mathrm{z}):[\mathrm{M}+\mathrm{H}]^{+}$cald 2053.0140 obsd 2053.0144

1-O-(6-thiobenzyl)hexyl-2-O-acetyl-3,4-di-O-benzyl- $\alpha$-D-mannopyranosyl-(1 $\rightarrow 2)-3,4,6$-tri$O$-benzyl- $\alpha$-D-mannopyranosyl-( $1 \rightarrow 6)-2-O$-Acetyl-3,4-di-O-benzyl- $\alpha$-D-mannopyranosyl(1 $\rightarrow 4$ )-3,6-O-benzyl-2-deoxy-2-N-phthalimido- $\beta$-D-glucopyranoside (14) 


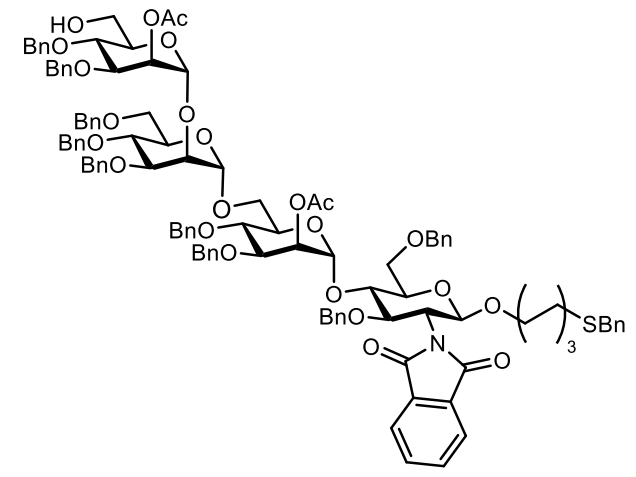

To a stirred solution of tetrasaccharide $13(0.13 \mathrm{~g}, 0.190 \mathrm{mmol})$ in $\mathrm{CH}_{3} \mathrm{CN}(7 \mathrm{~mL})$ were added water $(50 \mu \mathrm{L})$ and $\mathrm{Sc}(\mathrm{OTf})_{3}(0.093 \mathrm{~g}, 0.19 \mathrm{mmol})$. The reaction mixture was heated up to 50 ${ }^{\circ} \mathrm{C}$ ans stirred for $6 \mathrm{~h}$. The reaction was quenched with $\mathrm{Et}_{3} \mathrm{~N}$ and concentrated. The crude product was purified by flash column chromatography to obtain $14(0.09 \mathrm{~g}, 0.05 \mathrm{mmol}, 71 \%)$. $\mathrm{R}_{f}=0.35($ EtOAc/hexane $=1: 1){ }^{1} \mathrm{H}$ NMR $\left(400 \mathrm{MHz}, \mathrm{CDCl}_{3}\right) \delta 7.63(\mathrm{~d}, J=16.4 \mathrm{~Hz}, 4 \mathrm{H}), 7.30$ $(\mathrm{dd}, J=9.4,6.1 \mathrm{~Hz}, 17 \mathrm{H}), 7.23(\mathrm{~d}, J=7.3 \mathrm{~Hz}, 22 \mathrm{H}), 7.16(\mathrm{dd}, J=14.8,5.9 \mathrm{~Hz}, 9 \mathrm{H}), 7.00(\mathrm{~d}, J$ $=7.5 \mathrm{~Hz}, 2 \mathrm{H}), 6.89(\mathrm{t}, J=7.5 \mathrm{~Hz}, 2 \mathrm{H}), 6.77(\mathrm{t}, J=7.3 \mathrm{~Hz}, 1 \mathrm{H}), 5.48(\mathrm{~s}, 2 \mathrm{H}), 5.32(\mathrm{~s}, 1 \mathrm{H}), 5.07$ - $4.97(\mathrm{~m}, 2 \mathrm{H}), 4.95-4.83(\mathrm{~m}, 3 \mathrm{H}), 4.82(\mathrm{~d}, J=11.5 \mathrm{~Hz}, 3 \mathrm{H}), 4.69-4.54(\mathrm{~m}, 8 \mathrm{H}), 4.50$ (dd, $J=20.1,9.3 \mathrm{~Hz}, 3 \mathrm{H}), 4.47-4.33(\mathrm{~m}, 5 \mathrm{H}), 4.29(\mathrm{~d}, J=12.2 \mathrm{~Hz}, 1 \mathrm{H}), 4.22-4.13(\mathrm{~m}, 1 \mathrm{H}), 4.07$ (s, 1H), $4.01-3.68(\mathrm{~m}, 24 \mathrm{H}), 3.65-3.53(\mathrm{~m}, 5 \mathrm{H}), 3.53-3.44(\mathrm{~m}, 3 \mathrm{H}), 3.37-3.21(\mathrm{~m}, 2 \mathrm{H})$, $2.14(\mathrm{t}, J=6.7 \mathrm{~Hz}, 2 \mathrm{H}), 2.08(\mathrm{~s}, 3 \mathrm{H}), 1.98(\mathrm{~s}, 3 \mathrm{H}), 1.37(\mathrm{dd}, J=15.8,8.1 \mathrm{~Hz}, 3 \mathrm{H}), 1.35-1.22$ $(\mathrm{m}, 3 \mathrm{H}), 1.15(\mathrm{dd}, J=15.2,7.9 \mathrm{~Hz}, 3 \mathrm{H}), 1.12-0.91(\mathrm{~m}, 6 \mathrm{H}), 0.86(\mathrm{~d}, J=10.4 \mathrm{~Hz}, 3 \mathrm{H})$. . ESIMS $(m / z):[\mathrm{M}+\mathrm{Na}]^{+}$cald 1918.80 obsd 1918.5

1-O-(6-thio)hexyl- $\alpha$-D-mannopyranosyl- $(1 \rightarrow 2)$ - $\alpha$-D-mannopyranosyl- $(1 \rightarrow 6)-\alpha$-Dmannopyranosyl-(1 $\rightarrow 4)$-2-amino-2-deoxy- $\beta$-D-glucopyranoside $\mathbf{( 1 )}$

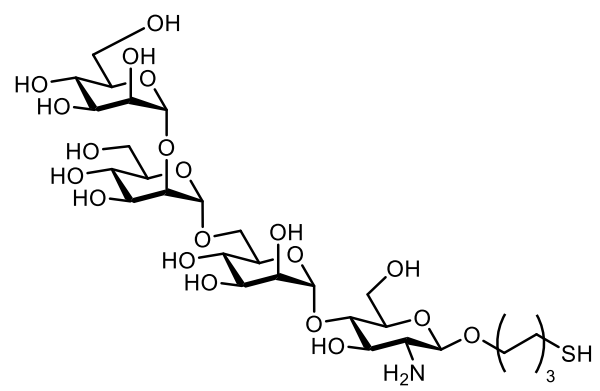

Tetrasaccharide $14(0.03 \mathrm{~g}, 0.02 \mathrm{mmol})$ was dissolved in a mixture of ethylenediamine $(2 \mathrm{~mL})$ and butanol $(2 \mathrm{~mL})$ and stirred at $90{ }^{\circ} \mathrm{C}$ for $3 \mathrm{~h}$. After $3 \mathrm{~h}$, the reaction mixture was concentrated to obtain partially deprotected crude intermediate. The crude intermediate was dissolved in anhydrous THF and $\mathrm{MeOH}$. This solution was added dropwise to blue solution of $20 \mathrm{~mL}$ liquefied ammonia with sodium $-78{ }^{\circ} \mathrm{C}$. The reaction was stirred at $-78{ }^{\circ} \mathrm{C}$. After $1 \mathrm{~h}$, the reaction was quenched with $\mathrm{MeOH}$ and stirred for additional $1 \mathrm{~h}$ at rt. Sodium methoxide generated in the reaction was quenched by dropwise addition of glacial acetic acid. The reaction mixture was concentrated and the crude product was purified by size exclusion column chromatography using a Sephadex ${ }^{\circledR}$ super fine G-15 (GE Healthcare) column and 5\% ethanol in water as eluent to obtain $1(7 \mathrm{mg}, 0.009 \mathrm{mmol}, 57 \%)$ as white solid. ${ }^{1} \mathrm{H}$ NMR $(600 \mathrm{MHz}$, Deuterium Oxide) $\delta 5.08(\mathrm{~s}, 1 \mathrm{H}), 5.02(\mathrm{~s}, 1 \mathrm{H}), 4.91(\mathrm{~s}, 1 \mathrm{H}), 4.53-4.37(\mathrm{~m}, 0 \mathrm{H}), 3.94(\mathrm{ddd}, J$ $=5.6,3.1,1.9 \mathrm{~Hz}, 3 \mathrm{H}), 3.91-3.88(\mathrm{~m}, 2 \mathrm{H}), 3.85-3.74(\mathrm{~m}, 9 \mathrm{H}), 3.75-3.52(\mathrm{~m}, 22 \mathrm{H}), 3.53-$ $3.42(\mathrm{~m}, 4 \mathrm{H}), 3.23-3.08(\mathrm{~m}, 1 \mathrm{H}), 2.97-2.91(\mathrm{~m}, 1 \mathrm{H}), 2.86-2.72(\mathrm{~m}, 1 \mathrm{H}), 2.64(\mathrm{t}, J=7.2$ $\mathrm{Hz}, 1 \mathrm{H}), 1.55$ (ddd, $J=25.0,14.1,7.0 \mathrm{~Hz}, 6 \mathrm{H}), 1.30-1.25(\mathrm{~m}, 6 \mathrm{H}) .{ }^{13} \mathrm{C}$ NMR $\left(151 \mathrm{MHz}, \mathrm{d}_{2} \mathrm{O}\right)$ 
$\delta 104.9,104.5,100.9,81.2,79.7,75.8,75.3,74.8,72.9,72.7,72.5,69.5,69.4,69.0,63.7,63.5$, $30.7,29.8,27.2,25.8$. HRMS $(\mathrm{m} / \mathrm{z})$ of the oxidized compound: $[\mathrm{M}+2 \mathrm{H}]^{2+}$ cald 781.3033 obsd 781.3027

\section{Synthesis of fragment 2}

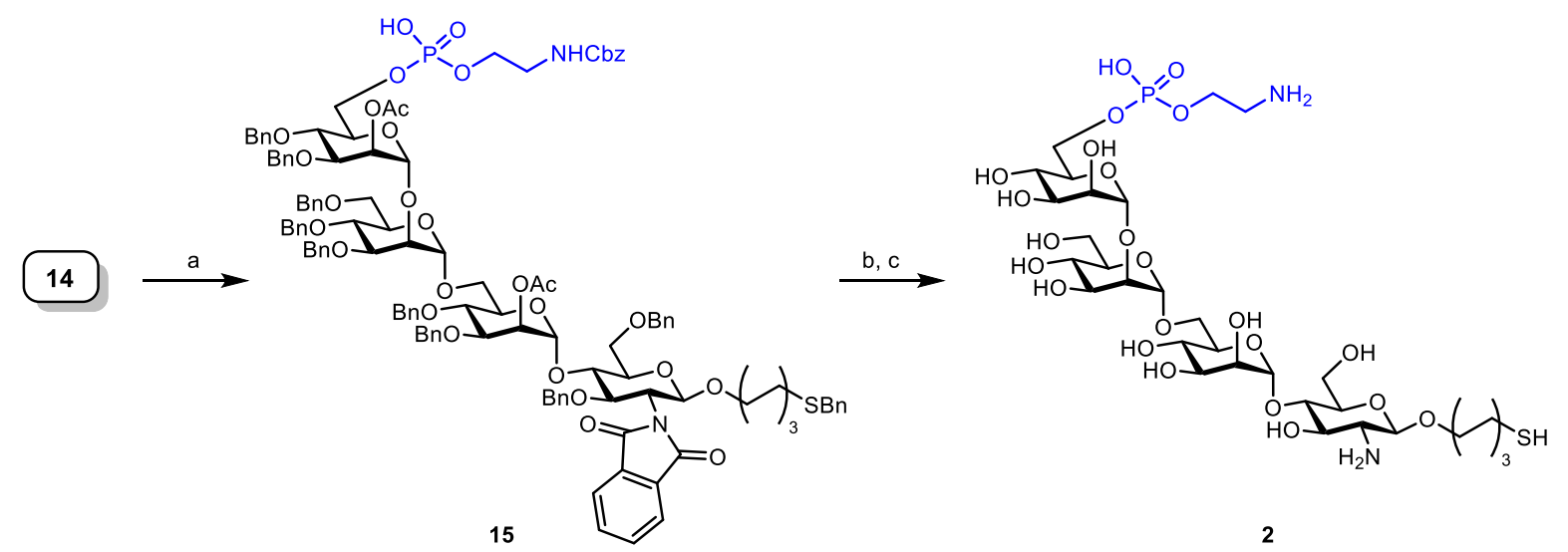

Scheme S3: Synthesis of fragment GPI 2. a) i. 2-3, PivCl, py, rt, 16 h; ii. $\mathrm{I}_{2}, \mathrm{H}_{2} \mathrm{O}$, py, rt, $1 \mathrm{~h}, 66 \%$; b) $\left(\mathrm{CH}_{2} \mathrm{NH}_{2}\right)_{2}, \mathrm{H}_{2} \mathrm{O}, n-\mathrm{BuOH}, 90^{\circ} \mathrm{C}, 4 \mathrm{~h}$; c) i. Na, liq. $\mathrm{NH}_{3}$, THF, $-78{ }^{\circ} \mathrm{C}, 1 \mathrm{~h}$; ii. $\mathrm{MeOH}, \mathrm{rt}, 1 \mathrm{~h}, 75 \%$ (over two steps).

1-O-(6-thiobenzyl)hexyl-2-O-Acetyl-3,4-di-O-benzyl-6-O-(2-N-benzyloxycarbonyl) aminoethyl-phosphonato- $\alpha$-D-mannopyranosyl-( $1 \rightarrow 2)$-3,4,6-tri-O-benzyl- $\alpha$ - $D$ -

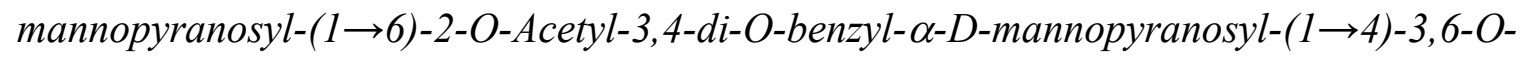
benzyl-2-deoxy-2-N-phthalimido- $\beta$-D-glucopyranoside (15)

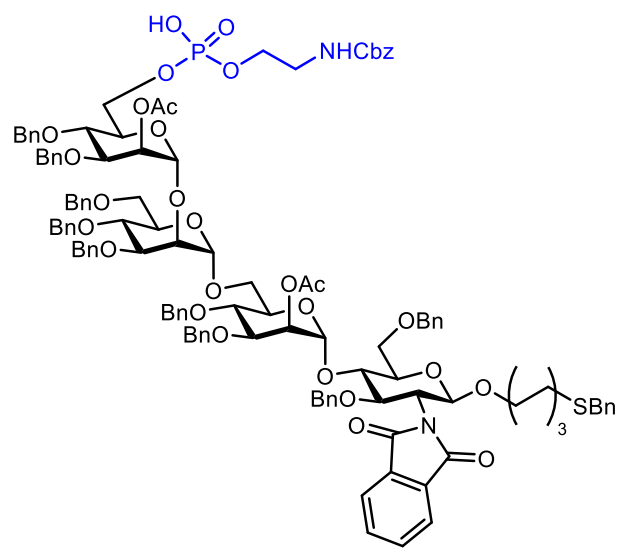

Tetrasaccharide $14(0.02 \mathrm{~g}, 10.5 \mu \mathrm{mol})$ and $H$-phosphonatethanolamine $(0.01 \mathrm{~g}, 0.03 \mathrm{mmol})$ were co-evaporated with pyridine for three times and dried under high vacuum for $2 \mathrm{~h}$. The mixture was dissolved in anhydrous pyridine $(5 \mathrm{~mL})$ and a solution of pivolyl chloride $(4 \mu \mathrm{L}$, $0.03 \mathrm{mmol})$ in pyridine $(1 \mathrm{~mL})$ was added. The solution was stirred for $6 \mathrm{~h}$ at room temperature. After $6 \mathrm{~h}$, iodine $(8 \mathrm{mg}, 0.03 \mathrm{mmol})$ and water $(0.05 \mathrm{~mL})$ were added and reaction was stirred for additional $2 \mathrm{~h}$ at room temperature. The reaction mixture was quenched with $\mathrm{Na}_{2} \mathrm{~S}_{2} \mathrm{O}_{3}$ and extracted with $\mathrm{CH}_{2} \mathrm{Cl}_{2}$. The organic layer were concentrated and purified by $\mathrm{Et}_{3} \mathrm{~N}$ deactivated silica gel flash column chromatography to obtain phosphorylated tetrasaccharide $\mathbf{1 5}(15 \mathrm{mg}, 7$ $\mu \mathrm{mol}, 66 \%) . \mathrm{R}_{f}=0.5\left(\mathrm{MeOH} / \mathrm{CH}_{2} \mathrm{Cl}_{2}=1: 10\right){ }^{1} \mathrm{H} \mathrm{NMR}\left(400 \mathrm{MHz}, \mathrm{CDCl}_{3}\right) \delta 7.64(\mathrm{~d}, J=16.6$ $\mathrm{Hz}, 4 \mathrm{H}), 7.21(\mathrm{dd}, J=11.7,8.2 \mathrm{~Hz}, 27 \mathrm{H}), 7.15(\mathrm{dd}, J=10.7,5.4 \mathrm{~Hz}, 15 \mathrm{H}), 7.14-7.01(\mathrm{~m}$, 
4H), $5.48(\mathrm{~d}, J=8.0 \mathrm{~Hz}, 2 \mathrm{H}), 5.37(\mathrm{~s}, 1 \mathrm{H}), 5.25(\mathrm{~s}, 0 \mathrm{H}), 5.09(\mathrm{~s}, 1 \mathrm{H}), 5.03-4.93(\mathrm{~m}, 1 \mathrm{H}), 4.87$ $-4.67(\mathrm{~m}, 5 \mathrm{H}), 4.64-4.34(\mathrm{~m}, 9 \mathrm{H}), 3.96-3.85(\mathrm{~m}, 5 \mathrm{H}), 3.76(\mathrm{td}, J=23.3,21.0,9.8 \mathrm{~Hz}, 7 \mathrm{H})$, $3.65-3.52(\mathrm{~m}, 4 \mathrm{H}), 3.37-3.17(\mathrm{~m}, 7 \mathrm{H}), 2.11(\mathrm{t}, J=7.2 \mathrm{~Hz}, 1 \mathrm{H}), 2.00-1.90(\mathrm{~m}, 6 \mathrm{H}), 1.53(\mathrm{t}$, $J=7.3 \mathrm{~Hz}, 0 \mathrm{H}), 1.25-1.16(\mathrm{~m}, 4 \mathrm{H}), 1.14-0.94(\mathrm{~m}, 3 \mathrm{H}) .{ }^{31} \mathrm{P}$ NMR $\left(162 \mathrm{MHz}, \mathrm{CDCl}_{3}\right) \delta-$ 1.95. ESI-MS $(\mathrm{m} / \mathrm{z}):[\mathrm{M}+\mathrm{Na}]^{+}$cald 2176.83 obsd 2176.9

1-O-(6-thio)hexyl-6-O-aminoethyl-phosphonato- $\alpha$-D-mannopyranosyl- $(1 \rightarrow 2)-\alpha-D$ mannopyranosyl-( $1 \rightarrow 6)$ - $\alpha$-D-mannopyranosyl-( $1 \rightarrow 4)$-2-deoxy-2-amino- $\beta$ - $D$ glucopyranoside (2)

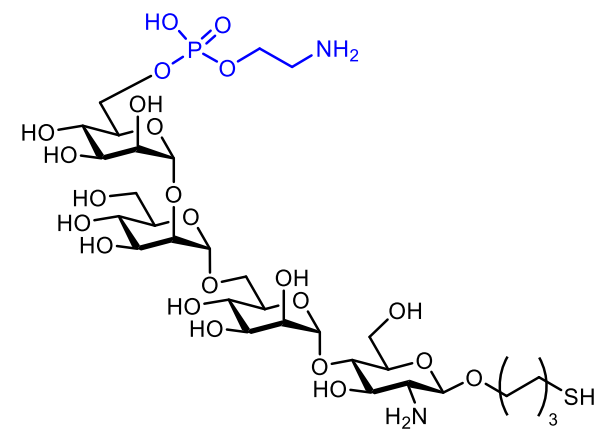

Tetrasaccharide $15(16 \mathrm{mg}, 7.5 \mu \mathrm{mol})$ was dissolved in a mixture of ethylenediamine $(2 \mathrm{~mL})$ and butanol $(2 \mathrm{~mL})$ and stirred at $90^{\circ} \mathrm{C}$ for $3 \mathrm{~h}$. After $3 \mathrm{~h}$, the reaction mixture was concentrated to obtain partially deprotected crude intermediate. The crude intermediate was dissolved in anhydrous THF and $\mathrm{MeOH}$. This solution was added dropwise to blue solution of $20 \mathrm{~mL}$ liquefied ammonia with sodium $-78{ }^{\circ} \mathrm{C}$. The reaction was stirred at $-78{ }^{\circ} \mathrm{C}$. After $1 \mathrm{~h}$, the reaction was quenched with $\mathrm{MeOH}$ and stirred for additional $1 \mathrm{~h}$ at $\mathrm{rt}$. Sodium methoxide generated in the reaction was quenched by dropwise addition of glacial acetic acid. The reaction mixture was concentrated and the crude product was purified by size exclusion column chromatography using a Sephadex ${ }^{\circledR}$ super fine G-15 (GE Healthcare) column and 5\% ethanol in water as eluent to obtain $2(5 \mathrm{mg}, 5.6 \mu \mathrm{mol}, 75 \%)$ as white solid. ${ }^{1} \mathrm{H}$ NMR $\left(600 \mathrm{MHz}, \mathrm{D}_{2} \mathrm{O}\right)$ $\delta 5.14-5.06(\mathrm{~m}, 1 \mathrm{H}), 5.04-4.96(\mathrm{~m}, 1 \mathrm{H}), 4.93-4.87(\mathrm{~m}, 1 \mathrm{H}), 4.37(\mathrm{~d}, J=8.2 \mathrm{~Hz}, 1 \mathrm{H}), 4.06$ $-3.39(\mathrm{~m}, 26 \mathrm{H}), 3.21-3.13(\mathrm{~m}, 2 \mathrm{H}), 2.83-2.74(\mathrm{~m}, 1 \mathrm{H}), 2.67(\mathrm{~s}, 1 \mathrm{H}), 1.62(\mathrm{dt}, J=15.6,7.5$ $\mathrm{Hz}, 2 \mathrm{H}), 1.54-1.47(\mathrm{~m}, 2 \mathrm{H}), 1.30$ (ddd, $J=23.9,13.9,6.8 \mathrm{~Hz}, 4 \mathrm{H}) .{ }^{13} \mathrm{C}$ NMR $\left(151 \mathrm{MHz}, \mathrm{D}_{2} \mathrm{O}\right)$ $\delta 105.0,104.4,100.8,81.5,79.8,77.4,75.3,74.8,72.7,72.4,69.4,68.8,64.5,63.4,53.5,42.6$, 30.9, 29.9, 27.2, 26.4, 25.8. ${ }^{31} \mathrm{P}$ NMR $\left(243 \mathrm{MHz}, \mathrm{d}_{2} \mathrm{o}\right) \delta-2.75$. HRMS $(m / z):[\mathrm{M}+\mathrm{H}]^{+}$cald 905.2961 obsd 905.2836 


\section{Synthesis of fragment 3}

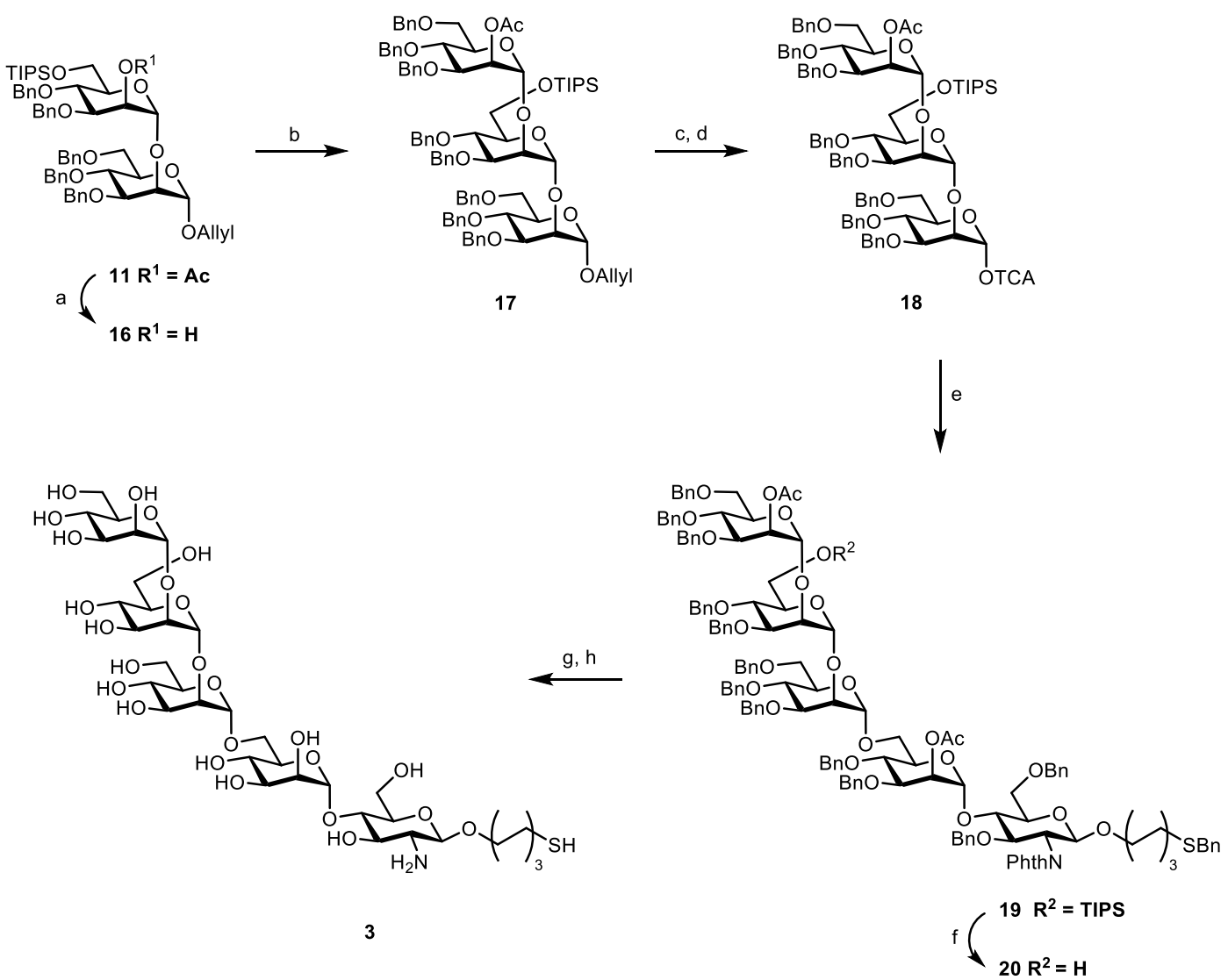

Scheme S4: Synthesis of fragment GPI 3. a) $\mathrm{NaOMe}, \mathrm{MeOH} / \mathrm{CH}_{2} \mathrm{Cl}_{2}, \mathrm{rt}, 12 \mathrm{~h}$, quant; b) TBSOTf, $\mathrm{Et}_{2} \mathrm{O}$, $0{ }^{\circ} \mathrm{C}, 2 \mathrm{~h}, 68 \%$; c) i. $\mathrm{H}_{2}$, [ $\left.\operatorname{Ir}(\mathrm{COD})\left(\mathrm{PMePh}_{2}\right)_{2}\right] \mathrm{PF}_{6}, \mathrm{THF}, \mathrm{rt}, 12 \mathrm{~h}$; ii. $\mathrm{HgO}, \mathrm{HgCl}_{2}$, acetone- $\mathrm{H}_{2} \mathrm{O}$ (5:1), rt, $2 \mathrm{~h}, 64 \%$; d) $\mathrm{CCl}_{3} \mathrm{CN}, \mathrm{DBU}, \mathrm{CH}_{2} \mathrm{Cl}_{2}, 0{ }^{\circ} \mathrm{C}, 2 \mathrm{~h}, 80 \%$; e) TBSOTf, $\mathrm{Et}_{2} \mathrm{O}, 0{ }^{\circ} \mathrm{C}, 2 \mathrm{~h}, 65 \%$ ( $\alpha$-isomer); f) $\mathrm{Sc}(\mathrm{OTf})_{3}, \mathrm{H}_{2} \mathrm{O}, \mathrm{CH}_{3} \mathrm{CN}, 50{ }^{\circ} \mathrm{C}, 12 \mathrm{~h}, 72 \%$; g) $\left(\mathrm{CH}_{2} \mathrm{NH}_{2}\right)_{2}, \mathrm{H}_{2} \mathrm{O}, n-\mathrm{BuOH}, 90{ }^{\circ} \mathrm{C}, 4 \mathrm{~h}$; h) i. Na, liq. $\mathrm{NH}_{3}$, THF, $-78^{\circ} \mathrm{C}, 1 \mathrm{~h}$; ii. $\mathrm{MeOH}, \mathrm{rt}, 1 \mathrm{~h}, 74 \%$ (over two steps).

n-Allyl-3,4-di-O-benzyl-6-O-triisopropylsilyl- $\alpha$-D-mannopyranosyl-( $1 \rightarrow 2)$-3,4,6-tri-Obenzyl- $\alpha$-D-mannopyranoside (16)

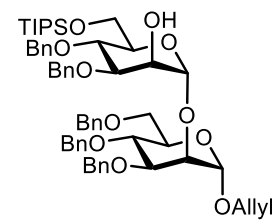

To a stirred solution of dimannoside $11(1.1 \mathrm{~g}, 1.07 \mathrm{mmol})$ in $\mathrm{MeOH}(12 \mathrm{~mL})$ was added freshly prepared $1 \mathrm{M}$ solution of $\mathrm{NaOMe}$. After $1 \mathrm{~h}$, the reaction was neutralized with Amberlite IR $120 \mathrm{H}^{+}$resin, filtered and concentrated to obtain crude product $16(0.96 \mathrm{~g}, 0.97 \mathrm{mmol}, 91 \%)$. $\mathrm{R}_{f}=0.35\left(\right.$ EtOAc/Hexanes 1:3) ${ }^{1} \mathrm{H}$ NMR $\left(400 \mathrm{MHz}, \mathrm{CDCl}_{3}\right) \delta 7.38-7.23(\mathrm{~m}, 28 \mathrm{H}), 7.27-$ $7.15(\mathrm{~m}, 5 \mathrm{H}), 5.85(\mathrm{ddt}, J=16.3,10.9,5.5 \mathrm{~Hz}, 1 \mathrm{H}), 5.25(\mathrm{dd}, J=3.5,1.5 \mathrm{~Hz}, 2 \mathrm{H}), 5.20(\mathrm{~d}, J=$ $1.5 \mathrm{~Hz}, 1 \mathrm{H}), 5.17-5.12(\mathrm{~m}, 2 \mathrm{H}), 4.91-4.80(\mathrm{~m}, 4 \mathrm{H}), 4.76-4.58(\mathrm{~m}, 5 \mathrm{H}), 4.60-4.49(\mathrm{~m}$, $4 \mathrm{H}), 4.14(\mathrm{dd}, J=13.5,4.5 \mathrm{~Hz}, 4 \mathrm{H}), 4.00-3.66(\mathrm{~m}, 15 \mathrm{H}), 1.06(\mathrm{~d}, J=4.3 \mathrm{~Hz}, 21 \mathrm{H}) .{ }^{13} \mathrm{C} \mathrm{NMR}$ $\left(101 \mathrm{MHz}, \mathrm{CDCl}_{3}\right) \delta 138.5,138.5,138.3,138.1,138.0,133.7,128.4,128.4,128.3,128.3,128.2$, $127.9,127.9,127.8,127.8,127.7,127.6,127.6,127.4,127.4,117.1,100.1,98.2,80.2,80.0$, 
75.1, 75.0, 74.7, 74.1, 73.2, 72.5, 72.1, 72.1, 71.9, 69.2, 68.5, 67.8, 62.9, 18.0, 18.0, 11.9. ESIMS $(\mathrm{m} / \mathrm{z}):[\mathrm{M}+\mathrm{K}]^{+}$cald 1027.479 obsd 1027.291.

n-Allyl-2-O-acetyl-3,4,6-tri-O-benzyl- $\alpha$-D-mannopyranosyl- $(1 \rightarrow 2)-3,4-d i-O-b e n z y l-6-O-$ triisopropylsilyl- $\alpha$-D-mannopyranosyl- $(1 \rightarrow 2)$-3,4,6-tri-O-benzyl- $\alpha$-D-mannopyranoside $(17)$

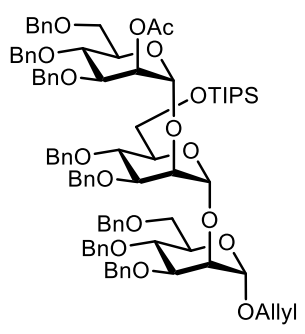

The mannosyl imidate $(0.19 \mathrm{~g}, 0.30 \mathrm{mmol})$ and disaccharide acceptor $16(0.20 \mathrm{~g}, 0.20 \mathrm{mmol})$ were co-evaporated with toluene $(5 \mathrm{~mL} \times 3)$ and dried under high vacuum for $2 \mathrm{~h}$. The mixture was dissolved in anhydrous diethylether $(8 \mathrm{~mL})$ and activated molecular sieves were added. The solution was stirred for $10 \mathrm{~min}$ at room temperature and cooled to $0{ }^{\circ} \mathrm{C}$. The mixture was treated with TBSOTf $(14 \mu \mathrm{L}, 0.06 \mathrm{mmol})$ and stirred at $0{ }^{\circ} \mathrm{C}$ for a period of $1 \mathrm{~h}$. The reaction was diluted with $\mathrm{CH}_{2} \mathrm{Cl}_{2}$, quenched with $\mathrm{Et}_{3} \mathrm{~N}$ and concentrated. The crude product was purified by flash column chromatography to obtain mannosyl trisaccharide $17(0.2 \mathrm{~g}, 0.14 \mathrm{mmol}, 68 \%)$ as colorless oil. $\mathrm{R}_{f}=0.4\left(\right.$ EtOAc/Hexanes 1:4) ${ }^{1} \mathrm{H}$ NMR $\left(400 \mathrm{MHz}, \mathrm{CDCl}_{3}\right) \delta 7.40-7.27(\mathrm{~m}$, $36 \mathrm{H}), 7.30-7.16(\mathrm{~m}, 39 \mathrm{H}), 7.15(\mathrm{dd}, J=16.8,5.4 \mathrm{~Hz}, 6 \mathrm{H}), 5.86$ (ddt, $J=16.3,10.8,5.5 \mathrm{~Hz}$, $1 \mathrm{H}), 5.55(\mathrm{~s}, 1 \mathrm{H}), 5.30(\mathrm{~s}, 1 \mathrm{H}), 5.27-5.11(\mathrm{~m}, 3 \mathrm{H}), 5.03(\mathrm{~s}, 1 \mathrm{H}), 4.88(\mathrm{t}, J=8.2 \mathrm{~Hz}, 3 \mathrm{H}), 4.85$ $-4.71(\mathrm{~m}, 3 \mathrm{H}), 4.67(\mathrm{dd}, J=11.5,6.3 \mathrm{~Hz}, 3 \mathrm{H}), 4.67-4.50(\mathrm{~m}, 9 \mathrm{H}), 4.53-4.46(\mathrm{~m}, 3 \mathrm{H}), 4.50$ $-4.35(\mathrm{~m}, 3 \mathrm{H}), 4.27(\mathrm{dd}, J=12.1,5.4 \mathrm{~Hz}, 1 \mathrm{H}), 4.14(\mathrm{dd}, J=12.9,5.0 \mathrm{~Hz}, 2 \mathrm{H}), 4.06(\mathrm{~s}, 3 \mathrm{H})$, $4.02-3.86(\mathrm{~m}, 13 \mathrm{H}), 3.89-3.83(\mathrm{~m}, 3 \mathrm{H}), 3.77(\mathrm{dt}, J=14.1,10.9 \mathrm{~Hz}, 7 \mathrm{H}), 3.69(\mathrm{~d}, J=9.1 \mathrm{~Hz}$, $3 \mathrm{H}), 3.64-3.54(\mathrm{~m}, 2 \mathrm{H}), 3.46(\mathrm{~d}, J=10.0 \mathrm{~Hz}, 1 \mathrm{H}), 2.13(\mathrm{~s}, 3 \mathrm{H}), 1.09-1.01(\mathrm{~m}, 22 \mathrm{H}) .{ }^{13} \mathrm{C}$ NMR $\left(101 \mathrm{MHz}, \mathrm{CDCl}_{3}\right) \delta 170.1,138.6,138.6,138.5,138.4,138.3,138.2,138.1,138.1,133.7$, $128.3,128.3,128.3,128.2,128.2,128.1,128.0,128.0,127.9,127.7,127.7,127.7,127.6,127.5$, $127.5,127.4,117.1,100.0,99.8,98.1,80.0,78.4,75.1,75.0,74.7,74.2,73.5,73.3,73.3,72.1$, $71.9,71.8,71.6,69.2,68.7,67.7,26.0,21.1,18.1,11.9$. ESI-MS $(\mathrm{m} / \mathrm{z}):[\mathrm{M}+\mathrm{K}]^{+}$cald 1501.683 obsd 1501.592 .

2-O-acetyl-3,4,6-tri-O-benzyl- $\alpha$-D-mannopyranosyl-(1 $\rightarrow 2)-3,4-d i-O-b e n z y l-6-O-$ triisopropylsilyl- $\alpha$-D-mannopyranosyl-(1 $\rightarrow 2)$-3,4,6-tri-O-benzyl- $\alpha$-D-mannopyranosyl trichloroacetimidate (18)

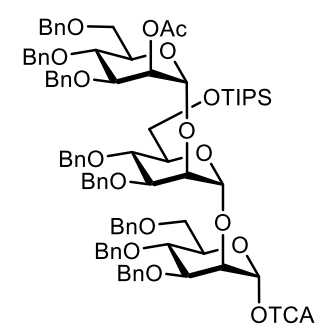

A solution of $\left[\operatorname{IrCOD}\left(\mathrm{PPh}_{2} \mathrm{Me}\right)_{2}\right] \mathrm{PF}_{6}(5.0 \mathrm{mg})$ in THF $(3 \mathrm{~mL})$ was stirred under hydrogen atmosphere until the color turned from red to colorless to pale yellow. The hydrogen atmosphere was exchanged with Argon. This solution was added to a solution of trisaccharide $17(0.18 \mathrm{~g}, 0.13 \mathrm{mmol})$ in THF $(10 \mathrm{~mL})$. After $16 \mathrm{~h}$, the solvent was removed and the residue was dissolved in a mixture of acetone $(2.7 \mathrm{~mL})$ and water $(0.3 \mathrm{~mL})$. Mercury (II) chloride $(0.17$ 
g, $0.63 \mathrm{mmol}$ ) and mercury (II) oxide (5.5 $\mathrm{mg}, 0.03 \mathrm{mmol})$ were added. After $1 \mathrm{~h}$, saturated $\mathrm{NaHCO}_{3}$ (aq) was added and the reaction mixture was extracted three times with $\mathrm{CH}_{2} \mathrm{Cl}_{2}$. The combined organic layers were dried over $\mathrm{Na}_{2} \mathrm{SO}_{4}$ and concentrated. The crude product was purified by flash column chromatography to give trisaccharide hemiacetal intermediate $(0.12$ g, $0.08 \mathrm{mmol}, 64 \%)$ as colorless oil. ${ }^{1} \mathrm{H} \mathrm{NMR}\left(400 \mathrm{MHz}, \mathrm{CDCl}_{3}\right) \delta 7.30-7.21(\mathrm{~m}, 25 \mathrm{H}), 7.23$ $-7.12(\mathrm{~m}, 33 \mathrm{H}), 7.16-7.02(\mathrm{~m}, 13 \mathrm{H}), 5.47(\mathrm{dd}, J=2.9,1.7 \mathrm{~Hz}, 2 \mathrm{H}), 5.23(\mathrm{~d}, J=7.7 \mathrm{~Hz}, 2 \mathrm{H})$, $4.93(\mathrm{~s}, 1 \mathrm{H}), 4.86-4.66(\mathrm{~m}, 7 \mathrm{H}), 4.68-4.32(\mathrm{~m}, 20 \mathrm{H}), 4.29-4.14(\mathrm{~m}, 2 \mathrm{H}), 4.06(\mathrm{dd}, J=5.6$, $2.3 \mathrm{~Hz}, 1 \mathrm{H}), 3.97(\mathrm{~s}, 1 \mathrm{H}), 3.94-3.67(\mathrm{~m}, 18 \mathrm{H}), 3.67-3.59(\mathrm{~m}, 6 \mathrm{H}), 3.56$ (ddd, $J=19.2,9.9$, $3.5 \mathrm{~Hz}, 7 \mathrm{H}), 3.42(\mathrm{dd}, J=10.3,6.3 \mathrm{~Hz}, 2 \mathrm{H}), 2.06(\mathrm{~s}, 3 \mathrm{H}), 1.00(\mathrm{~d}, J=2.1 \mathrm{~Hz}, 22 \mathrm{H}) .{ }^{13} \mathrm{C} \mathrm{NMR}$ $\left(101 \mathrm{MHz}, \mathrm{CDCl}_{3}\right) \delta 170.2,138.6,138.5,138.4,138.2,138.1,128.4,128.4,128.3,128.3,128.2$, $128.1,128.1,128.0,128.0,127.8,127.7,127.6,127.6,127.6,127.5,127.4,100.0,99.8,99.6$, $79.7,79.2,78.4,75.5,75.1,74.7,74.2,73.75,73.4,73.3,72.1,72.0,71.7,69.3,69.1,68.7,68.6$, $62.9,62.8,34.8,31.9,29.7,26.1,22.7,21.2,18.2,14.2,11.9$.

To a stirred solution of hemiacetal $(0.11 \mathrm{~g}, 0.08 \mathrm{mmol})$ in $\mathrm{CH}_{2} \mathrm{Cl}_{2}(10 \mathrm{~mL})$ at $0{ }^{\circ} \mathrm{C}$ were added $\mathrm{CCl}_{3} \mathrm{CN}(0.08 \mathrm{~mL}, 0.77 \mathrm{mmol})$ and $\mathrm{DBU}(4 \mu \mathrm{L}, 0.02 \mathrm{mmol})$. The reaction mixture was stirred for $1 \mathrm{~h}$ at $0{ }^{\circ} \mathrm{C}$. The resulting mixture was concentrated and purified by flash column chromatography to obtain imidate donor $18(0.1 \mathrm{~g}, 0.06 \mathrm{mmol}, 80 \%)$ as clear oil. $\mathrm{R}_{f}=0.4$ $($ EtOAc/hexane $=2: 3)$

1-O-(6-thiobenzyl)hexyl-2-O-Acetyl-3,4,6-tri-O-benzyl- $\alpha$-D-mannopyranosyl-(1 $\rightarrow 2)-3,4-d i$ O-benzyl-6-O-triisopropylsilyl- $\alpha$-D-mannopyranosyl-(1 $\rightarrow 2)$-3,4,6-tri-O-benzyl- $\alpha$ - $D$ -

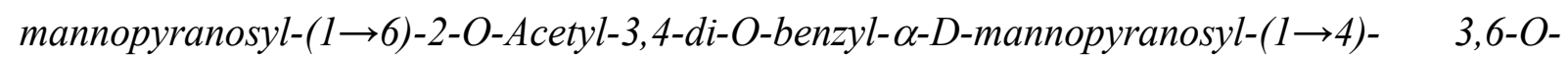
benzyl-2-deoxy-2-N-phthalimido- $\beta$-D-glucopyranoside (19)

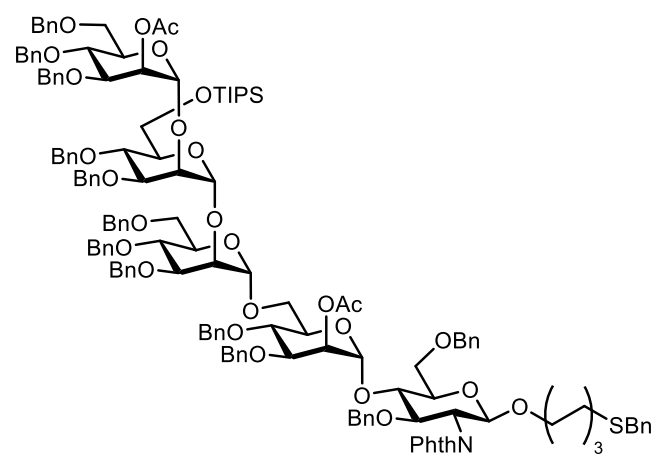

The trisaccharide imidate $18(0.19 \mathrm{~g}, 0.12 \mathrm{mmol})$ and disaccharide acceptor $\mathbf{1 0}(0.11 \mathrm{~g}, 0.10$ mmol) were co-evaporated with toluene $(3 \times 5 \mathrm{~mL})$ and dried under high vacuum for $2 \mathrm{~h}$. The mixture was dissolved in anhydrous diethylether $(10 \mathrm{~mL})$ and activated molecular sieves were added. The solution was stirred for $10 \mathrm{~min}$ at room temperature and cooled to $0{ }^{\circ} \mathrm{C}$. The mixture was treated with TBSOTf $(7 \mu \mathrm{L}, 0.03 \mathrm{mmol})$ and stirred at $0{ }^{\circ} \mathrm{C}$ for a period of $1 \mathrm{~h}$. The reaction was diluted with $\mathrm{CH}_{2} \mathrm{Cl}_{2}$, quenched with $\mathrm{Et}_{3} \mathrm{~N}$ and concentrated. The crude product was purified by flash column chromatography to obtain pentasaccharide $19(0.17 \mathrm{~g}, 0.07 \mathrm{mmol}, 65 \% \alpha$ isomer). $\mathrm{R}_{f}=0.4(\mathrm{EtOAc} /$ hexane $=1: 3){ }^{1} \mathrm{H} \mathrm{NMR}\left(400 \mathrm{MHz}, \mathrm{CDCl}_{3}\right) \delta 7.63(\mathrm{~d}, J=16.2 \mathrm{~Hz}$, $3 \mathrm{H}), 7.34-7.17(\mathrm{~m}, 57 \mathrm{H}), 7.20-7.07(\mathrm{~m}, 12 \mathrm{H}), 7.10-7.04(\mathrm{~m}, 4 \mathrm{H}), 7.03-6.84(\mathrm{~m}, 6 \mathrm{H}), 6.77$ $(\mathrm{t}, J=7.4 \mathrm{~Hz}, 1 \mathrm{H}), 5.52(\mathrm{~d}, J=19.8 \mathrm{~Hz}, 1 \mathrm{H}), 5.30(\mathrm{~s}, 2 \mathrm{H}), 5.06-4.99(\mathrm{~m}, 2 \mathrm{H}), 4.92-4.20(\mathrm{~m}$, $32 \mathrm{H}), 4.15(\mathrm{dd}, J=17.7,7.1 \mathrm{~Hz}, 2 \mathrm{H}), 4.07-3.92(\mathrm{~m}, 6 \mathrm{H}), 3.96-3.80(\mathrm{~m}, 11 \mathrm{H}), 3.75$ (ddd, $J$ $=19.1,15.0,10.8 \mathrm{~Hz}, 11 \mathrm{H}), 3.60(\mathrm{~s}, 2 \mathrm{H}), 3.50-3.22(\mathrm{~m}, 6 \mathrm{H}), 2.13(\mathrm{~d}, J=14.8 \mathrm{~Hz}, 5 \mathrm{H}), 1.93$ $(\mathrm{d}, J=3.4 \mathrm{~Hz}, 3 \mathrm{H}), 1.44-1.17(\mathrm{~m}, 5 \mathrm{H}), 1.21-1.00(\mathrm{~m}, 27 \mathrm{H}), 1.01-0.80(\mathrm{~m}, 5 \mathrm{H}) .{ }^{13} \mathrm{C}$ NMR 
$\left(101 \mathrm{MHz}, \mathrm{CDCl}_{3}\right) \delta 170.1,169.8,138.8,138.7,138.5,138.5,138.4,138.0,137.7,128.7,128.4$, $128.4,128.3,128.3,128.2,128.2,128.2,128.1,128.1,128.1,128.0,128.0,127.9,127.9,127.7$, $127.7,127.6,127.5,127.4,127.4,127.3,127.1,127.0,126.8,100.0,99.9,99.3,99.0,98.0,80.9$, 79.4, 78.6, 78.5, 77.6, 75.0, 74.8, 74.6, 74.5, 74.4, 74.0, 73.3, 73.1, 72.7, 72.1, 72.0, 71.73, 71.4, 71.3, 69.3, 68.9, 68.7, 68.5, 55.7, 36.2, 31.0, 29.6, 29.0, 28.9, 28.3, 26.0, 25.3, 21.1, 20.9, 18.1, 11.9. HRMS $(\mathrm{m} / \mathrm{z}):[\mathrm{M}+\mathrm{Na}]^{+}$cald 2486.3377 obsd 2486.3362

1-O-(6-thiobenzyl)hexyl-2-O-Acetyl-3,4,6-tri-O-benzyl- $\alpha$-D-mannopyranosyl- $(1 \rightarrow 2)-3,4-d i$ O-benzyl- $\alpha$-D-mannopyranosyl- $(1 \rightarrow 2)-3,4,6$-tri-O-benzyl- $\alpha$-D-mannopyranosyl- $(1 \rightarrow 6)-2-O$ Acetyl-3,4-di-O-benzyl- $\alpha$-D-mannopyranosyl-(1 $\rightarrow 4)$-3, 6-O-benzyl-2-deoxy-2-N-phthalimido$\beta$-D-glucopyranoside (20)

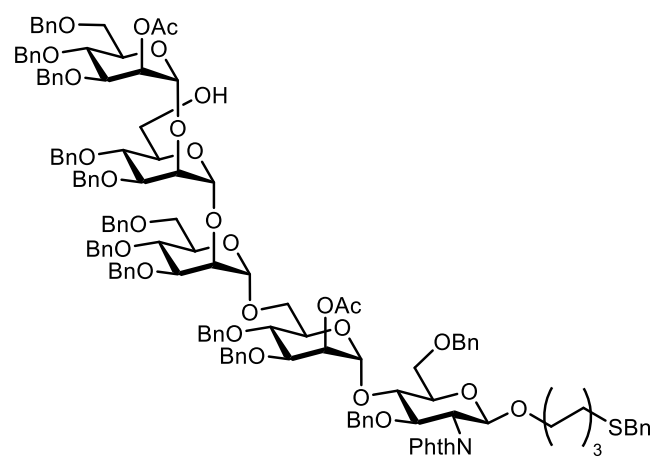

To a stirred solution of pentasaccharide $19(0.07 \mathrm{~g}, 0.03 \mathrm{mmol})$ in $\mathrm{CH}_{3} \mathrm{CN}(7 \mathrm{~mL})$ were added water $(50 \mu \mathrm{L})$ and $\mathrm{Sc}(\mathrm{OTf})_{3}(0.04 \mathrm{~g}, 0.08 \mathrm{mmol})$. The reaction mixture was warmed to $50{ }^{\circ} \mathrm{C}$ and stirred for $6 \mathrm{~h}$. The reaction was quenched with $\mathrm{Et}_{3} \mathrm{~N}$ and concentrated. The crude product was purified by flash column chromatography to obtain $\mathbf{2 0}(50 \mathrm{mg}, 0.02 \mathrm{mmol}, 72 \%)$. $\mathrm{R}_{f}=0.25$ $($ EtOAc/hexane $=2: 3){ }^{1} \mathrm{H}$ NMR $\left(400 \mathrm{MHz}, \mathrm{CDCl}_{3}\right) \delta 7.63(\mathrm{~d}, J=15.8 \mathrm{~Hz}, 4 \mathrm{H}), 7.35-7.13(\mathrm{~m}$, $86 \mathrm{H}), 7.16-7.08(\mathrm{~m}, 11 \mathrm{H}), 7.02(\mathrm{dd}, J=16.1,7.6 \mathrm{~Hz}, 4 \mathrm{H}), 6.88(\mathrm{t}, J=7.5 \mathrm{~Hz}, 3 \mathrm{H}), 6.77(\mathrm{t}, J$ $=7.2 \mathrm{~Hz}, 1 \mathrm{H}), 5.50(\mathrm{~d}, J=18.3 \mathrm{~Hz}, 3 \mathrm{H}), 5.29(\mathrm{~s}, 1 \mathrm{H}), 5.15(\mathrm{~s}, 1 \mathrm{H}), 5.02(\mathrm{~d}, J=9.0 \mathrm{~Hz}, 3 \mathrm{H})$, $4.92-4.75(\mathrm{~m}, 8 \mathrm{H}), 4.76-4.60(\mathrm{~m}, 4 \mathrm{H}), 4.62-4.49(\mathrm{~m}, 11 \mathrm{H}), 4.51-4.39(\mathrm{~m}, 7 \mathrm{H}), 4.42-$ $4.24(\mathrm{~m}, 7 \mathrm{H}), 4.22-4.13(\mathrm{~m}, 1 \mathrm{H}), 4.07(\mathrm{~s}, 2 \mathrm{H}), 3.99-3.57(\mathrm{~m}, 33 \mathrm{H}), 3.58-3.39(\mathrm{~m}, 4 \mathrm{H}), 3.35$ $-3.18(\mathrm{~m}, 1 \mathrm{H}), 2.16(\mathrm{~d}, J=7.1 \mathrm{~Hz}, 1 \mathrm{H}), 2.11(\mathrm{~s}, 4 \mathrm{H}), 1.93(\mathrm{~s}, 3 \mathrm{H}), 1.42-1.22(\mathrm{~m}, 2 \mathrm{H}), 1.21-$ $1.08(\mathrm{~m}, 1 \mathrm{H}), 1.04-0.79(\mathrm{~m}, 1 \mathrm{H}) .{ }^{13} \mathrm{C}$ NMR $\left(101 \mathrm{MHz}, \mathrm{CDCl}_{3}\right) \delta 170.0,169.8,138.4,138.4$, $138.3,138.1,137.9,137.9,137.8,137.8,137.6,128.7,128.4,128.4,128.3,128.3,128.2,128.2$, $128.2,128.1,128.1,128.0,127.9,127.9,127.8,127.7,127.7,127.6,127.6,127.5,127.4,127.4$, $127.3,127.3,127.1,127.0,126.8,100.1,99.3,99.1,98.9,98.0,81.0,78.9,78.3,77.8,75.1$, $75.0,74.8,74.7,74.5,74.4,74.2,74.0,73.6,73.4,73.3,73.2,72.6,72.0,71.9,71.7,71.6,69.3$, $69.2,68.8,68.6,68.5,68.4,65.9,62.0,55.7,36.1,32.3,31.0,29.0,28.9,28.3,26.3,25.3,21.1$, 20.9. ESI-MS $(\mathrm{m} / \mathrm{z}):[\mathrm{M}+\mathrm{Na}]^{+}$cald 2351.983 obsd 2351.651

1-O-(6-thio)hexyl- $\alpha$-D-mannopyranosyl- $(1 \rightarrow 2)-\alpha$-D-mannopyranosyl- $(1 \rightarrow 2)-\alpha-D$ mannopyranosyl- $(1 \rightarrow 6)$ - $\alpha$-D-mannopyranosyl-( $1 \rightarrow 4)$-2-deoxy-2-amino- $\beta$-D-glucopyranoside (3) 


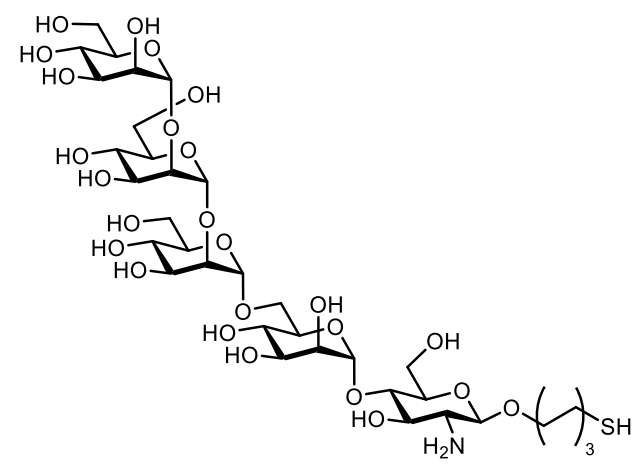

Pentasaccharide $20(0.02 \mathrm{~g}, 8.6 \mu \mathrm{mol})$ was dissolved in a mixture of ethylenediamine $(2 \mathrm{~mL})$ and butanol $(2 \mathrm{~mL})$ and stirred at $90{ }^{\circ} \mathrm{C}$ for $3 \mathrm{~h}$. After $3 \mathrm{~h}$, the reaction mixture was concentrated to obtain partially deprotected crude intermediate. The crude intermediate was dissolved in anhydrous THF and $\mathrm{MeOH}$. This solution was added dropwise to blue solution of $20 \mathrm{~mL}$ liquefied ammonia with sodium $-78{ }^{\circ} \mathrm{C}$. The reaction was stirred at $-78{ }^{\circ} \mathrm{C}$. After $1 \mathrm{~h}$, the reaction was quenched with $\mathrm{MeOH}$ and stirred for additional $1 \mathrm{~h}$ at rt. Sodium methoxide generated in the reaction was quenched by dropwise addition of glacial acetic acid. The reaction mixture was concentrated and the crude product was purified by size exclusion column chromatography using a Sephadex ${ }^{\circledR}$ super fine G-15 (GE Healthcare) column and 5\% ethanol in water as eluent to obtain $3(6 \mathrm{mg}, 6.36 \mu \mathrm{mol}, 74 \%)$ as white solid. ${ }^{1} \mathrm{H}$ NMR $\left(600 \mathrm{MHz}, \mathrm{D}_{2} \mathrm{O}\right)$ $\delta 5.19-5.15(\mathrm{~m}, 1 \mathrm{H}), 5.10-5.06(\mathrm{~m}, 1 \mathrm{H}), 5.00(\mathrm{~s}, 1 \mathrm{H}), 4.92(\mathrm{~d}, J=1.5 \mathrm{~Hz}, 1 \mathrm{H}), 4.61-4.52$ $(\mathrm{m}, 1 \mathrm{H}), 4.01-3.96(\mathrm{~m}, 1 \mathrm{H}), 3.94(\mathrm{~s}, 2 \mathrm{H}), 3.90-3.80(\mathrm{~m}, 9 \mathrm{H}), 3.80-3.41(\mathrm{~m}, 28 \mathrm{H}), 2.96-$ $2.88(\mathrm{~m}, 1 \mathrm{H}), 2.84-2.72(\mathrm{~m}, 1 \mathrm{H}), 2.27-2.21(\mathrm{~m}, 1 \mathrm{H}), 1.55-1.50(\mathrm{~m}, 2 \mathrm{H}), 1.46(\mathrm{p}, J=7.4$ $\mathrm{Hz}, 1 \mathrm{H}), 1.30$ (ddd, $J=28.5,14.3,7.2 \mathrm{~Hz}, 4 \mathrm{H}) .{ }^{13} \mathrm{C} \mathrm{NMR}\left(151 \mathrm{MHz}, \mathrm{d}_{2} \mathrm{o}\right) \delta 104.8,104.5,103.2$, $101.2,100.9,81.4,81.1,79.7,77.5,75.8,75.3,74.9,73.2,72.9,72.7,72.5,69.6,69.5,69.4$, $68.9,63.7,63.5,63.2,63.2,58.4,53.4,30.9,30.4,29.8,27.3,26.4,25.4,24.1$. HRMS $(\mathrm{m} / z)$ : $[\mathrm{M}+\mathrm{H}]^{+}$cald 944.3639 obsd 944.3621

\section{Synthesis of fragment 4}

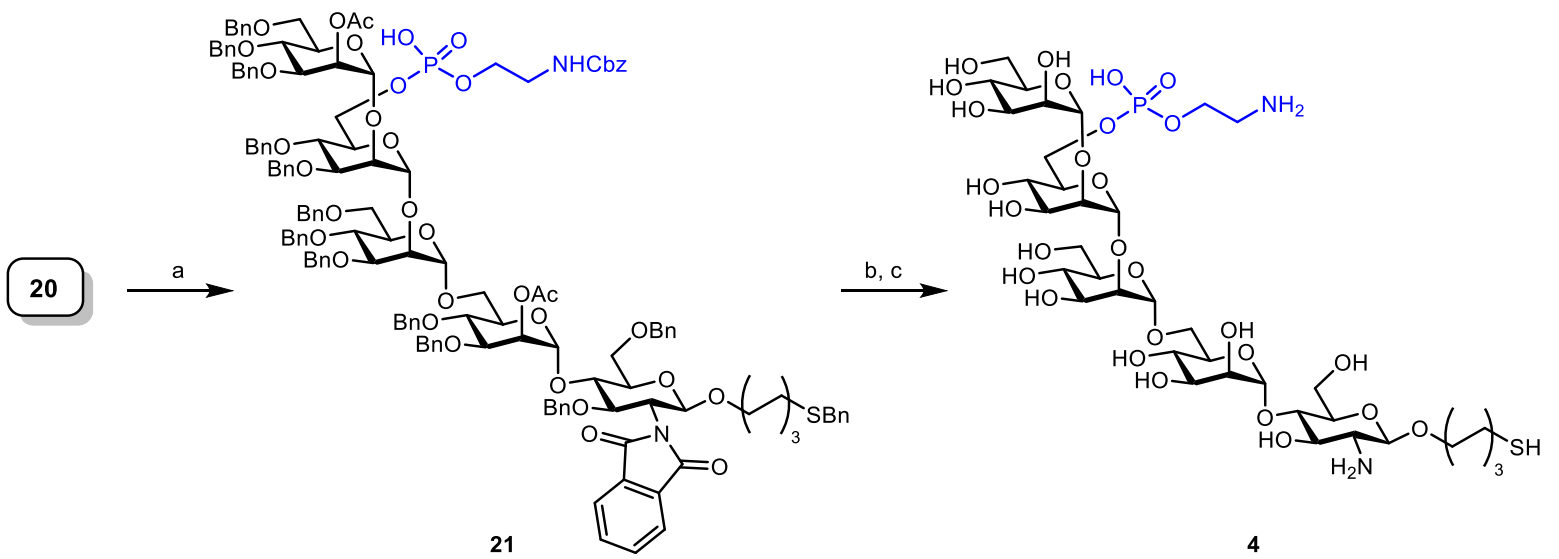

Scheme S5: Synthesis of fragment GPI 4. a) i. 2-3, PivCl, py, rt, 16 h; ii. $\mathrm{I}_{2}, \mathrm{H}_{2} \mathrm{O}$, pyr, rt, $1 \mathrm{~h}, 86 \%$ b) $\left(\mathrm{CH}_{2} \mathrm{NH}_{2}\right)_{2}, \mathrm{H}_{2} \mathrm{O}, n-\mathrm{BuOH}, 90^{\circ} \mathrm{C}, 4 \mathrm{~h}$; c) i. Na, liq. $\mathrm{NH}_{3}$, THF, $-78^{\circ} \mathrm{C}, 1 \mathrm{~h}$; ii. $\mathrm{MeOH}, \mathrm{rt}, 1 \mathrm{~h}, 60 \%$ (over two steps).

1-O-(6-thiobenzyl)hexyl-2-O-Acetyl-3,4,6-tri-O-benzyl- $\alpha$-D-mannopyranosyl-(1 $\rightarrow 2)-3,4-d i$ O-benzyl-6-O-(2-N-benzyloxycarbonyl) aminoethyl-phosphonato- $\alpha$-D-mannopyranosyl- 
$(1 \rightarrow 2)-3,4,6$-tri-O-benzyl- $\alpha$-D-mannopyranosyl-(1 $\rightarrow 6)-2-O$-Acetyl-3,4-di-O-benzyl- $\alpha-D$ mannopyranosyl-(1 $\rightarrow 4)$-3,6-O-benzyl-2-deoxy-2-N-phthalimido- $\beta$-D-glucopyranoside (21)

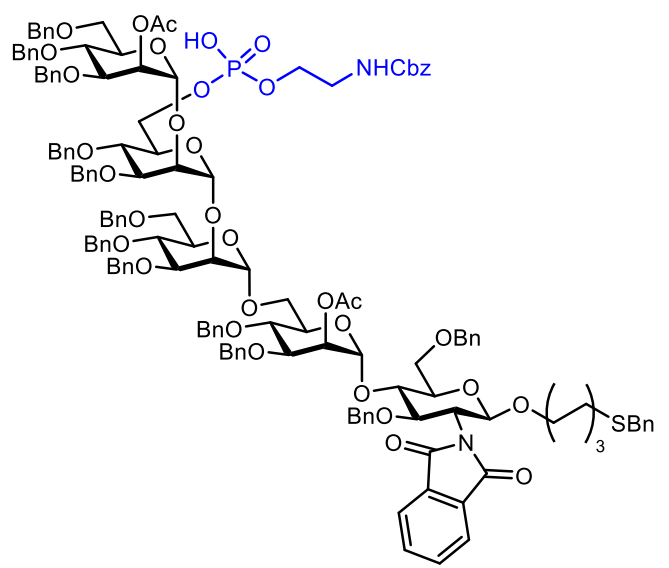

Pentasaccharide 20 (0.03 g, $0.01 \mathrm{mmol})$ and $H$-phosphonatethanolamine ( $0.02 \mathrm{~g}, 0.04 \mathrm{mmol})$ were co-evaporated with pyridine for three times and dried under high vacuum for $2 \mathrm{~h}$. The mixture was dissolved in anhydrous pyridine $(4 \mathrm{~mL})$ and a solution of pivolyl chloride $(5 \mu \mathrm{L}$, $0.04 \mathrm{mmol})$ in pyridine $(1 \mathrm{~mL})$ was added. The solution was stirred for $6 \mathrm{~h}$ at room temperature. After $6 \mathrm{~h}$, iodine $(0.01 \mathrm{~g}, 0.04 \mathrm{mmol})$ and water $(0.05 \mathrm{~mL})$ were added and reaction was stirred for $2 \mathrm{~h}$. The reaction mixture was quenched with $\mathrm{Na}_{2} \mathrm{~S}_{2} \mathrm{O}_{3}$ and extracted with $\mathrm{CH}_{2} \mathrm{Cl}_{2}$. The organic layer were concentrated and purified by $\mathrm{Et}_{3} \mathrm{~N}$ deactivated silica gel flash column chromatography to obtain phosphorylated pentasaccharide $21(0.03 \mathrm{~g}, 0.01 \mathrm{mmol}, 86 \%)$. $\mathrm{R}_{f}=$ $0.5\left(\mathrm{MeOH} / \mathrm{CH}_{2} \mathrm{Cl}_{2}=1: 10\right){ }^{1} \mathrm{H}$ NMR $\left(400 \mathrm{MHz}, \mathrm{CDCl}_{3}\right) \delta 7.63(\mathrm{~d}, J=16.2 \mathrm{~Hz}, 6 \mathrm{H}), 7.33(\mathrm{~d}$, $J=7.7 \mathrm{~Hz}, 5 \mathrm{H}), 7.32-7.24(\mathrm{~m}, 15 \mathrm{H}), 7.25-7.14(\mathrm{~m}, 57 \mathrm{H}), 7.14(\mathrm{~d}, J=3.2 \mathrm{~Hz}, 5 \mathrm{H}), 7.11(\mathrm{~d}$, $J=7.1 \mathrm{~Hz}, 6 \mathrm{H}), 7.08-7.02(\mathrm{~m}, 4 \mathrm{H}), 6.97(\mathrm{~d}, J=7.3 \mathrm{~Hz}, 4 \mathrm{H}), 6.87$ (t, $J=7.6 \mathrm{~Hz}, 2 \mathrm{H}), 6.78(\mathrm{~d}$, $J=5.7 \mathrm{~Hz}, 2 \mathrm{H}), 5.53(\mathrm{~d}, J=25.7 \mathrm{~Hz}, 3 \mathrm{H}), 5.29(\mathrm{~s}, 1 \mathrm{H}), 5.20(\mathrm{~s}, 1 \mathrm{H}), 5.00(\mathrm{td}, J=23.2,22.0$, $14.0 \mathrm{~Hz}, 4 \mathrm{H}), 4.79(\mathrm{dt}, J=21.0,11.9 \mathrm{~Hz}, 11 \mathrm{H}), 4.68-4.22(\mathrm{~m}, 28 \mathrm{H}), 4.21-4.06(\mathrm{~m}, 4 \mathrm{H}), 4.02$ $-3.68(\mathrm{~m}, 27 \mathrm{H}), 3.60(\mathrm{~d}, J=7.8 \mathrm{~Hz}, 4 \mathrm{H}), 3.51-3.36(\mathrm{~m}, 5 \mathrm{H}), 3.29(\mathrm{~s}, 3 \mathrm{H}), 2.14(\mathrm{t}, J=7.3 \mathrm{~Hz}$, $2 \mathrm{H}), 1.95(\mathrm{~s}, 3 \mathrm{H}), 1.40-1.21(\mathrm{~m}, 5 \mathrm{H}), 1.07-0.83(\mathrm{~m}, 3 \mathrm{H}) .{ }^{13} \mathrm{C}$ NMR $\left(101 \mathrm{MHz}, \mathrm{CDCl}_{3}\right) \delta$ 170.0, 169.9, 156.4, 138.7, 138.4, 138.1, 138.0, 137.7, 137.6, 137.0, 133.8, 128.7, 128.4, 128.4, $128.3,128.2$, 128.1, 127.9, 127.8, 127.8, 127.7, 127.5, 127.47, 127.4, 127.3, 127.2, 126.8, 100.2, 99.4, 99.3, 98.8, 98.0, 80.9, 78.4, 75.1, 74.9, 74.8, 74.6, 74.5, 74.4, 74.0, 73.5, 73.3, 73.1, 72.1, 72.0, 71.9, 71.7, 71.5, 71.3, 69.3, 68.5, 66.1, 55.8, 45.5, 36.1, 31.0, 29.6, 29.0, 28.8, 28.3, 25.3, 21.1, 20.9, 8.8. ${ }^{31} \mathrm{P}$ NMR $\left(162 \mathrm{MHz}, \mathrm{CDCl}_{3}\right) \delta 1.58$. ESI-MS $(\mathrm{m} / \mathrm{z}):[\mathrm{M}+\mathrm{Na}]^{+}$cald 2609.03 obsd 2609.6

1-O-(6-thio)hexyl- $\alpha$-D-mannopyranosyl-( $1 \rightarrow 2)$-6-O-aminoethyl-phosphonato- $\alpha$ - $D$ mannopyranosyl- $(1 \rightarrow 2)$ - $\alpha$-D-mannopyranosyl- $(1 \rightarrow 6)$ - $\alpha$-D-mannopyranosyl- $(1 \rightarrow 4)-2-d e o x y$ 2-amino- $\beta$-D-glucopyranoside (4)

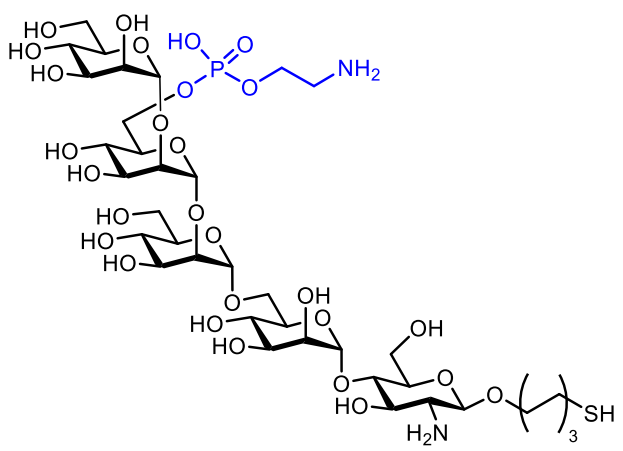


Pentasaccharide 21 (0.04 g, $0.02 \mathrm{mmol})$ was dissolved in a mixture of ethylenediamine $(2 \mathrm{~mL})$ and butanol $(2 \mathrm{~mL})$ and stirred at $90{ }^{\circ} \mathrm{C}$ for $3 \mathrm{~h}$. After $3 \mathrm{~h}$, the reaction mixture was concentrated to obtain partially deprotected crude intermediate. The crude intermediate was dissolved in anhydrous THF and $\mathrm{MeOH}$. This solution was added dropwise to blue solution of $20 \mathrm{~mL}$ liquefied ammonia with sodium $-78{ }^{\circ} \mathrm{C}$. The reaction was stirred at $-78{ }^{\circ} \mathrm{C}$. After $1 \mathrm{~h}$, the reaction was quenched with $\mathrm{MeOH}$ and stirred for additional $1 \mathrm{~h}$ at $\mathrm{rt}$. Sodium methoxide generated in the reaction was quenched by dropwise addition of glacial acetic acid. The reaction mixture was concentrated and the crude product was purified by size exclusion column chromatography using a Sephadex ${ }^{\circledR}$ super fine G-15 (GE Healthcare) column and 5\% ethanol in water as eluent to obtain $4(11 \mathrm{mg}, 10.2 \mu \mathrm{mol}, 60 \%)$ as white solid. ${ }^{1} \mathrm{H}$ NMR $(600 \mathrm{MHz}$, $\left.\mathrm{D}_{2} \mathrm{O}\right) \delta 5.18-5.15(\mathrm{~m}, 1 \mathrm{H}), 5.08(\mathrm{~s}, 1 \mathrm{H}), 4.97(\mathrm{~s}, 1 \mathrm{H}), 4.94-4.90(\mathrm{~m}, 1 \mathrm{H}), 4.32(\mathrm{ddd}, J=19.6$, 7.9, $3.7 \mathrm{~Hz}, 1 \mathrm{H}), 4.03-3.95(\mathrm{~m}, 5 \mathrm{H}), 3.94(\mathrm{dt}, J=5.6,2.7 \mathrm{~Hz}, 2 \mathrm{H}), 3.88-3.77(\mathrm{~m}, 6 \mathrm{H}), 3.76$ $(\mathrm{d}, J=10.7 \mathrm{~Hz}, 2 \mathrm{H}), 3.74-3.51(\mathrm{~m}, 14 \mathrm{H}), 3.48(\mathrm{q}, J=8.8,7.7 \mathrm{~Hz}, 2 \mathrm{H}), 3.47-3.41(\mathrm{~m}, 2 \mathrm{H})$, $3.21-3.14(\mathrm{~m}, 2 \mathrm{H}), 3.01(\mathrm{dt}, J=17.5,6.4 \mathrm{~Hz}, 1 \mathrm{H}), 2.87(\mathrm{dd}, J=10.2,5.3 \mathrm{~Hz}, 0 \mathrm{H}), 2.65(\mathrm{t}, J$ $=7.2 \mathrm{~Hz}, 1 \mathrm{H}), 2.42(\mathrm{t}, J=7.1 \mathrm{~Hz}, 0 \mathrm{H}), 1.62-1.45(\mathrm{~m}, 3 \mathrm{H}), 1.32-1.23(\mathrm{~m}, 2 \mathrm{H}) .{ }^{13} \mathrm{C} \mathrm{NMR}$ $\left(151 \mathrm{MHz}, \mathrm{D}_{2} \mathrm{O}\right) \delta 184.0,104.8,104.4,103.3,100.9,81.5,81.0,79.8,77.4,75.8,75.3,74.9$, $74.7,74.7,73.2,72.9,72.7,72.6,72.4,69.4,69.1,68.9,67.2,64.4,63.6,63.4,58.9,42.7,42.6$, 40.7, 31.1, 30.7, 29.8, 27.2, 27.1, 25.8. ${ }^{31} \mathrm{P}$ NMR (243 MHz, $\left.\mathrm{D}_{2} \mathrm{O}\right) \delta-2.75$. HRMS ESI-MS $(\mathrm{m} / \mathrm{z}):[\mathrm{M}+\mathrm{H}]^{+}$cald 1066.3652 obsd 1066.3646

\section{Synthesis of fragment 5}

2-O-Acetyl-3, 4,6-O-tri-benzyl- $\alpha$-D-mannopyranosyl-(1 $\rightarrow 2)-3,4-O-d i-b e n z y l-6-O-(t r i-$ isopropylsilyl)- $\alpha$-D-mannopyranosyl- $(1 \rightarrow 6)$-2,3,4-tri-O-benzyl- $\alpha$-D-mannopyranosyl- $(1 \rightarrow 4)$ -

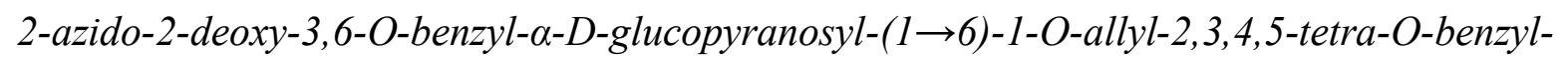
D-myo-inositol (25)

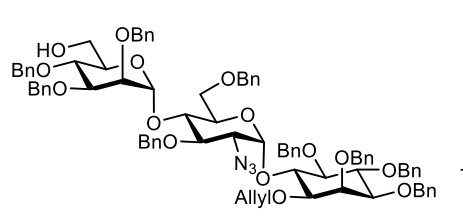

23

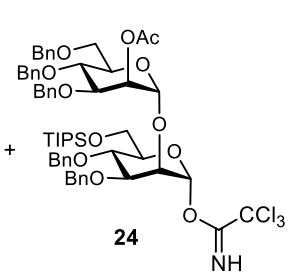

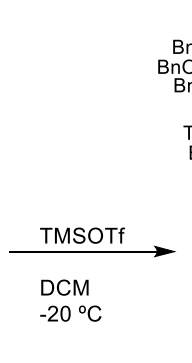

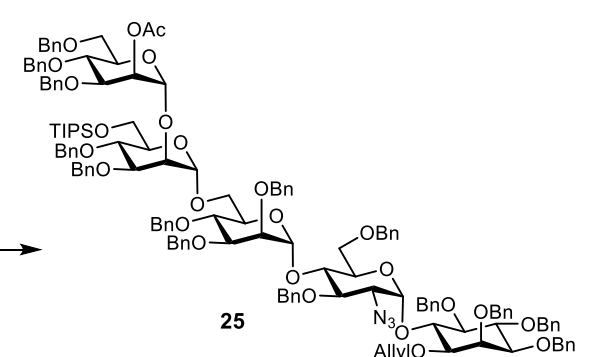

The dimannose imidate $\mathbf{2 4}$ (74 $\mathrm{mg}, 0.065 \mathrm{mmol}$ ) and the trisaccharide acceptor $\mathbf{2 3}^{5}$ (69 $\mathrm{mg}$, $0.050 \mathrm{mmol}$ ) were co-evaporated with toluene 3 times and dry in high-vacuum overnight. The dry remaining was dissolved in DCM, activated 4 A molecular sieves were added (200 $\mathrm{mg}$ ) and the mixture was stirred for 30 minutes at room temperature and then $15 \mathrm{~min}$ at $-40{ }^{\circ} \mathrm{C}$. TMSOTf was added slowly and the reaction mixture was allowed to gradually warm up to $-20^{\circ} \mathrm{C}$. After $1 \mathrm{~h}$ at $-20^{\circ} \mathrm{C}$ TLC indicated completion and the reaction was quenched with addition of $\mathrm{Et}_{3} \mathrm{~N}$. The sieves were removed on celite and the solution was concentrated. The crude mixture was purified on a silica gel column with a gradient from 5\% to $30 \%$ EtOAc in hexane to obtain 92 mg $(0.039 \mathrm{mmol}, 78 \%)$ of the pentasaccharide 25. $\mathrm{R} f=0.52\left(\mathrm{CH}_{2} \mathrm{Cl}_{2} / \mathrm{MeOH}, 10: 1\right),{ }^{1} \mathrm{H}$ NMR $\left(400 \mathrm{MHz}, \mathrm{CDCl}_{3}-d\right) \delta 7.48-7.41(\mathrm{~m}, 2 \mathrm{H}), 7.40-7.06(\mathrm{~m}, 80 \mathrm{H}), 6.02-5.89(\mathrm{~m}, 1 \mathrm{H}), 5.79$ $(\mathrm{d}, J=3.7 \mathrm{~Hz}, 1 \mathrm{H}), 5.55(\mathrm{t}, J=2.4 \mathrm{~Hz}, 1 \mathrm{H}), 5.34(\mathrm{dd}, J=10.8,2.1 \mathrm{~Hz}, 1 \mathrm{H}), 5.27(\mathrm{dd}, J=4.1$, $1.9 \mathrm{~Hz}, 2 \mathrm{H}), 5.21(\mathrm{dd}, J=10.5,1.6 \mathrm{~Hz}, 1 \mathrm{H}), 5.11-4.91(\mathrm{~m}, 6 \mathrm{H}), 4.91-4.81(\mathrm{~m}, 6 \mathrm{H}), 4.81-$ 
$4.59(\mathrm{~m}, 10 \mathrm{H}), 4.59-4.41(\mathrm{~m}, 9 \mathrm{H}), 4.41-4.21(\mathrm{~m}, 6 \mathrm{H}), 4.21-3.73(\mathrm{~m}, 22 \mathrm{H}), 3.73-3.35(\mathrm{~m}$, $12 \mathrm{H}), 3.34-3.20(\mathrm{~m}, 3 \mathrm{H}), 2.12(\mathrm{~s}, 3 \mathrm{H}), 1.04-0.97(\mathrm{~m}, 21 \mathrm{H}),{ }^{13} \mathrm{C} \mathrm{NMR}\left(101 \mathrm{MHz}, \mathrm{CDCl}_{3}\right) \delta$ : 134.2, 128.1, 127.9, 127.8, 127.4, 125.3, 125.1, 97.5, 117.2, 117.1, 117.1, 100.5, 99.8, 99.3, $97.7,81.9,81.2,79.8,79.7,78.6,77.8,77.2,76.2,75.8,75.7,75.5,75.4,75.2,74.9,74.9,74.7$, $74.3,74.2,74.1,74.0,73.8,73.5,73.3,73.0,72.7,72.7,72.2,72.1,72.0,71.9,71.8,70.8,69.7$, 69.6, 68.8, 68.7, 68.6, 68.6, 68.5, 66.4, 63.2, 62.3, 21.2, 19.8, 18.1, 12,0, HRMS ESI-MS ${ }^{+} \mathrm{M}_{\text {Calcd }}$ $\left(\mathrm{C}_{142} \mathrm{H}_{161} \mathrm{~N}_{3} \mathrm{O}_{26} \mathrm{Si}\right): 2352.1138 ; \mathrm{M}_{\text {found }}: 2375.210(\mathrm{M}+\mathrm{Na})^{+}$.

2-O-Acetyl-3,4,6-O-tri-benzyl- $\alpha$-D-mannopyranosyl-( $1 \rightarrow 2)$-3,4-O-di-benzyl-6-O-(triisopropylsilyl)- $\alpha$-D-mannopyranosyl- $(1 \rightarrow 6)-2,3,4$-tri-O-benzyl- $\alpha$-D-mannopyranosyl- $(1 \rightarrow 4)$ 2-azido-2-deoxy-3,6-O-benzyl- $\alpha$-D-glucopyranosyl-(1 $\rightarrow 6)$-1-hydroxy-2,3,4,5-tetra-O-benzylD-myo-inositol (26)

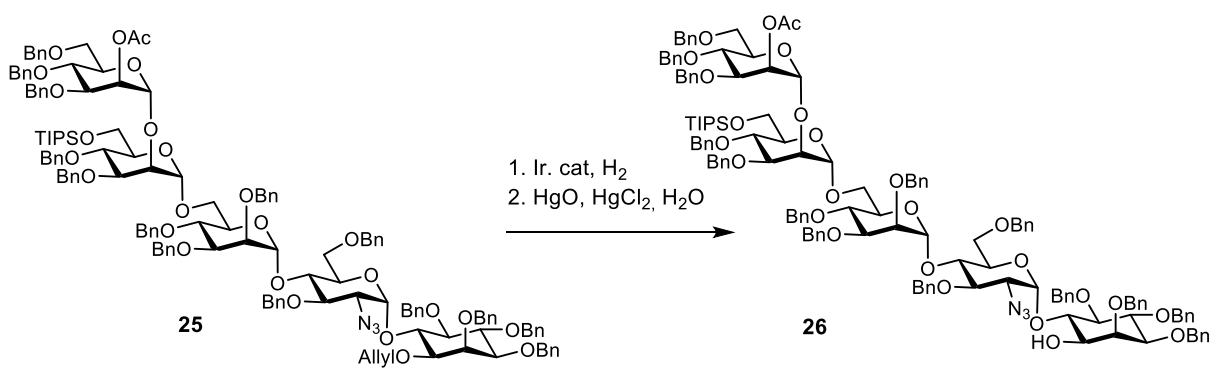

A solution of iridium catalyst $(6.45 \mathrm{mg}, 7.5 \mu \mathrm{mol})$ in THF $(6 \mathrm{~mL})$ was stirred for $15 \mathrm{~min}$ under hydrogen at $20{ }^{\circ} \mathrm{C}$ until the color turned from red to pale yellow. The $\mathrm{H}_{2}$ atmosphere was exchanged with argon and this solution was added pseudo-pentasaccharide 25 (82 $\mathrm{mg}, 0.034$ $\mathrm{mmol}$ ). After $16 \mathrm{~h}$, the solvent was removed and the residue was dissolved in $6 \mathrm{~mL}$ of acetone containing water $(0.3 \mathrm{ml})$. Mercury (II) chloride $(83 \mathrm{mg}, 0.306 \mathrm{mmol})$ and mercury (II) oxide $(2.76 \mathrm{mg}, 0.013 \mathrm{mmol})$ were added. After $2 \mathrm{~h}$, and not additional progress of the reaction, $\mathrm{NaHCO}_{3}$ was added and the reaction mixture was extracted with DCM for three times. The combined organic layers were dried over $\mathrm{Na}_{2} \mathrm{SO}_{4}$, filtered and concentrated in vacuo. The crude product was purified by silica gel column chromatography using n-hexane/EtOAc from 9:1 to $3: 1$ to give $58 \mathrm{mg}(0.025 \mathrm{mmol})$ of the pseudo-pentasaccharide alcohol in $75 \%$ yield as a white powder. $\mathrm{R} f=0.20$ (n-hexan/AcOEt, 2:1), ${ }^{1} \mathrm{H}$ NMR $\left(400 \mathrm{MHz}, \mathrm{CDCl}_{3}\right) \delta 7.43-7.11(\mathrm{~m}, 70 \mathrm{H})$, $5.54(\mathrm{~d}, J=3.3 \mathrm{~Hz}, 2 \mathrm{H}), 5.27(\mathrm{~d}, J=2.1 \mathrm{~Hz}, 1 \mathrm{H}), 5.05(\mathrm{~d}, J=2.2 \mathrm{~Hz}, 1 \mathrm{H}), 5.01(\mathrm{~d}, J=11.6$ $\mathrm{Hz}, 1 \mathrm{H}), 4.97-4.90(\mathrm{~m}, 3 \mathrm{H}), 4.89-4.82(\mathrm{~m}, 4 \mathrm{H}), 4.80-4.69(\mathrm{~m}, 6 \mathrm{H}), 4.66-4.59(\mathrm{~m}, 3 \mathrm{H})$, $4.57-4.41(\mathrm{~m}, 7 \mathrm{H}), 4.40-4.17(\mathrm{~m}, 5 \mathrm{H}), 4.16-3.74(\mathrm{~m}, 15 \mathrm{H}), 3.75-3.55(\mathrm{~m}, 6 \mathrm{H}), 3.55-$ $3.28(\mathrm{~m}, 8 \mathrm{H}), 3.02(\mathrm{~d}, J=7.4 \mathrm{~Hz}, 1 \mathrm{H}), 2.12(\mathrm{~s}, 3 \mathrm{H}), 1.00(\mathrm{~d}, J=6.4 \mathrm{~Hz}, 19 \mathrm{H}), 0.92-0.78(\mathrm{~m}$, $2 \mathrm{H}),{ }^{13} \mathrm{C}$ NMR $\left(101 \mathrm{MHz}, \mathrm{CDCl}_{3}\right) \delta 170.2(\mathrm{C}=\mathrm{O}), 139.0,138.9,138.7,138.5,138.4,138.4$, $138.3,138.3,138.2,138.2,138.1,137.7,128.5,128.5,128.3,128.3,128.3,128.2,128.2,128.1$, $128.0,128.0,127.8,127.8,127.8,127.7,127.7,127.6,127.6,127.5,127.5,127.4,127.3,127.3$, $127.2,127.1,126.8,100.3,99.8,99.3,97.6,81.8,81.3,80.9,80.5,79.9,79.6,79.5,78.6,76.0$, $75.8,75.4,75.1,74.9,74.6,74.2,73.9,73.3,73.2,73.1,72.8,72.2,72.1,72.0,72.0,71.7,71.5$, 70.4, 68.8, 68.7, 66.4, 64.0, 62.3, 30.3, 29.7, 21.2, 18.1, 11.9. MALDI-MS for $\mathrm{C}_{139} \mathrm{H}_{157} \mathrm{~N}_{3} \mathrm{O}_{26} \mathrm{Si}$ $\mathrm{M}_{\text {cald: }}$ 2312.08, $\mathrm{M}_{\text {found }}: 2334.76(\mathrm{M}+\mathrm{Na})^{+}, 2350.74(\mathrm{M}+\mathrm{K})^{+}$

Triethylammonium (2-O-Acetyl-3,4,6-O-tri-benzyl- $\alpha$-D-mannopyranosyl-(1 $\rightarrow 2)-3,4-O-d i$ -

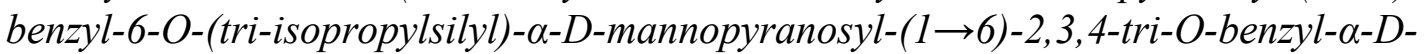

mannopyranosyl-( $1 \rightarrow 4)$-2-azido-2-deoxy-3, 6-O-benzyl- $\alpha$-D-glucopyranosyl-( $1 \rightarrow 6)$-2,3,4,5tetra-O-benzyl-1-O-(6-(benzylthio)hexyl-phosphonato)-D-myo-inositol (27) 

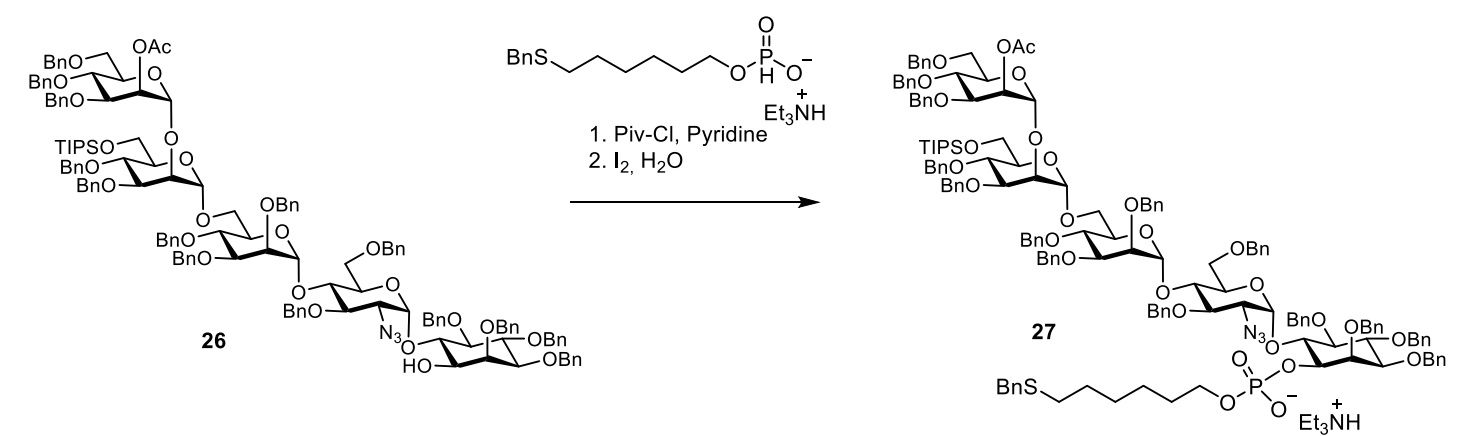

The pseudo-pentasaccharide alcohol $26(58 \mathrm{mg}, 0.025 \mathrm{mmol})$ and 6-(benzylthio)hexyl phosphonate ( $40 \mathrm{mg}, 0.103 \mathrm{mmol}$ ) were dissolved in and co-evaporated three times with $5 \mathrm{~mL}$ of pyridine and dried in HV overnight. The remaining was dissolved in dry pyridine $(3 \mathrm{ml})$ and pivaloyl chloride $(0.013 \mathrm{ml}, 0.103 \mathrm{mmol})$ was added under stirring and argon atmosphere. The reaction was stirred up to disappearance of the starting material by TLC (CH/AcOEt; $1.5: 1) 14$ h. $\mathrm{I}_{2}(6.45 \mathrm{mg}, 0.025 \mathrm{mmol})$ and water $(100 \mu \mathrm{L})$ were added and the reaction was stirred for 1 h. The mixture was diluted with $30 \mathrm{~mL}$ of DCM, extracted with aqueous sat. $\mathrm{Na}_{2} \mathrm{~S}_{2} \mathrm{O}_{3}$ and water, dried with $\mathrm{MgSO}_{4}$ and evaporated to dryness. The product was purified on a silica gel column deactivated with trimethylamine using $\mathrm{DCM} / \mathrm{MeOH}$ from 50:1 to 20:1 as eluent. The fractions containing the product were collected and evaporated to deliver $62 \mathrm{mg}(0.023 \mathrm{mmol}, 92 \%)$ of the phosphorylated pseudo-pentasaccharide 27. $\mathrm{R} f=0.24(\mathrm{DCM} / \mathrm{MeOH}, 10: 1),{ }^{1} \mathrm{H}$ NMR (400 $\left.\mathrm{MHz}, \mathrm{CDCl}_{3}-d\right) \delta 12.09(\mathrm{~s}, 1 \mathrm{H}), 7.80(\mathrm{~d}, J=7.8 \mathrm{~Hz}, 1 \mathrm{H}), 7.74-7.58(\mathrm{~m}, 4 \mathrm{H}), 7.22$ (tdt, $J=$ 30.5, 15.7, 6.7 Hz, 120H), $5.87(\mathrm{~s}, 1 \mathrm{H}), 5.37(\mathrm{~s}, 1 \mathrm{H}), 5.29(\mathrm{~s}, 1 \mathrm{H}), 5.18(\mathrm{~s}, 1 \mathrm{H}), 5.11(\mathrm{~s}, 1 \mathrm{H})$, $5.04-4.27(\mathrm{~m}, 35 \mathrm{H}), 4.23(\mathrm{~d}, J=11.4 \mathrm{~Hz}, 2 \mathrm{H}), 4.18-3.98(\mathrm{~m}, 5 \mathrm{H}), 3.97-3.40(\mathrm{~m}, 25 \mathrm{H})$, $3.35(\mathrm{~d}, J=10.8 \mathrm{~Hz}, 4 \mathrm{H}), 3.23-3.09(\mathrm{~m}, 2 \mathrm{H}), 2.96(\mathrm{dt}, J=9.4,4.8 \mathrm{~Hz}, 6 \mathrm{H}), 2.57-2.22(\mathrm{~m}$, $6 \mathrm{H}), 1.91(\mathrm{~s}, 3 \mathrm{H}), 1.61-1.36(\mathrm{~m}, 5 \mathrm{H}), 1.25(\mathrm{~h}, J=7.1 \mathrm{~Hz}, 23 \mathrm{H}), 0.86(\mathrm{dt}, J=13.5,4.8 \mathrm{~Hz}$, $2 \mathrm{H}),{ }^{31} \mathrm{P}$ NMR (162 MHz, $\left.\mathrm{cdcl}_{3}\right) \delta-0.94$, HRMS ESI-MS M calcd: $2597.1544 \mathrm{C}_{152} \mathrm{H}_{175} \mathrm{~N}_{3} \mathrm{O}_{29} \mathrm{PSSi}$, M found: $2596.1443(\mathrm{M}-\mathrm{H})^{-}$.

Bistriethylammonium (2-O-Acetyl-3,4,6-O-tri-benzyl- $\alpha$-D-mannopyranosyl-(1 $\rightarrow 2)-3,4-O$-dibenzyl-6-O-(2-(N-benzyloxycarbonyl) aminoethylphosphonato)-- $\alpha-D$-mannopyranosyl- $(1 \rightarrow 6)$ 2,3,4-tri-O-benzyl- $\alpha$-D-mannopyranosyl-( $1 \rightarrow 4)$-2-azido-2-deoxy-3,6-O-benzyl- $\alpha$ - $D$ -

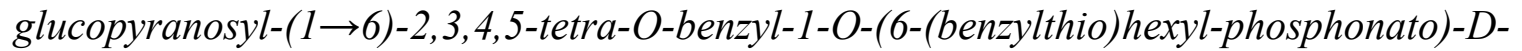
myo-inositol (29)
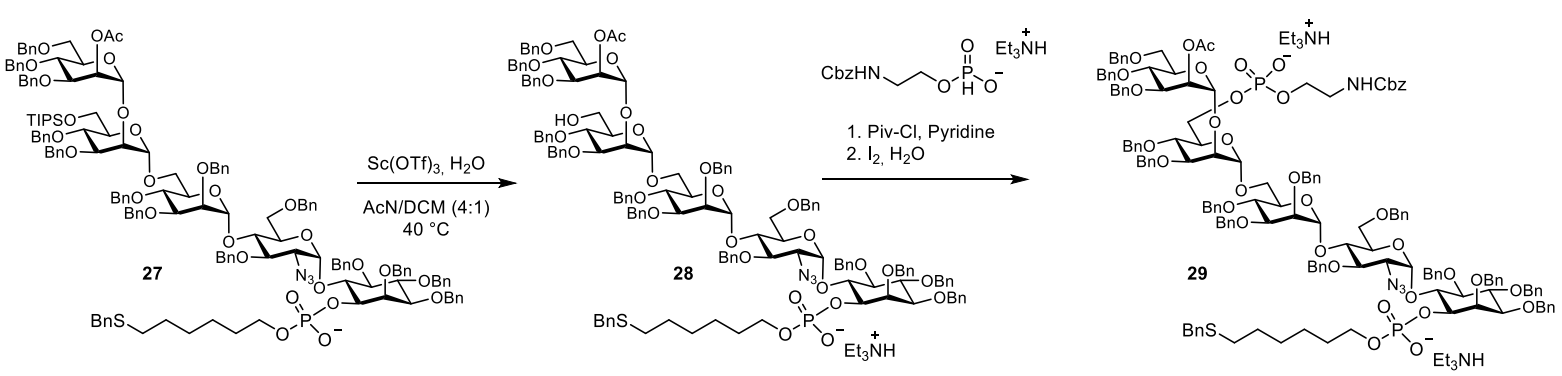

The pseudo-pentasaccharide $27(59 \mathrm{mg}, 0.021 \mathrm{mmol})$ were dissolved in $4 \mathrm{~mL}$ of an acetonitrile $/ \mathrm{CH}_{2} \mathrm{Cl}_{2}$ mixture (3:1). To this solution $\mathrm{Sc}(\mathrm{OTf})_{3}(41.4 \mathrm{mg}, 0.084 \mathrm{mmol})$ and water $(10 \mu \mathrm{l}, 0.555 \mathrm{mmol})$ were added. The mixture was stirred overnight at $40^{\circ} \mathrm{C}$. The reaction was quenched with $100 \mu \mathrm{L}$ of triethylamine, the solvent was evaporated and the residue was dried in vacuo. The product was passed through a Sephadex LH 20 column $(17 \times 700 \mathrm{~mm})$ and eluted with using a methanol $/ \mathrm{CH}_{2} \mathrm{Cl}_{2}$ (1:2) mixture containing $0.05 \%$ triethylamine. Collected fractions were evaporated and to obtain the crude product $\mathbf{2 8}$, which was directly used in the next reaction. The monophosphorylated alcohol $\mathbf{2 8}$ was dissolved in $3 \mathrm{~mL}$ of pyridine and the H-phosphonate 2-3 (36 mg, 0,100 mmol) was added. The pyridine was removed and the residue 
was co-evaporated three times with $5 \mathrm{~mL}$ of pyridine and dried in $\mathrm{HV}$ overnight. The reaction mixture was dissolved in anhydrous pyridine $(3 \mathrm{~mL})$ and PivCl $(4.69 \mu \mathrm{l}, 0.038 \mathrm{mmol})$ was added under stirring and argon atmosphere. The reaction was stirred up to disappearance of the starting material by TLC (CH/AcOEt; $1.5: 1) 14 \mathrm{~h}$. Iodine $(5.42 \mathrm{mg}, 0.021 \mathrm{mmol})$ and water $(100 \mu \mathrm{L})$ were added to the reaction mixture and stirred for $1 \mathrm{~h}$. The mixture was diluted with $30 \mathrm{~mL}$ of DCM and extracted with aq. saturated $\mathrm{Na}_{2} \mathrm{~S}_{2} \mathrm{O} 3$ and brine, dried with $\mathrm{MgSO} 4$ and evaporated to dryness. The crude product was purified on a silica gel column deactivated with triethylamine using DCM/MeOH from 50:1 to 20:1 as eluent to give the bis-phosphorylated product 29 in $88 \%$ (46 mg,0.015 mmol). $\mathrm{R} f=0.41\left(\mathrm{CH}_{2} \mathrm{Cl}_{2} / \mathrm{MeOH}, 10: 1\right),{ }^{1} \mathrm{H}$ NMR $(400 \mathrm{MHz}$, Chloroform- $d$ ) $\delta 12.32(\mathrm{~s}, 3 \mathrm{H}), 7.32(\mathrm{~d}, J=7.5 \mathrm{~Hz}, 2 \mathrm{H}), 7.26(\mathrm{~d}, J=7.7 \mathrm{~Hz}, 3 \mathrm{H}), 7.24-7.07$ $(\mathrm{m}, 65 \mathrm{H}), 7.05(\mathrm{dd}, J=13.3,6.4 \mathrm{~Hz}, 14 \mathrm{H}), 6.64(\mathrm{~d}, J=4.8 \mathrm{~Hz}, 1 \mathrm{H}), 5.89(\mathrm{~d}, J=3.7 \mathrm{~Hz}, 1 \mathrm{H})$, $5.45(\mathrm{~s}, 1 \mathrm{H}), 5.11(\mathrm{~s}, 1 \mathrm{H}), 4.97(\mathrm{dd}, J=12.2,8.1 \mathrm{~Hz}, 3 \mathrm{H}), 4.92-4.84(\mathrm{~m}, 3 \mathrm{H}), 4.80(\mathrm{ddd}, J=$ 17.6, 9.1, 3.5 Hz, 6H), $4.74(\mathrm{~s}, 3 \mathrm{H}), 4.73-4.63(\mathrm{~m}, 3 \mathrm{H}), 4.60-4.43(\mathrm{~m}, 6 \mathrm{H}), 4.41-4.29(\mathrm{~m}$, $9 \mathrm{H}), 4.25$ (dd, $J=13.9,7.6 \mathrm{~Hz}, 3 \mathrm{H}), 4.15(\mathrm{~d}, J=11.9 \mathrm{~Hz}, 2 \mathrm{H}), 4.08-3.62(\mathrm{~m}, 23 \mathrm{H}), 3.61-$ $3.38(\mathrm{~m}, 10 \mathrm{H}), 3.17(\mathrm{q}, J=4.9 \mathrm{~Hz}, 2 \mathrm{H}), 2.73(\mathrm{~s}, 16 \mathrm{H}), 2.28(\mathrm{t}, J=7.4 \mathrm{~Hz}, 2 \mathrm{H}), 2.01(\mathrm{~s}, 3 \mathrm{H})$, $1.51(\mathrm{p}, J=7.1 \mathrm{~Hz}, 3 \mathrm{H}), 1.43(\mathrm{p}, J=7.1 \mathrm{~Hz}, 2 \mathrm{H}), 1.32-1.10(\mathrm{~m}, 11 \mathrm{H}), 1.08-1.00(\mathrm{~m}, 26 \mathrm{H})$, $0.84-0.74(\mathrm{~m}, 3 \mathrm{H}) .{ }^{31} \mathrm{P}$ NMR $\left(162 \mathrm{MHz}, \mathrm{D}_{2} \mathrm{O}\right) \delta 0.3,0.2$. MALDI-MS for $\mathrm{C}_{153} \mathrm{H}_{166} \mathrm{~N}_{4} \mathrm{O}_{34} \mathrm{P}_{2} \mathrm{~S}^{-}$ $\mathrm{M}_{\text {calcd }}$ : 2698.0663, $\mathrm{M}_{\text {found: }}$ 2697.240.

$\alpha$-D-mannopyranosyl- $(1 \rightarrow 2)$ - 6-O-Aminoethylphosphonato- $\alpha$-D-mannopyranosyl- $(1 \rightarrow 6)$ $\alpha$-D-mannopyranosyl- $(1 \rightarrow 4)$-2-amino-2-deoxy- $\alpha$-D-glucopyranosyl- $(1 \rightarrow 6)-1-O$ -

(thiohexyl-phosphonato)-D-myo-inositol (5)

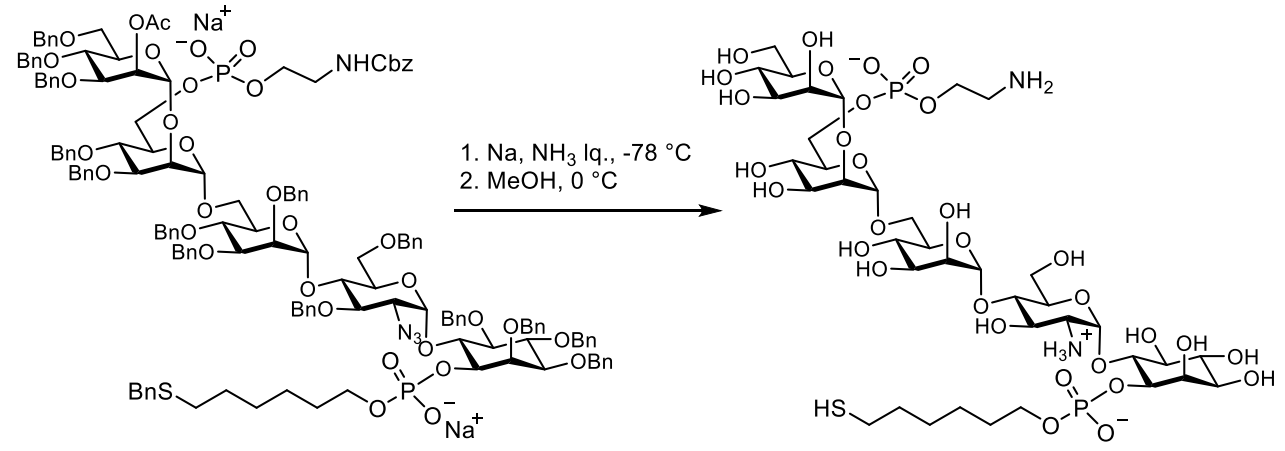

Ammonia $(10 \mathrm{~mL}, 693 \mathrm{mmol}))$ was condensed in a dried two-necked flask at $-78^{\circ} \mathrm{C}$. Sodium (50 mg, $2.175 \mathrm{mmol}$ ) was added until the solution was dark blue. The pseudo-pentasaccharide bisphosphate ( $20 \mathrm{mg}, 6.36 \mu \mathrm{mol})$ was dissolved in $4 \mathrm{~mL}$ of THF) and added via cannula, followed by $\mathrm{MeOH}(0.2 \mathrm{~mL})$. The resultant dark blue solution was stirred at $-78^{\circ} \mathrm{C}$ for $60 \mathrm{~min}$. Following disappearance of the blue color, methanol $(5 \mathrm{ml}, 124 \mathrm{mmol})$ was added to the mixture and ammonia was blown off with a stream of nitrogen. The reaction was warmed to room temperature and stirred for $1 \mathrm{~h}$. The reaction was quenched by addition of acetic acid, diluted with ethanol and evaporated to dryness. Chromatography of the crude mixture on Sephadex G25 (EtOH/H2O 1:20) gave the bis-phophorylated GPI-5 (5 mg, $4.37 \mu \mathrm{mol}, 77 \%$ yield). R $f=$ 0.30 (IPA/1M NH $4 \mathrm{OAc}, 2: 1),{ }^{1} \mathrm{H}$ NMR $(600 \mathrm{MHz}$, Deuterium Oxide) $\delta 5.42$ (d, $J=3.9 \mathrm{~Hz}$, $1 \mathrm{H}), 5.10(\mathrm{~s}, 1 \mathrm{H}), 5.02(\mathrm{~s}, 1 \mathrm{H}), 4.91(\mathrm{~s}, 1 \mathrm{H}), 4.11-4.03(\mathrm{~m}, 4 \mathrm{H}), 4.00(\mathrm{~d}, J=5.5 \mathrm{~Hz}, 5 \mathrm{H}), 3.95$ (s, 2H), $3.95-3.89(\mathrm{~m}, 3 \mathrm{H}), 3.85$ (dd, $J=13.9,8.3 \mathrm{~Hz}, 4 \mathrm{H}), 3.80$ (dd, $J=18.4,8.9 \mathrm{~Hz}, 4 \mathrm{H})$, $3.76-3.70(\mathrm{~m}, 6 \mathrm{H}), 3.69(\mathrm{~s}, 1 \mathrm{H}), 3.69-3.64(\mathrm{~m}, 5 \mathrm{H}), 3.60(\mathrm{dd}, J=19.4,9.9 \mathrm{~Hz}, 6 \mathrm{H}), 3.49(\mathrm{t}$, $J=10.0 \mathrm{~Hz}, 2 \mathrm{H}), 3.45-3.40(\mathrm{~m}, 1 \mathrm{H}), 3.32-3.24(\mathrm{~m}, 2 \mathrm{H}), 3.17(\mathrm{t}, J=5.0 \mathrm{~Hz}, 3 \mathrm{H}), 2.79(\mathrm{t}, J$ $=8.0 \mathrm{~Hz}, 0 \mathrm{H}), 2.65(\mathrm{t}, J=7.1 \mathrm{~Hz}, 2 \mathrm{H}), 2.25(\mathrm{t}, J=7.7 \mathrm{~Hz}, 1 \mathrm{H}), 1.60(\mathrm{dp}, J=14.3,7.3 \mathrm{~Hz}, 3 \mathrm{H})$, $1.56-1.50(\mathrm{~m}, 3 \mathrm{H}), 1.45(\mathrm{dd}, J=14.1,6.7 \mathrm{~Hz}, 1 \mathrm{H}), 1.36-1.13(\mathrm{~m}, 8 \mathrm{H}) .{ }^{13} \mathrm{C} \mathrm{NMR}(151 \mathrm{MHz}$, $\left.\mathrm{d}_{2} \mathrm{O}\right) \delta 97.9,104.6,101.1,104.9,78.8,73.6,74.1,67.1,64.4,72.7,81.2,72.6,68.9,69.2,80.0$, $63.7,72.9,62.7,74.8,68.9,74.3,72.7,73.0,75.9,79.0,68.8,63.7,74.8,69.5,73.2,75.4,56.6$, 
42.7, 53.6, 40.8, 63.4, 30.9, 32.3, 29.8, 27.2, 29.2. MALDI-MS for $\mathrm{C}_{38} \mathrm{H}_{71} \mathrm{~N}_{2} \mathrm{O}_{31} \mathrm{P}_{2} \mathrm{~S}^{-} \mathrm{M}_{\text {calcd: }}$ :

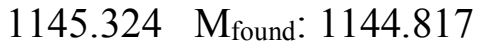

6-O-Aminoethylphosphonato-1-O-(thiohexyl-phosphonato)- $\beta$-D-galactopyranose (31)
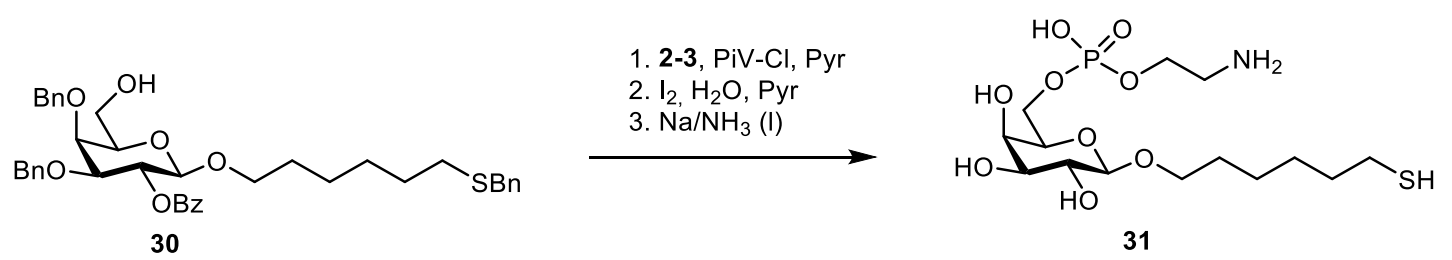

The galactosyl alcohol $\mathbf{3 0}^{6}(27 \mathrm{mg}, 0.040 \mathrm{mmol})$ and the H-phosphonate 2-3 (50 mg, 0.140 mmol) were dissolved in co-evaporated three times with $5 \mathrm{~mL}$ of pyridine and dried in vacuo. The reaction mixture was dissolved in anhydrous pyridine $(3 \mathrm{~mL})$, pivaloyl chloride $(17.2 \mu \mathrm{l}$, $0.4 \mathrm{mmol}$ ) was added and the reaction mixture was stirred for $4 \mathrm{~h}$ at room temperature. Iodine $(10.3 \mathrm{mg}, 0.040 \mathrm{mmol})$ and water $(50 \mu \mathrm{L})$ were added to the reaction and the mixture was stirred for $1 \mathrm{~h}$. The mixture was diluted with $10 \mathrm{~mL}$ of DCM and extracted with aq. saturated $\mathrm{Na}_{2} \mathrm{~S}_{2} \mathrm{O} 3$ and brine, dried with $\mathrm{MgSO} 4$ and evaporated to dryness. The product was purified on a silica gel column using DCM/MeOH (10:1) as eluent. Ammonia (10 mL, $693 \mathrm{mmol})$ ) was condensed in a dried two-necked flask at $-78^{\circ} \mathrm{C}$. Sodium $(50 \mathrm{mg}, 2.175 \mathrm{mmol})$ was added until the solution was dark blue. The galactosyl phosphate was dissolved in $4 \mathrm{~mL}$ of THF and added via cannula, followed by $\mathrm{MeOH}(0.2 \mathrm{~mL})$. The resultant dark blue solution was stirred at $-78^{\circ} \mathrm{C}$ for $60 \mathrm{~min}$. Following disappearance of the blue color, methanol $(5 \mathrm{ml})$ was added to the mixture and ammonia was blown off with a stream of nitrogen. The reaction was warmed to room temperature and stirred for $1 \mathrm{~h}$ to remove the ester. The reaction was quenched by addition of acetic acid, diluted with ethanol and evaporated to dryness. Chromatography of the mixture on Sephadex G-25 using $5 \% \mathrm{EtOH}$ in water gave the galactose 31 as a mixture of the monomer and disulfide ( $5 \mathrm{mg}, 0.012 \mathrm{mmol}, 31 \%$ yield). ${ }^{1} \mathrm{H}$ NMR (400 MHz, Deuterium Oxide) $\delta 4.26$ $(\mathrm{dd}, J=8.1,3.7 \mathrm{~Hz}, 1 \mathrm{H}), 3.96(\mathrm{dd}, J=5.9,4.3 \mathrm{~Hz}, 1 \mathrm{H}), 3.97-3.82(\mathrm{~m}, 3 \mathrm{H}), 3.81(\mathrm{~d}, J=3.4$ $\mathrm{Hz}, 1 \mathrm{H}), 3.72(\mathrm{dq}, J=14.8,6.7 \mathrm{~Hz}, 4 \mathrm{H}), 3.57-3.46(\mathrm{~m}, 2 \mathrm{H}), 3.34(\mathrm{dd}, J=9.9,7.9 \mathrm{~Hz}, 1 \mathrm{H})$, 3.12 (q, $J=5.3 \mathrm{~Hz}, 3 \mathrm{H}), 3.04(\mathrm{q}, J=7.3 \mathrm{~Hz}, 1 \mathrm{H}), 2.80-2.71(\mathrm{~m}, 1 \mathrm{H}), 2.65-2.47(\mathrm{~m}, 3 \mathrm{H})$, $1.65-1.53(\mathrm{~m}, 1 \mathrm{H}), 1.49(\mathrm{dt}, J=14.0,6.5 \mathrm{~Hz}, 9 \mathrm{H}), 1.33-1.22(\mathrm{~m}, 11 \mathrm{H}), 1.12(\mathrm{td}, J=7.4,2.3$ $\mathrm{Hz}, 2 \mathrm{H}) .{ }^{13} \mathrm{C}$ NMR $\left(101 \mathrm{MHz}, \mathrm{D}_{2} \mathrm{O}\right) \delta 102.7,73.4,73.3,72.5,70.6,70.5,68.1,66.3,64.2,61.7$, 61.6, 58.1, 50.9, 46.5, 39.9, 39.9, 38.0, 29.4, 29.4, 28.5, 28.4, 28.1, 27.4, 27.2, 24.5, 23.8, 20.9, 8.1. HRMS ESI-MS, $\mathrm{M}_{\text {calcd }}$ for the reduced form: $419.1379\left(\mathrm{C}_{14} \mathrm{H}_{30} \mathrm{NO}_{9} \mathrm{PS}\right), \mathrm{M}_{\text {found: }} 420.1422$ $(\mathrm{M}+\mathrm{H})^{+}, \mathrm{M}_{\text {calcd }}$ for the oxidized form: 836.2601 $\left(\mathrm{C}_{28} \mathrm{H}_{58} \mathrm{~N}_{2} \mathrm{O}_{18} \mathrm{P}_{2} \mathrm{~S}_{2}\right), \mathrm{M}_{\text {found: }} 837.2716(\mathrm{M}+\mathrm{H})^{+}$.

\section{Methods of Biochemistry}

$\mathrm{CRM}_{197}$ was purchased from Pfènex Inc., 1M solution of resin bound TCEP was purchased from Thermo scientific and autoclaved sterile water was used for the conjugation.

\section{Conjugation of GPI fragments 1-6 to CRM 197}

$250 \mu 1$ of TCEP resin solution was centrifuged for 3 mins and excess of water was removed. TCEP resin was suspended in $0.1 \mathrm{M}$ sodium phosphate buffer $(\mathrm{pH} 8)(150 \mu \mathrm{L})$ and centrifuged for 3 mins. The buffer excess was removed and autoclaved water was added. GPI fragment (in $120 \mu \mathrm{L}$ water) was transferred to the TCEP solution and incubated for $1 \mathrm{~h}$ at rt. After $1 \mathrm{~h}$, TCEP resin is filtered off using syringe and washed with autoclaved water $(5 \times 50 \mu \mathrm{L})$. All the water fractions are combined lyophilized. The reduction of disulfide to thiol is followed by LC-MS. To a stirred solution of $\mathrm{CRM}_{197}(3 \mathrm{mg})$ in $0.1 \mathrm{M}$ sodium phosphate buffer ( $\mathrm{pH} 7.4$ ) was added a solution of SBAP $(1.58 \mathrm{mg})$ in DMF $(60 \mu \mathrm{L})$ at room temperature. The reaction mixture was stirred for $1 \mathrm{~h}$. After $1 \mathrm{~h}$, the solution was concentrated to $250 \mu \mathrm{L}$ volume using an Amicon ${ }^{\circledR}$ 
Ultra-4 Centrifugal Filter Unit (10 kDa cut off, Millipore) and washed with water $(4 \mathrm{x} 1 \mathrm{~mL})$ and once with $0.1 \mathrm{M}$ sodium phosphate buffer ( $\mathrm{pH} 8.0$ ). Finally, the activated $\mathrm{CRM}_{197}$ is concentrated to obtain around $100 \mu \mathrm{L}$ of solution. To the stirred solution of activated $\mathrm{CRM}_{197}$ solution was added the reduced glycan. The solution was stirred overnight at room temperature. The solution was concentrated to $250 \mu \mathrm{L}$ of volume using an Amicon ${ }^{\circledR}$ Ultra-4 Centrifugal Filter Unit (10 kDa cut off, Millipore) and washed with water $(4 \mathrm{x} 1 \mathrm{~mL})$ and once with $0.1 \mathrm{M}$ sodium phosphate buffer ( $\mathrm{pH} 8.0$ ) to obtain the CRM-GPI conjugate. cysteine $(0.94 \mathrm{mg}$ ) was added directly to that solution in the Centricon and incubated for $1 \mathrm{~h}$ at r.t. After $1 \mathrm{~h}$, the mixture was washed with water $(4 \mathrm{x} 1 \mathrm{~mL})$ re-buffered with PBS to obtain the desired CRM-GPI conjugate. MALDI-TOF mass spectroscopy and SDS-PAGE was done for all the intermediates and final samples.

\section{Characterization of conjugates}

$\mathrm{CRM}_{197}$-GPI conjugates were characterized by MALDI-TOF mass spectrometry and SDSPAGE gel electrophoresis using staining against carrier protein with Coomassie Brilliant Blue R250 (CBB) (Sigma-Aldrich, Munich, Germany, 6104-59-2) or used for western blotting. For gel electrophoresis, glycoconjugates were diluted 1:10 in sterile water; loading buffer was added, boiled for 5 minutes and loaded onto a 12\% polyacrylamide gel. Gel electrophoresis was performed at $150 \mathrm{~V}, 150 \mathrm{~mA}$ for 60 minutes. Western blot transfer was accomplished at $100 \mathrm{~V}$ and $35 \mathrm{~mA}$. The membrane was routinely tested for positive transfer with Ponceau S (SigmaAldrich, Munich, Germany, 6226-79-5), subsequently blocked for $2 \mathrm{~h}$ at RT with 5\% BSA in PBS-T. Biotinylated Concavalin-A (Vector Laboratories, Burlingame, California, B-1005) was diluted 1:500 in 1x PBS 5\% BSA $0.01 \mathrm{mM} \mathrm{Mn}^{2+} 0.1 \mathrm{mM} \mathrm{Ca}^{2+}$ and incubated $2 \mathrm{~h}$ shaking at RT. The membrane was subsequently washed and streptavidin HRP (BD Pharmingen, Heidelberg, Germany, 557630) was added 1:500 in 1x PBS 5\% BSA 0.01 mM Mn ${ }^{2+} 0.1 \mathrm{mM}$ $\mathrm{Ca}^{2+}$ and incubated $1 \mathrm{~h}$ shaking at RT. Finally, the membrane was washed again in $1 \mathrm{x}$ PBS $0.01 \mathrm{mM} \mathrm{Mn}^{2+} 0.1 \mathrm{mM} \mathrm{Ca}^{2+}$ and developed by enhanced luminol-based chemiluminescent according to manufacturer's instructions (Thermo Fisher Scientific, Darmstadt, Germany, 32109). Dual color precision protein standard (Bio-Rad Laboratories, Munich, Germany, 1610374) was used as protein standard. 


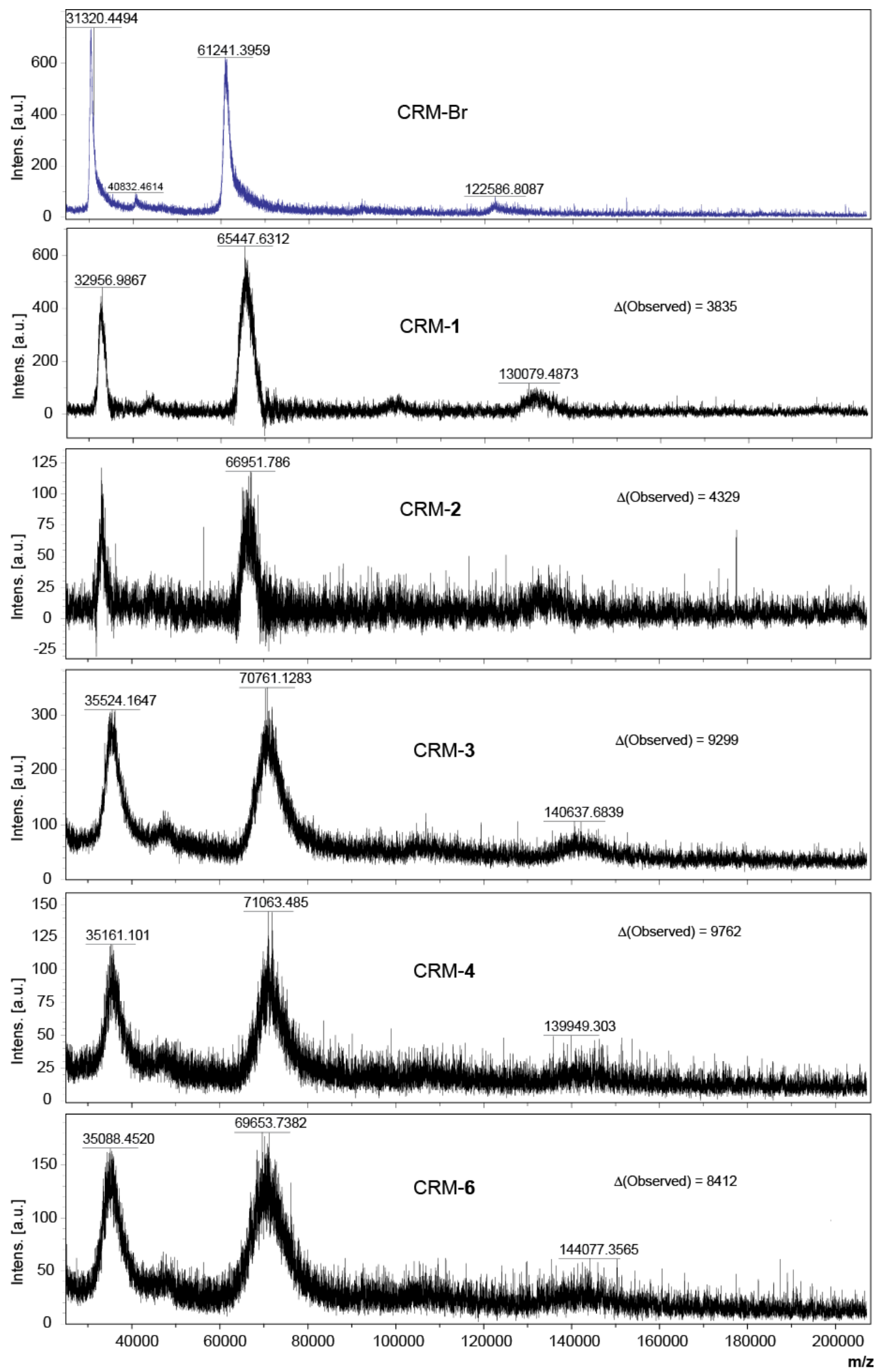

Figure S1: MALDI-TOF analysis of the activated CRM (CRM-AcBr) and CRM-GPI (CRMGlycan) during glycoconjugate synthesis. $\Delta$ (observed) represents the difference in $\mathrm{m} / \mathrm{z}$ for activated $\mathrm{CRM}_{197}$ and CRM-GPI and was used to calculate the glycan loading of the glycoconjugates. (A-E) represent the MALDI-TOF spectra for CRM 1-4 and 6 respectively. 

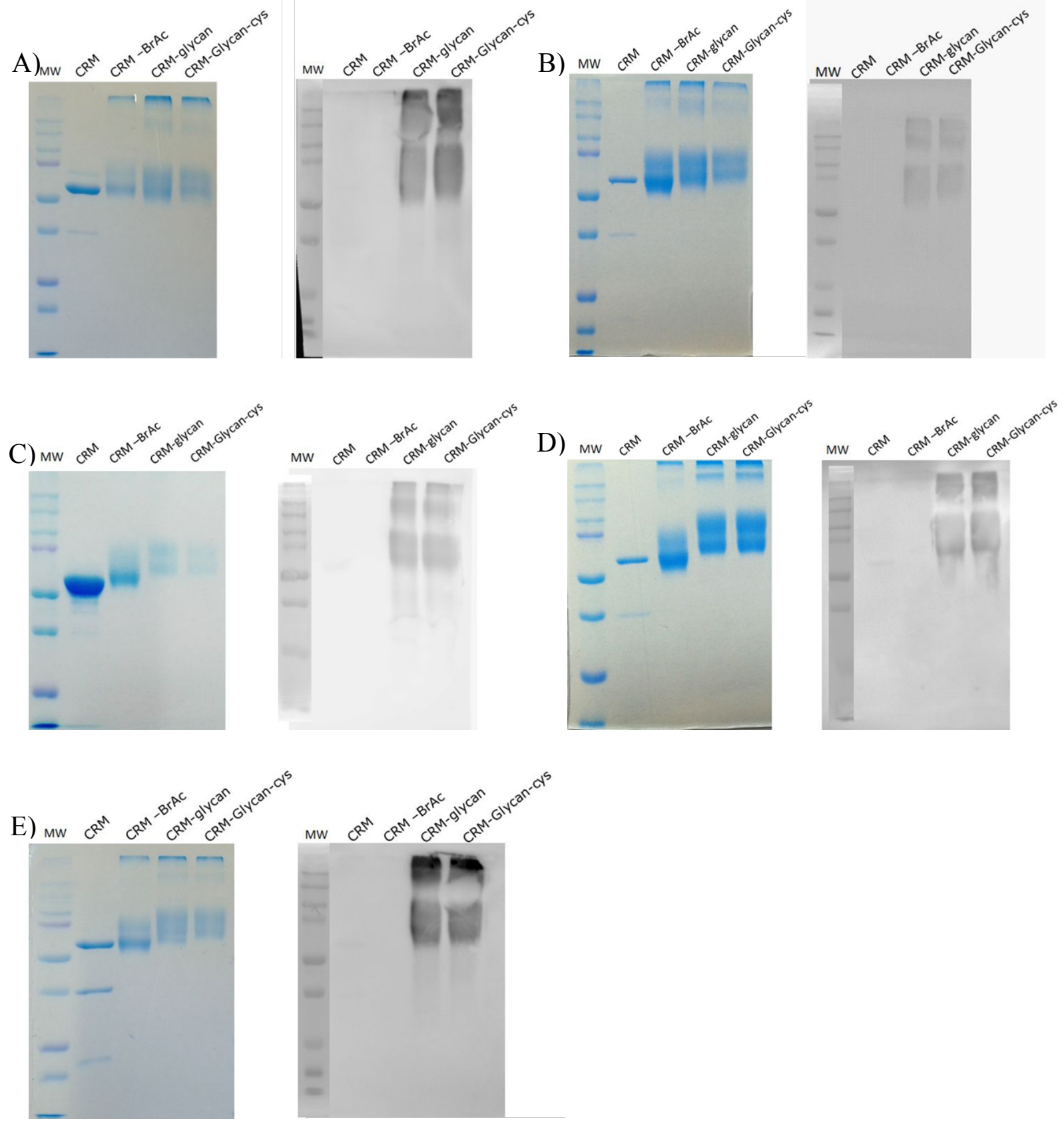

Figure S2: SDS PAGE and western blot analysis of the activated CRM (CRM-AcBr) and CRM-GPI (CRM-Glycan) and quenched glycoconjugate (CRM-glycan-cys) during glycoconjugate synthesis. (AE) represent the SDS PAGE and western blot for CRM 1-4 and 6 respectively. 


\begin{tabular}{|l|l|l|l|l|}
\hline $\begin{array}{l}\text { GPI- } \\
\text { CRM }\end{array}$ & Batch & $\Delta \mathrm{m} / \mathrm{z}$ & $\begin{array}{l}\text { GPI molecular } \\
\text { weight }\end{array}$ & $\begin{array}{l}\text { Loading: GPI/CRM } \\
\text { (\% of mass })\end{array}$ \\
\hline \multirow{2}{*}{ GPI1 } & 1 & 3835 & 781.82 & $4.92(6.5 \%$ glycan $)$ \\
\cline { 2 - 3 } & 2 & 4778 & & $6.12(7.2 \%$ glycan $)$ \\
\hline \multirow{2}{*}{ GPI2 } & 1 & 6972 & \multirow{2}{*}{904.87} & $7.4(10.2 \%$ glycan $)$ \\
\cline { 2 - 3 } & 2 & 4329 & & $5(7 \%$ glycan $)$ \\
\hline \multirow{2}{*}{ GPI3 } & 1 & 5668 & \multirow{2}{*}{943.96} & $5.7(8.1 \%$ glycan $)$ \\
\cline { 2 - 3 } & 2 & 9299 & & $9.8(13 \%$ glycan $)$ \\
\hline \multirow{2}{*}{ GPI4 } & 1 & 9865 & \multirow{2}{*}{1067.01} & $9.3(14 \%$ glycan $)$ \\
\cline { 2 - 3 } & 2 & 9792 & & $9.2(13.7 \%$ glycan $)$ \\
\hline \multirow{2}{*}{ GPI5 } & 1 & 5889 & \multirow{2}{*}{1146.99} & $5.2(8.9 \%$ glycan $)$ \\
\cline { 2 - 3 } & 2 & 10240 & & $8.9(14.3 \%$ glycan $)$ \\
\hline \multirow{2}{*}{ GPI6 } & 1 & 9322 & \multirow{2}{*}{1309.13} & $7.12(13 \%$ glycan $)$ \\
\cline { 2 - 3 } & 2 & 8412 & & $6.47(12 \%$ glycan $)$ \\
\hline
\end{tabular}

Figure S3: Glycan loading of the CRM glycoconjugates determined by MALDI-TOF mass spectrometry. Glycan loading was determined by MALDI and expressed as GPI molecules per CRM and as a percentage of weight for each CRM-GPI glycoconjugate, $\Delta \mathrm{m} / \mathrm{z}$ represents the difference in mass of the CRM-glycan and the activated CRM.

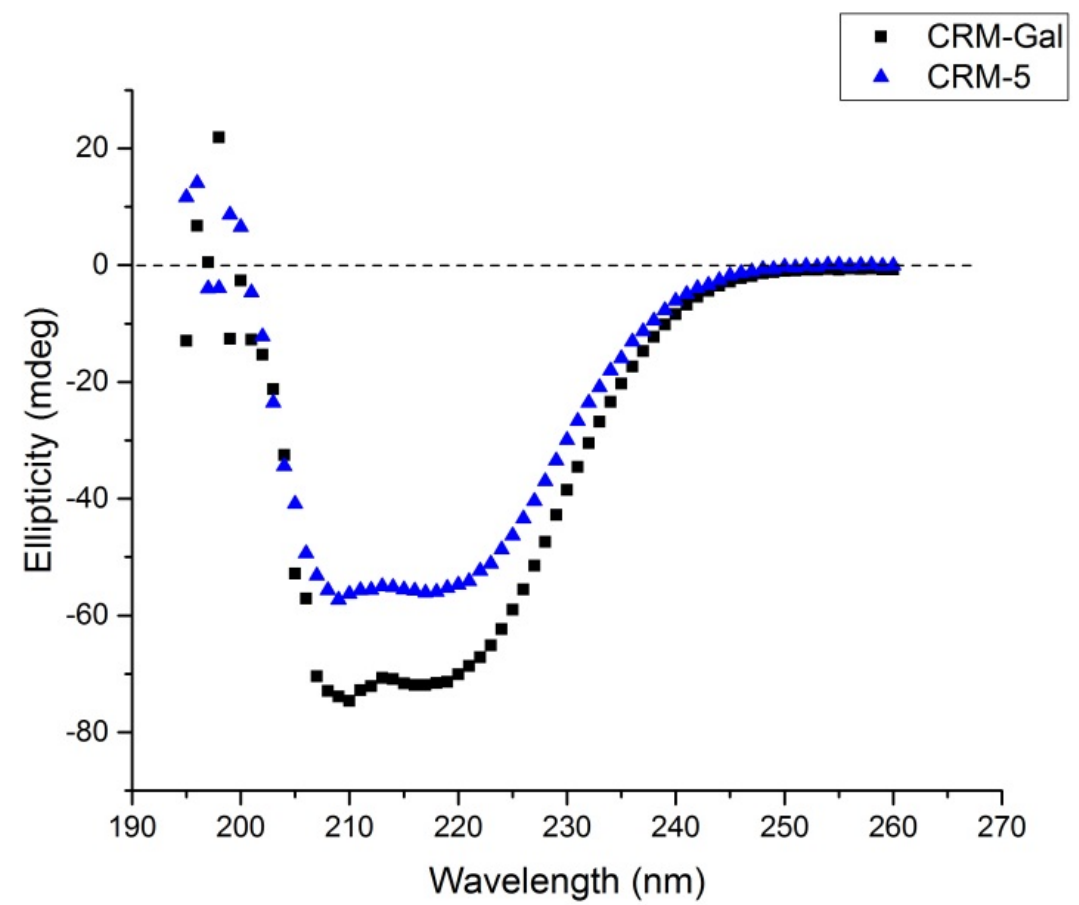

Figure S4. Circular dichroism analysis of CRM-5 and CRM-Gal.

\section{Animal Experiments}

Animals were treated strictly according to German (Tierschutz-Versuchstierverordnung) and European Law (Directive 2010/63/EU). Recommendations of the Society for Laboratory Animal 43 Science (GV-SOLAS) and of the Federation of European Laboratory Animal Science Associations (FELASA) were followed. The Office for Health and Social Affairs Berlin (LAGeSo) approved the experiment conclusively (Permit Number: G0239/14). All efforts were made to minimize suffering. All C57BL/6JRj mice used in this study were obtained 
from Janvier Labs (Saint-Berthevin, France). Mice were housed in individually-ventilated cages (IVCs) under specific pathogen free (SPF) conditions in the animal facility of the Federal Institute for Risk Assessment (BfR, Berlin, Germany). Mice were provided food and water ad libitum. Upon delivery (day -7), mice were allowed to rest for one week before experimental setting was started.

\section{Statistical analysis}

Statistical analysis was performed using the GraphPad Prism software (GraphPad Software Inc., La Jolla, CA, US). Unpaired Student's $t$ test was used to compare different sets of data, whereas two-way ANOVA was used to compare anti-GPI antibody levels over time between immunized and non-immunized groups. Log rank test was employed for analysis of survival between different groups. Statistical significance within figures is shown by asterisks: * represents $p<0.05, * * p<0.01, * * * p<0.001$ and $* * * * p<0.0001$.

\section{Immunization}

On day 0 , groups consisting of $15 \mathrm{C} 57 \mathrm{BL} / 6 \mathrm{JRj} 5$-week-old female mice were prime-immunized i.p. with CRM 1-6 or $\mathrm{CRM}_{197}-\mathrm{Gal}$ as a control. Two boost-immunizations were performed in 14 day intervals (day 14 and day 28). Each mouse was injected a total of $100 \mu \mathrm{L}$ i.p. of either GPI-CRM $_{197}$ or $\mathrm{CRM}_{197}$-Gal formulated with aluminum hydroxide (alum). Immunizations

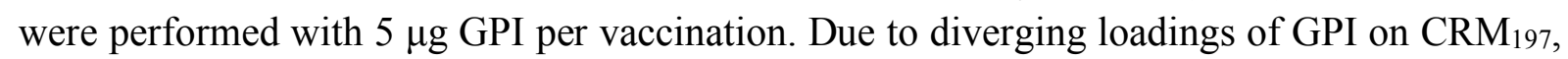

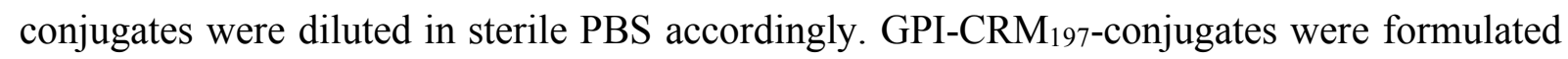
with aluminum hydroxide 1:2 (Alhydrogel ${ }^{\circledR}$, Brenntag, Denmark) and rotated over night at 4 ${ }^{\circ} \mathrm{C}$ before immunization. 
A)

$$
\mathrm{CRM}_{197^{-}} \text {GPI1- GPI2- GPI3- GPI4- GPI5- GPI6- Pos. }
$$

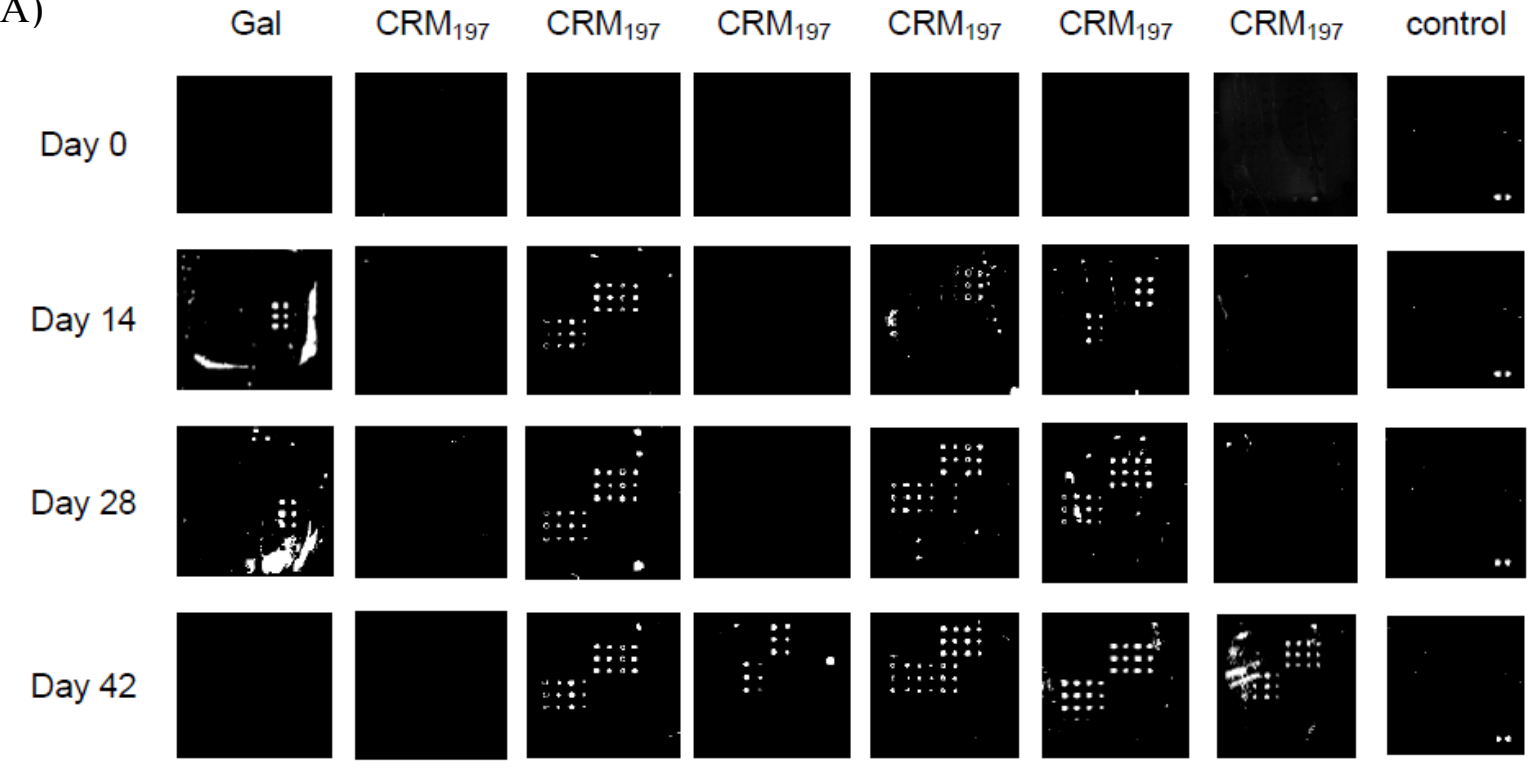

B)

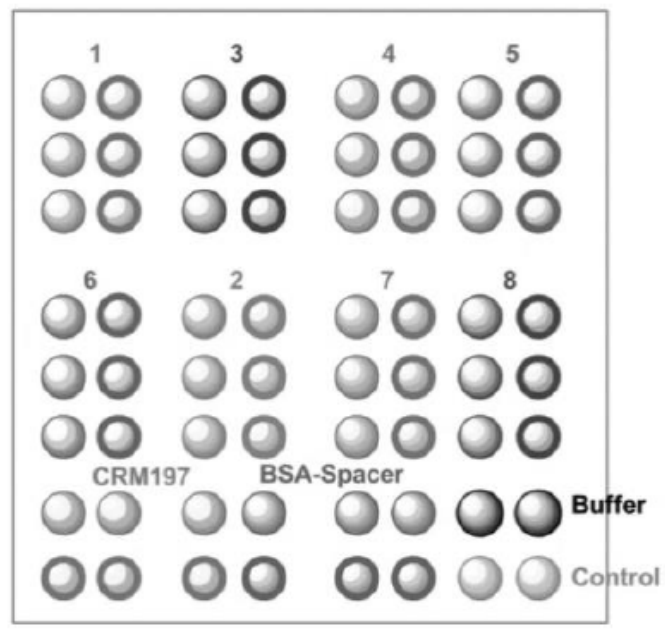

$2 \mathrm{GPI}-2$

$3 \mathrm{GPI}-3 \quad 7 \mathrm{GPI}$-control

4 GPI-4 8 Galactose

$1 \mathrm{mM}$ (glycan); $1 \mu \mathrm{M}$ (protein)

$0.2 \mathrm{mM}$ (glycan); $0.2 \mu \mathrm{M}$ (protein)

Figure S5: Glycan microarray for glycoconjugates at two concentrations for anti-GPI antibody level at days $0,14,28$ and 42. (A) Representative microarray wells results from serum of mice immunized with CRM-1-6 (day 0-42) and rabbit anti-S. pneumoniae antibodies as positive control. (B) Microarray printing pattern of maleimide treated glass slides (PolyAn, Berlin, Germany). 

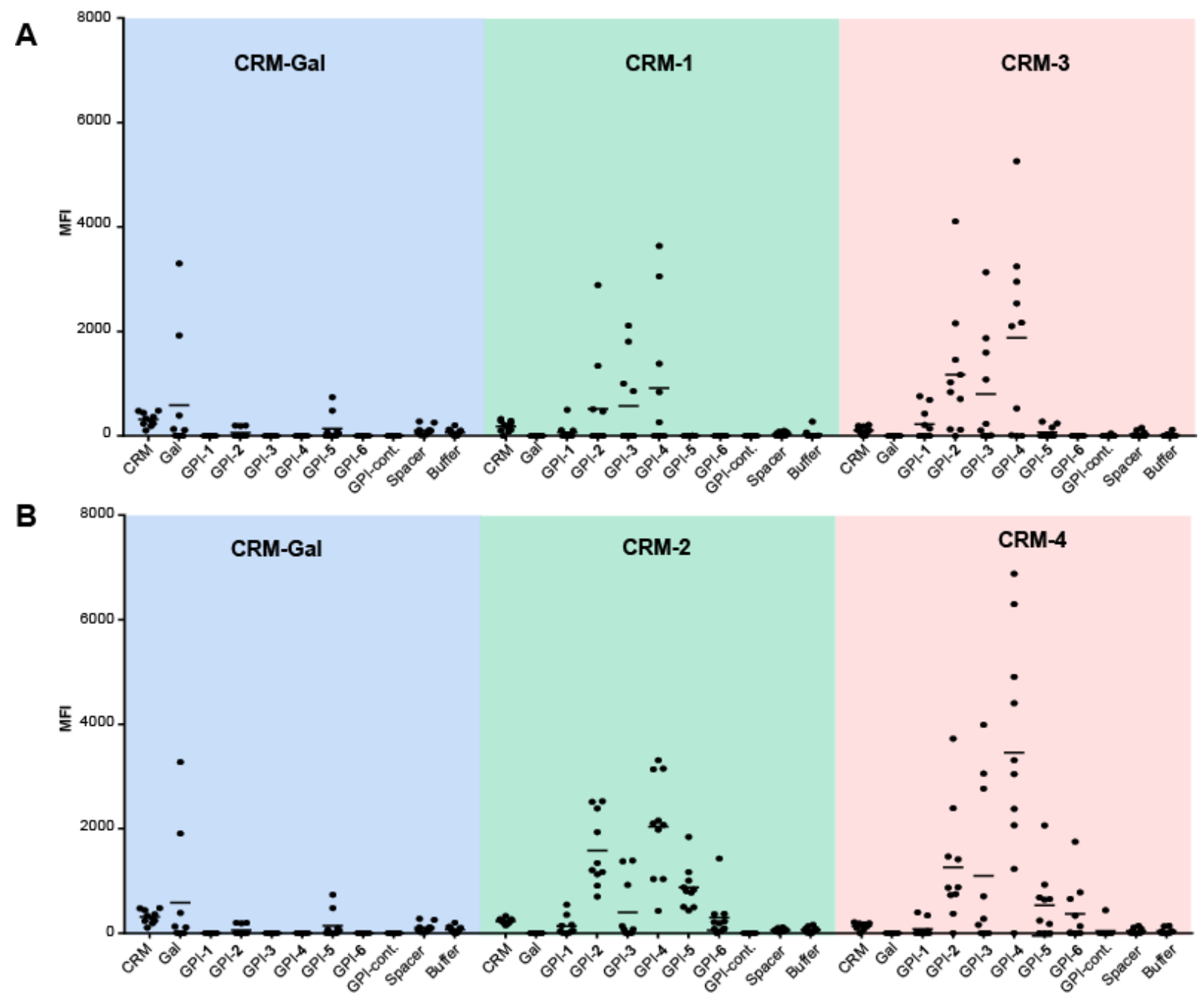

C

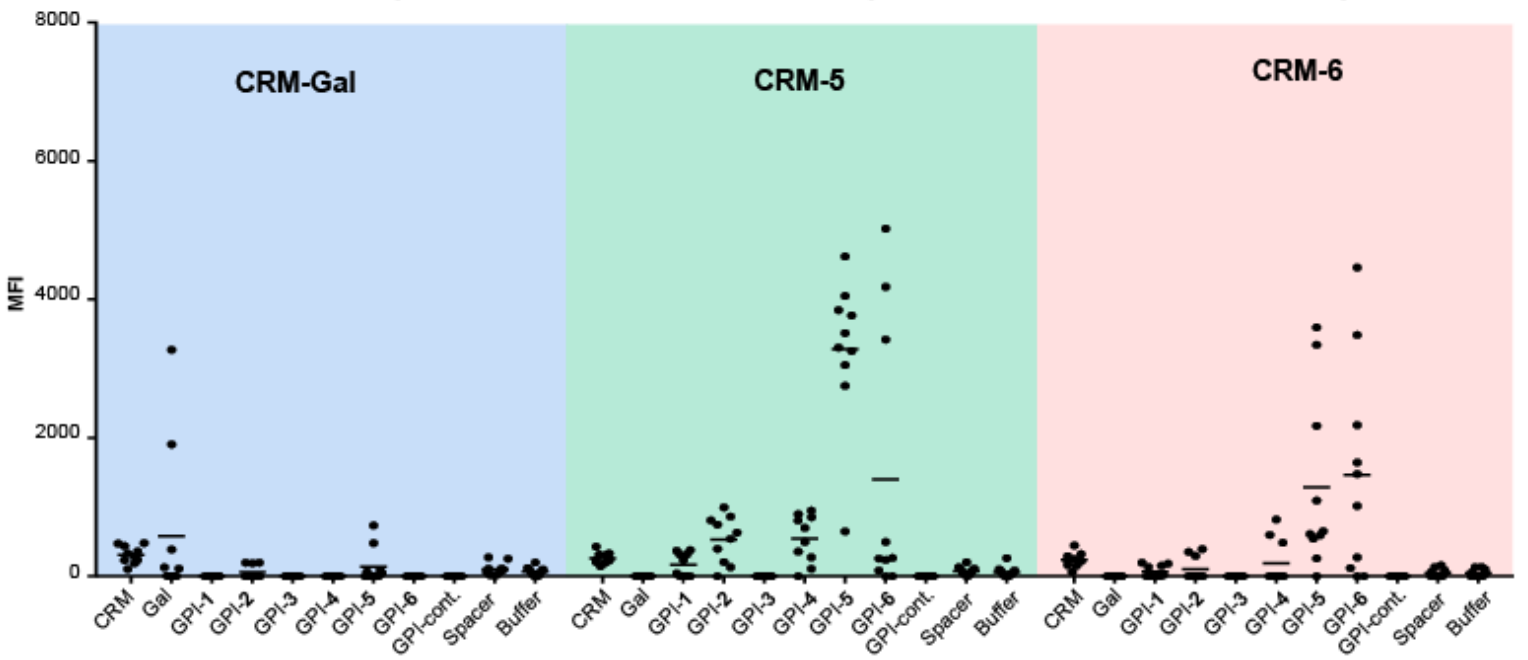

Figure S6: Cross-reactivity of anti-GPI antibodies after day 42. (A) Cross-reactivity of serum from CRM-Gal, CRM-1 and CRM-3 immunized mice against synthetic GPIs (B) Cross-reactivity of serum from CRM-Gal, CRM-2 and CRM-4 immunized mice against synthetic GPIs (C) Cross-reactivity of serum from CRM-Gal, CRM-5 and CRM-6 immunized mice against synthetic GPIs. 
A)

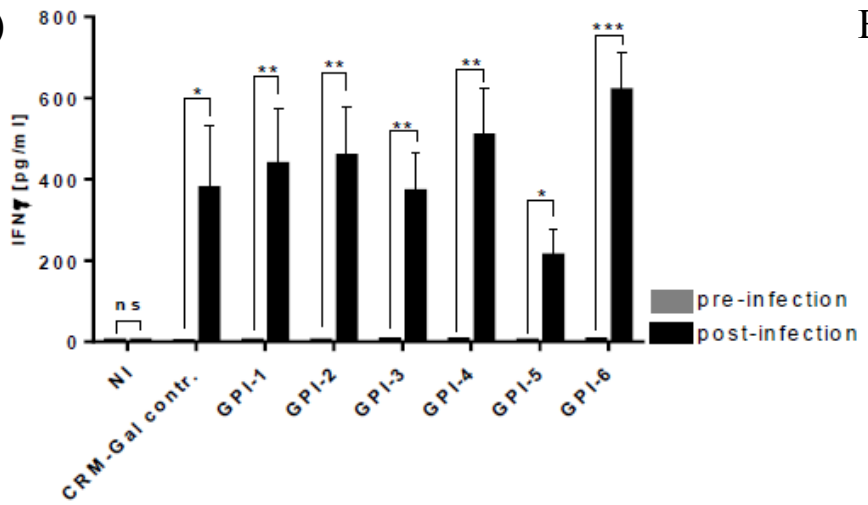

B)

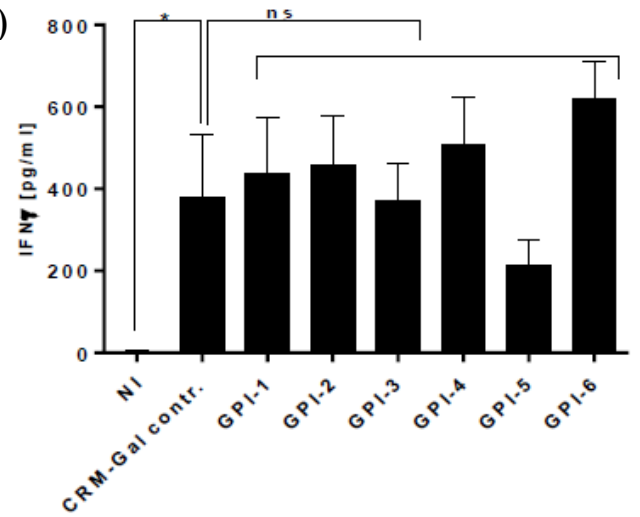

C)

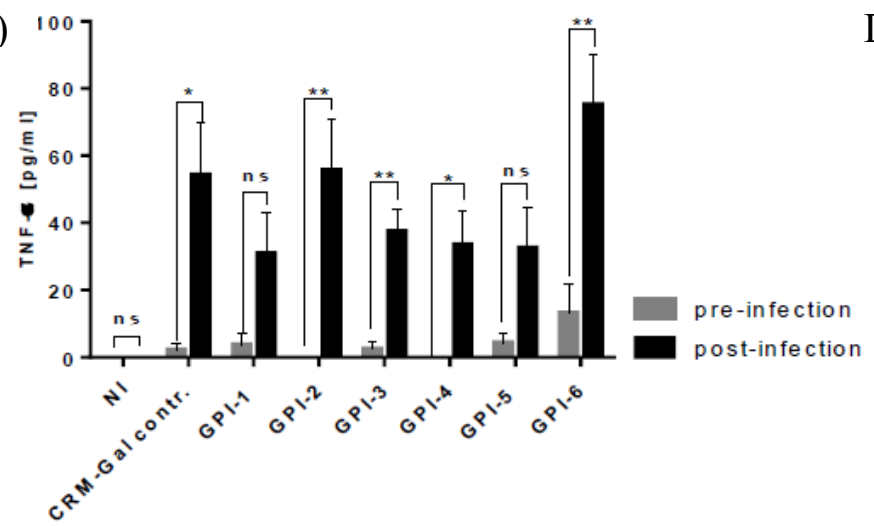

D)

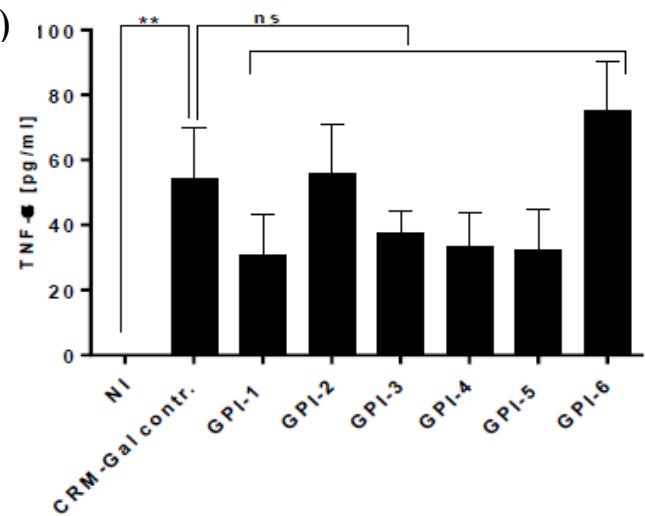

E)

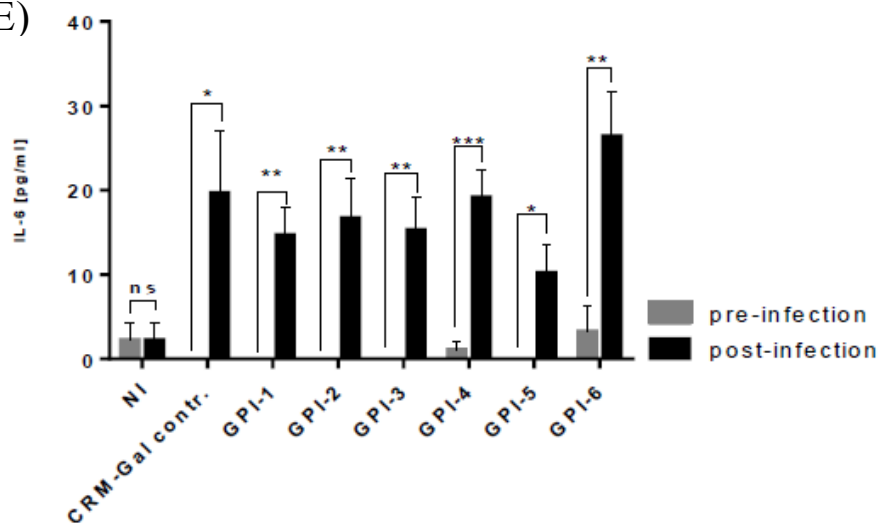

F)

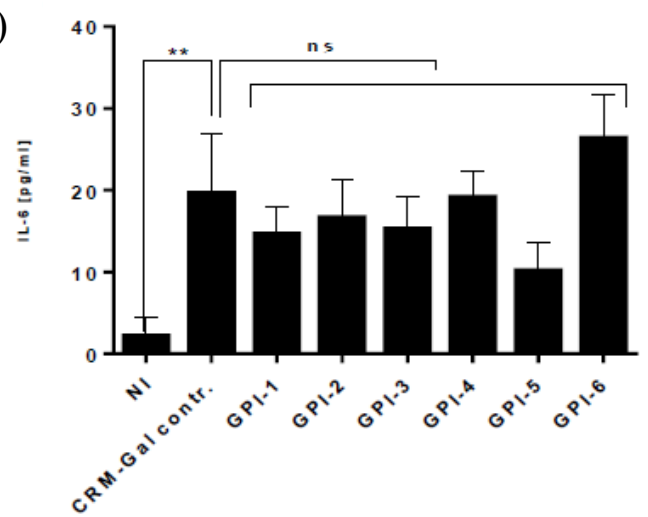

Figure S7: Pro-inflammatory cytokine levels of infected mice. (A, C, E) comparison in the level of IFN- $\gamma$, TNF- $\alpha$ and IL-6 for CRM 1-6 immunized and control mice before and after infection. (B, D, F) Comparison in the serum levels of pro-inflammatory cytokines between non-infected mice and CRMGPI vaccinated mice. Statistical significance was determined using the unpaired Student's t-test, significance shown by asterisks $*(\mathrm{p}<0.05), * *(\mathrm{p}<0.01), * * *(\mathrm{p}<0.001)$; not significant results labeled ns $(\mathrm{p}>0.05)$ 


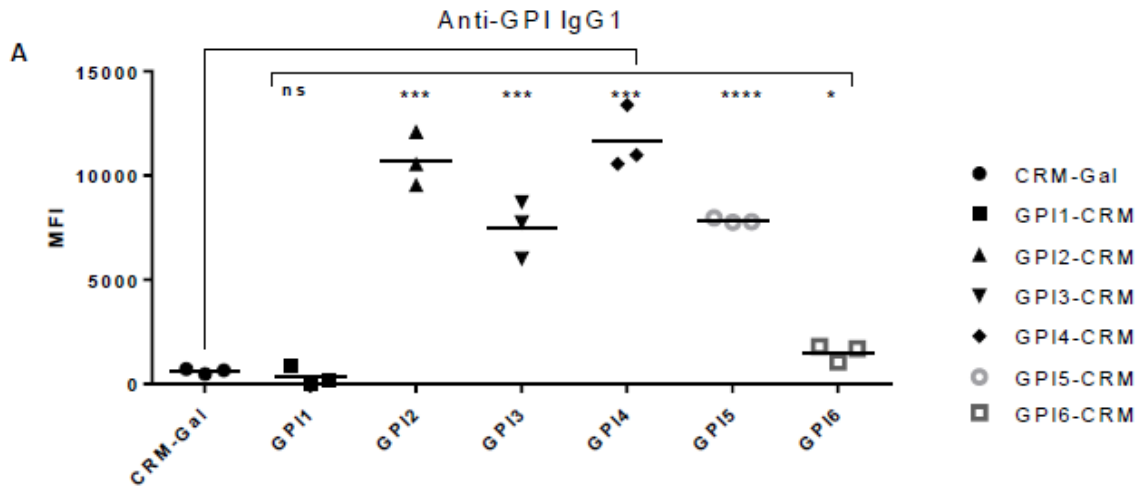

B
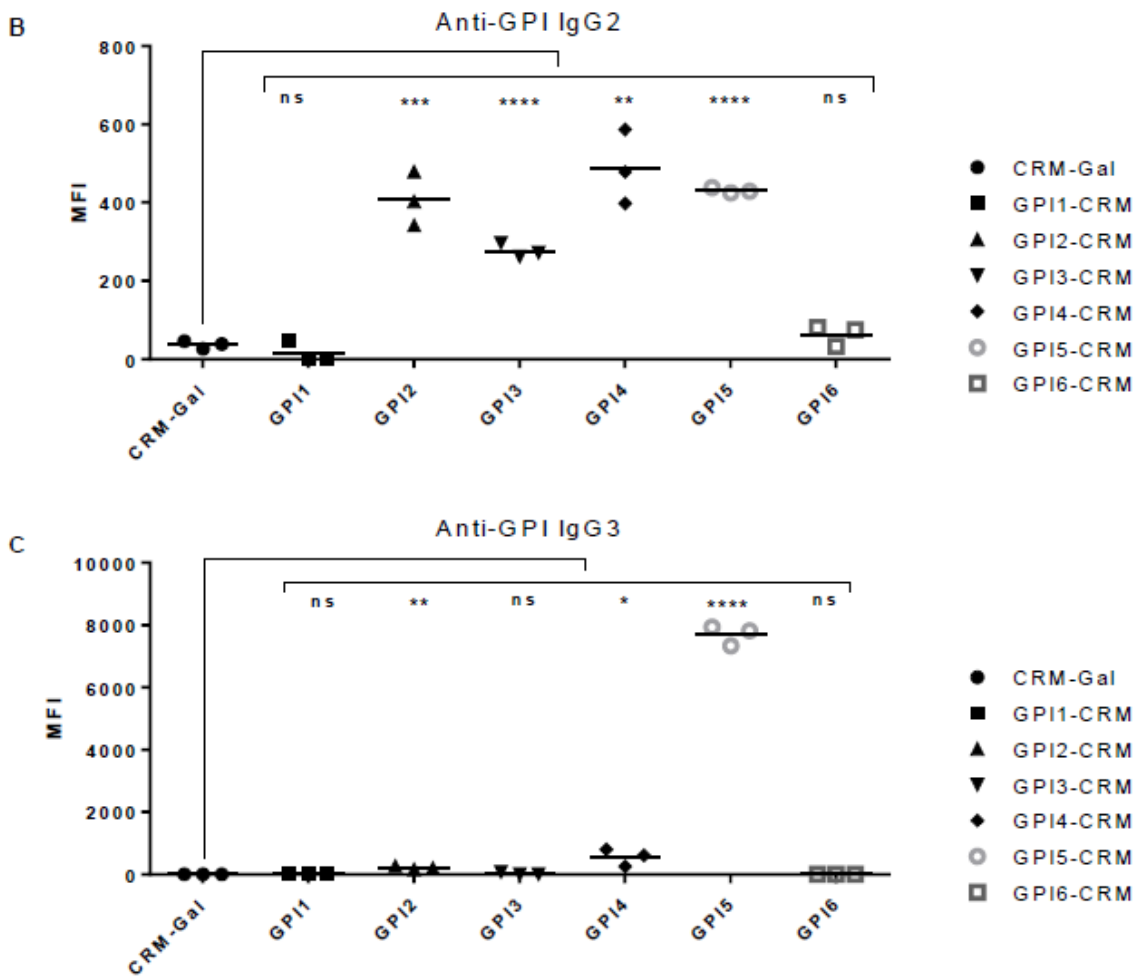

Figure S8. Endpoint anti-GPI antibody titer for IgG subclasses measured by glycan array for mice immunized with GPI-CRM-conjugates at day 42. Serum antibody levels at day 42 against CRM and synthetic GPI glycans 1-6 in mice immunized with CRM-Gal and CRM 1-6 for IgG subclasses IgG1 (A), $\operatorname{IgG} 2$ (B) and IgG3 (C). 


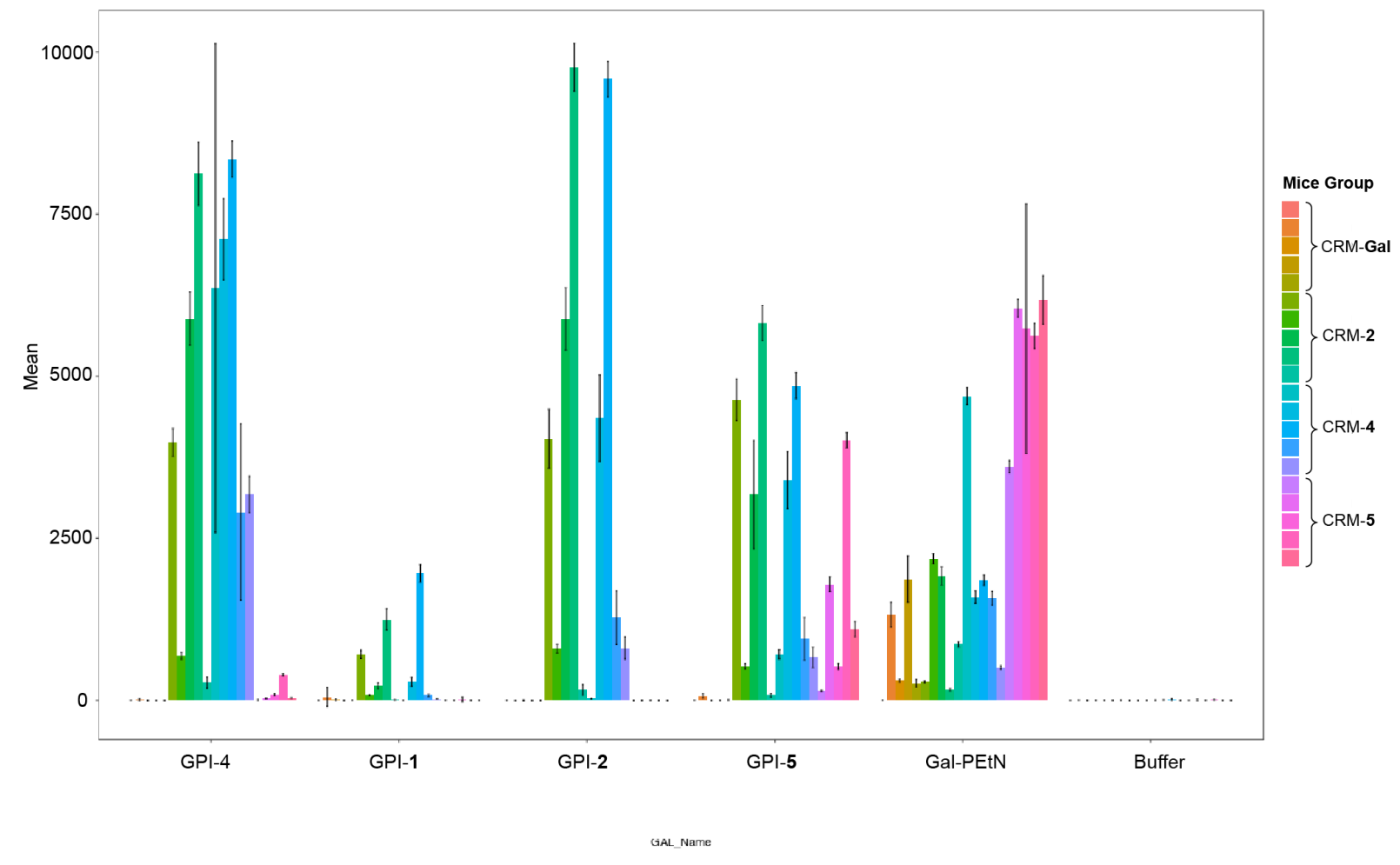

Figure S9. Binding of Antibodies to Gal-PEtN. Thea analysis was performed for the groups CRM-Gal, CRM-3, CRM- 

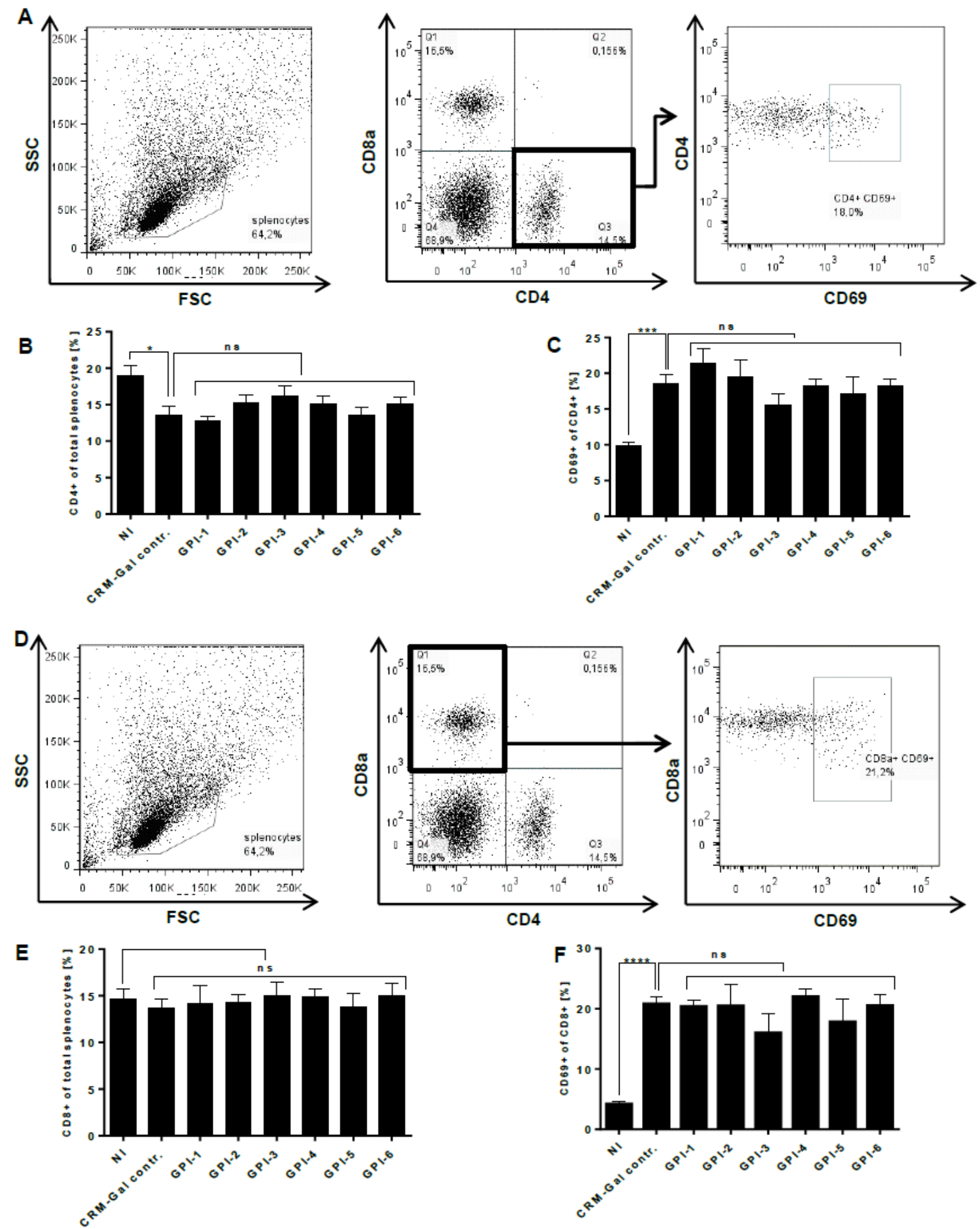

Figure S10. Spleen $\mathrm{CD}^{+}$and $\mathrm{CD} 4^{+} \mathrm{T}$ cell activation in immunized and P. berghei ANKA-challenged mice (A, D) Representative gatings of activated $\mathrm{CD} 4^{+}$and $\mathrm{CD}^{+}$spleen $\mathrm{T}$ cells. Spleen cells were isolated from immunized and $P$. berghei ANKA infected mice on day 6 post infection and quantified by flow cytometry. (B, E). (C, F) Cellular activation (measured by activation marker CD69) was marked in both $\mathrm{CD}^{+}$and $\mathrm{CD}^{+} \mathrm{T}$ cells compared to non-infected controls. Statistical significance was determined using Student's t-test, statistical significance shown by asterisks $*(p<0.05), * * *(p<0.001)$ and $* * * *(\mathrm{p}<0.0001)$. 


\section{NMR Spectra}

1-O-(6-thiobenzyl)hexyl-3,6-O-benzyl-2-deoxy-2-N-phthalimido- $\beta$-D-glucopyranoside (8)
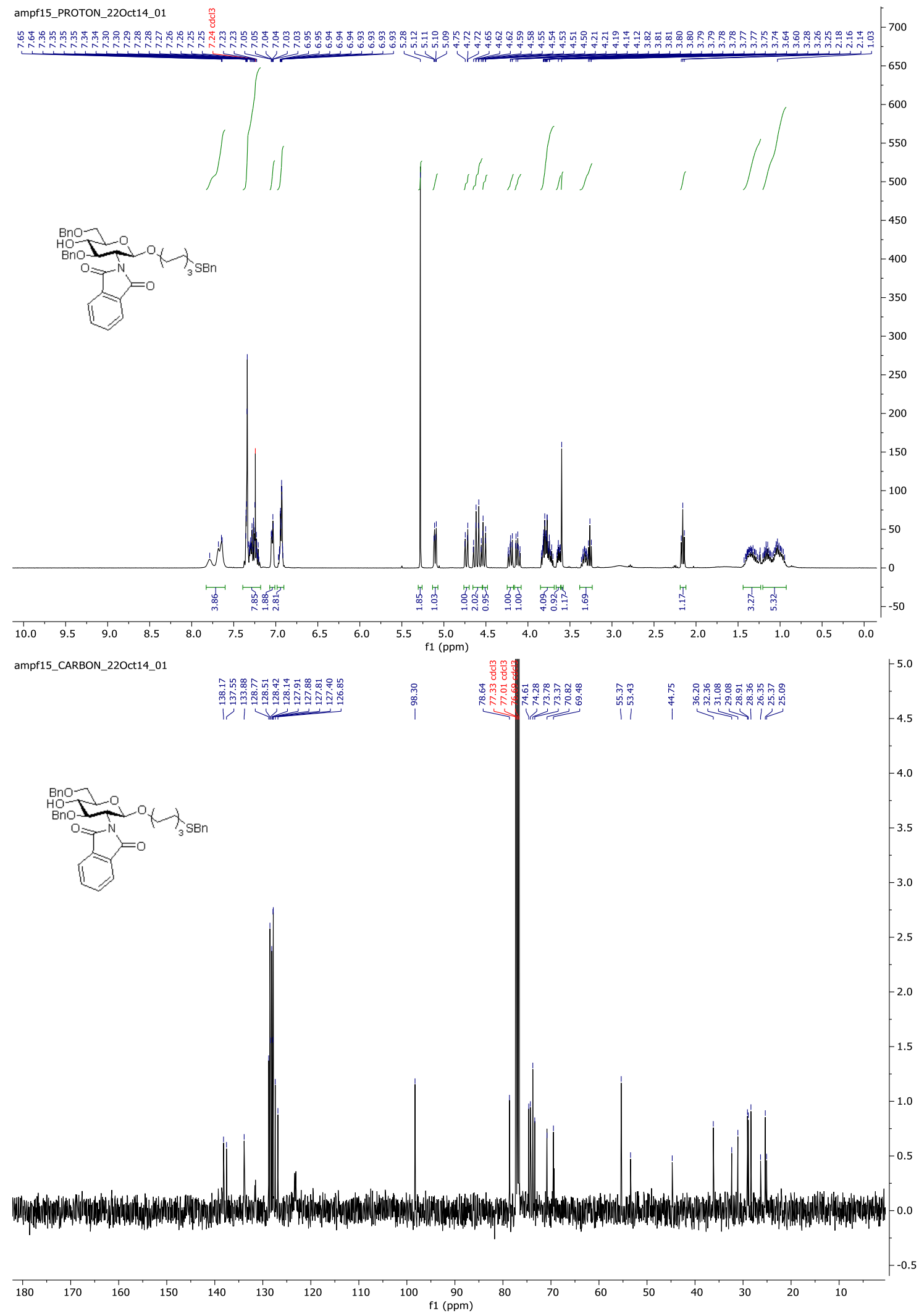

2-O-Acetyl-3,4-di-O-benzyl-6-O-triisopropylsilyl- $\alpha$-D-mannopyranosyl-(1 $\rightarrow 4)-1-O-(6-$ thiobenzyl)hexyl-3,6-O-benzyl-2-deoxy-2-N-phthalimido- $\beta$-D-glucopyranoside (9) 


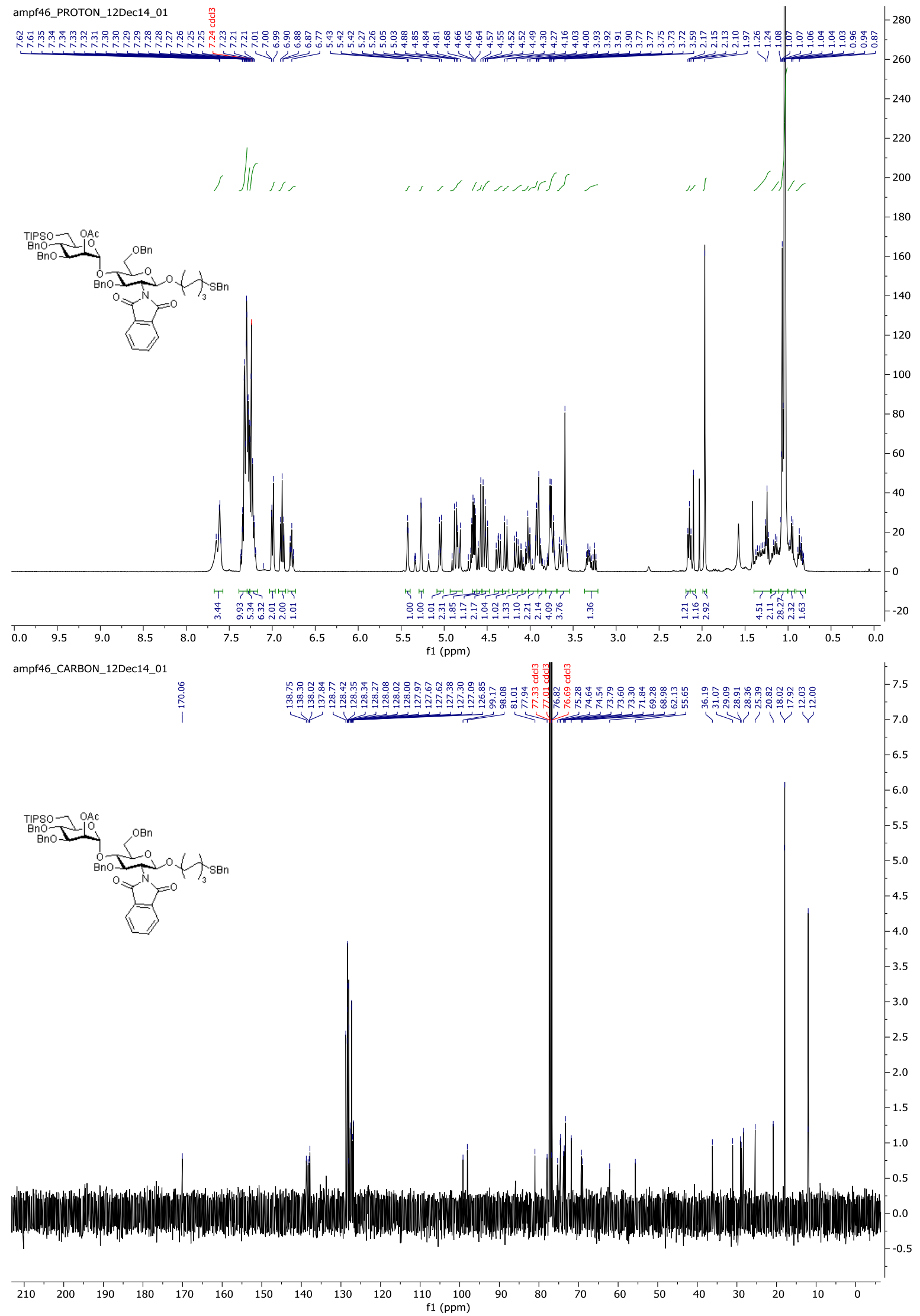

2-O-Acetyl-3,4-di-O-benzyl- $\alpha$-D-mannopyranosyl- $(1 \rightarrow 4)-\quad$ 1-O-(6-thiobenzyl)hexyl-3, 6-Obenzyl-2-deoxy-2-N-phthalimido- $\beta$-D-glucopyranoside (10) 


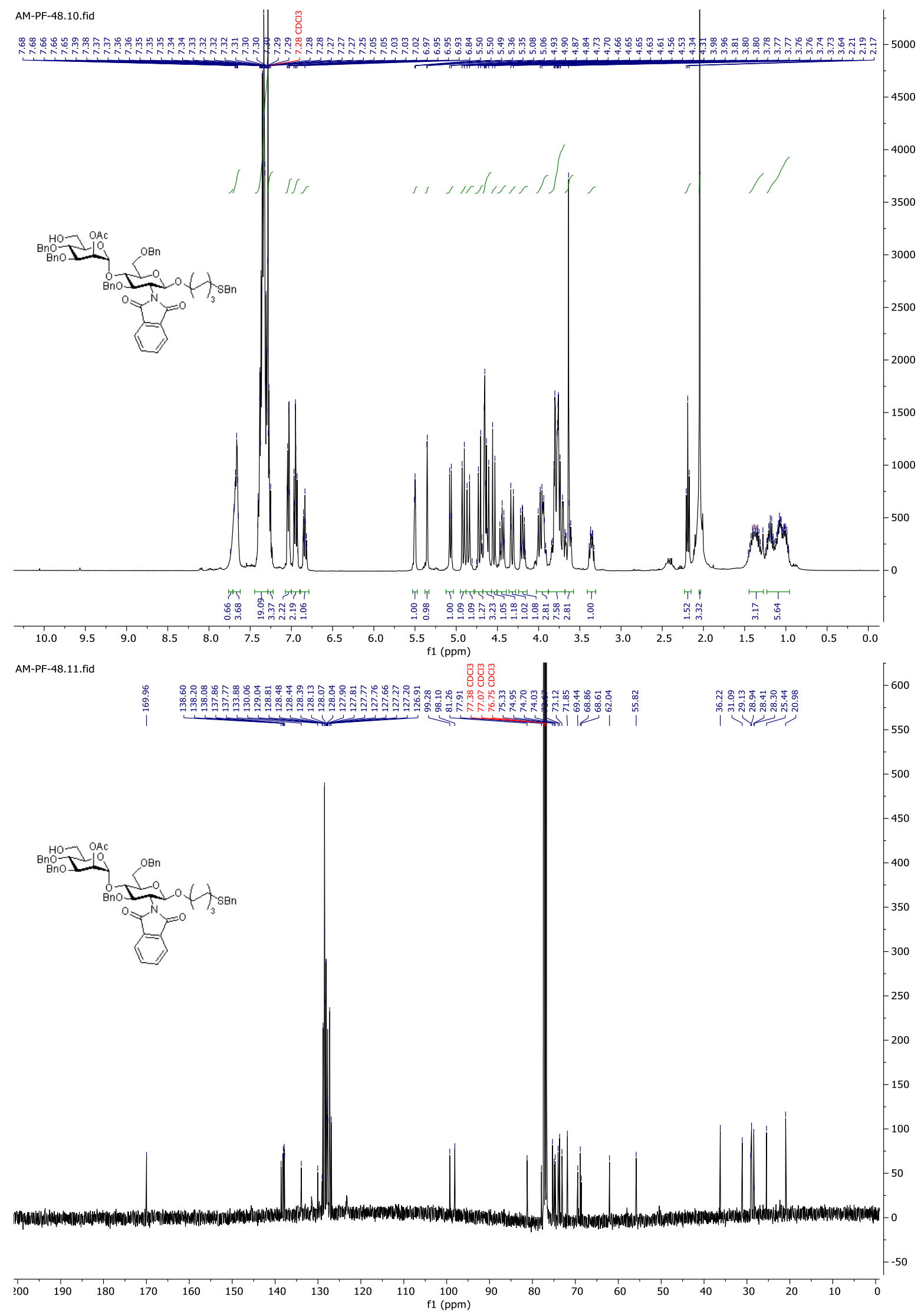

1-O-(6-thiobenzyl)hexyl-2-O-acetyl-3,4-di-O-benzyl-6-O-triisopropylsilyl- $\alpha$-D-

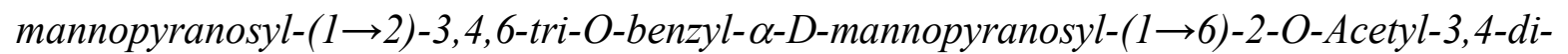
O-benzyl- $\alpha$-D-mannopyranosyl-( $1 \rightarrow 4)$-3,6-O-benzyl-2-deoxy-2-N-phthalimido- $\beta$ - $D$ glucopyranoside (13) 


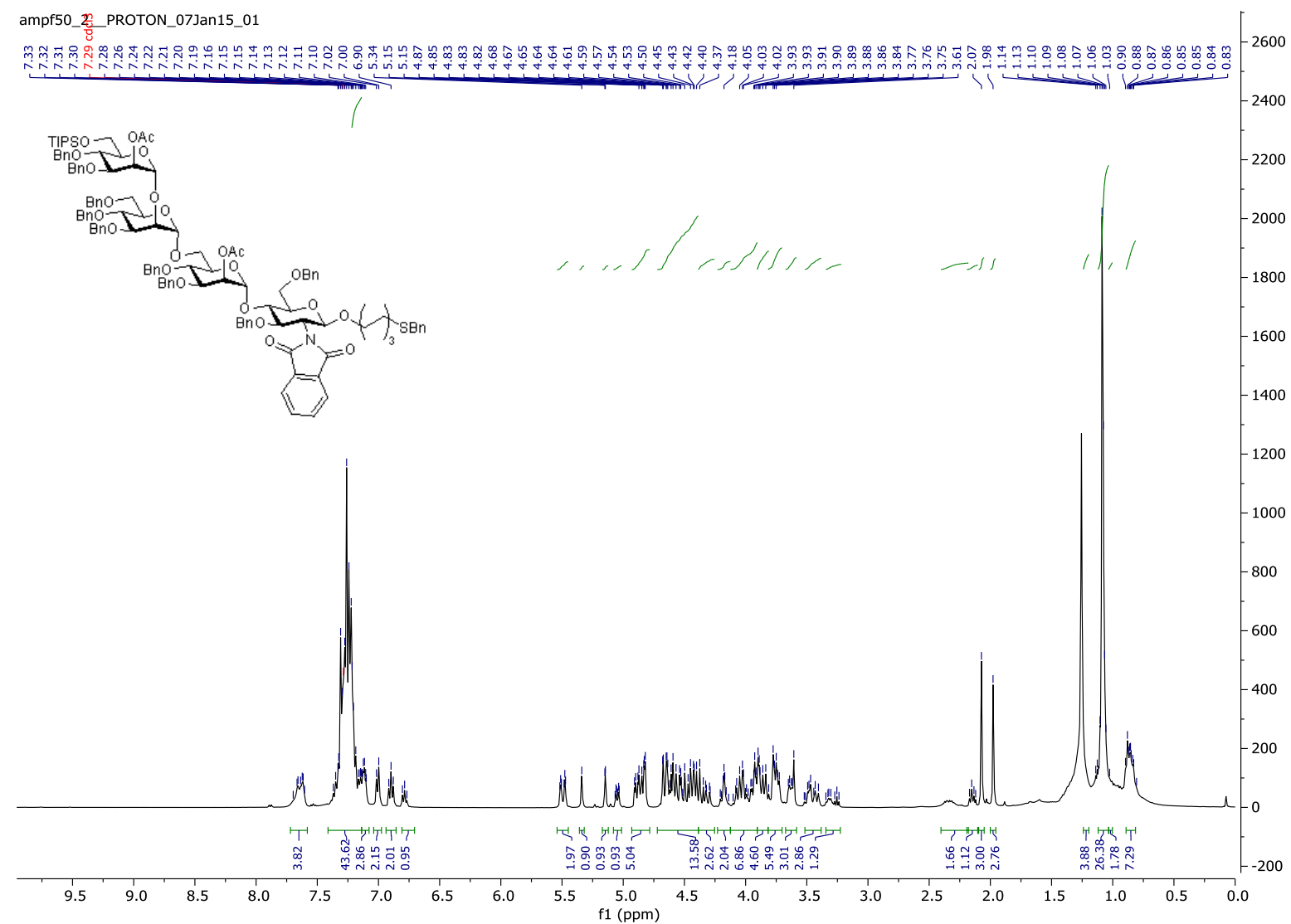




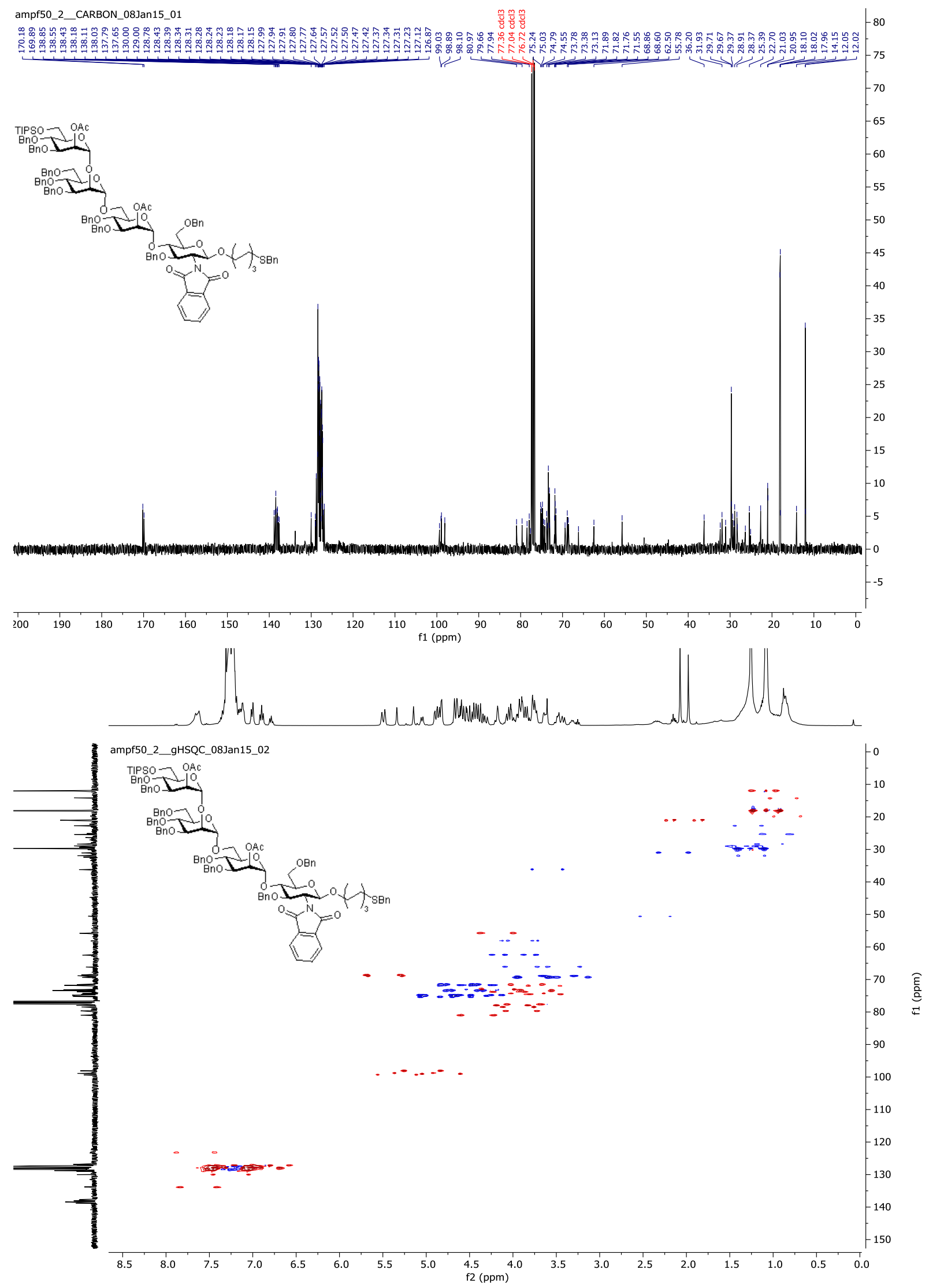


1-O-(6-thiobenzyl)hexyl-2-O-acetyl-3,4-di-O-benzyl- $\alpha$-D-mannopyranosyl-(1 $\rightarrow 2)-3,4,6$-tri-

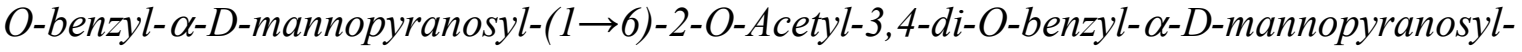
$(1 \rightarrow 4)$-3, 6-O-benzyl-2-deoxy-2-N-phthalimido- $\beta$-D-glucopyranoside (14)

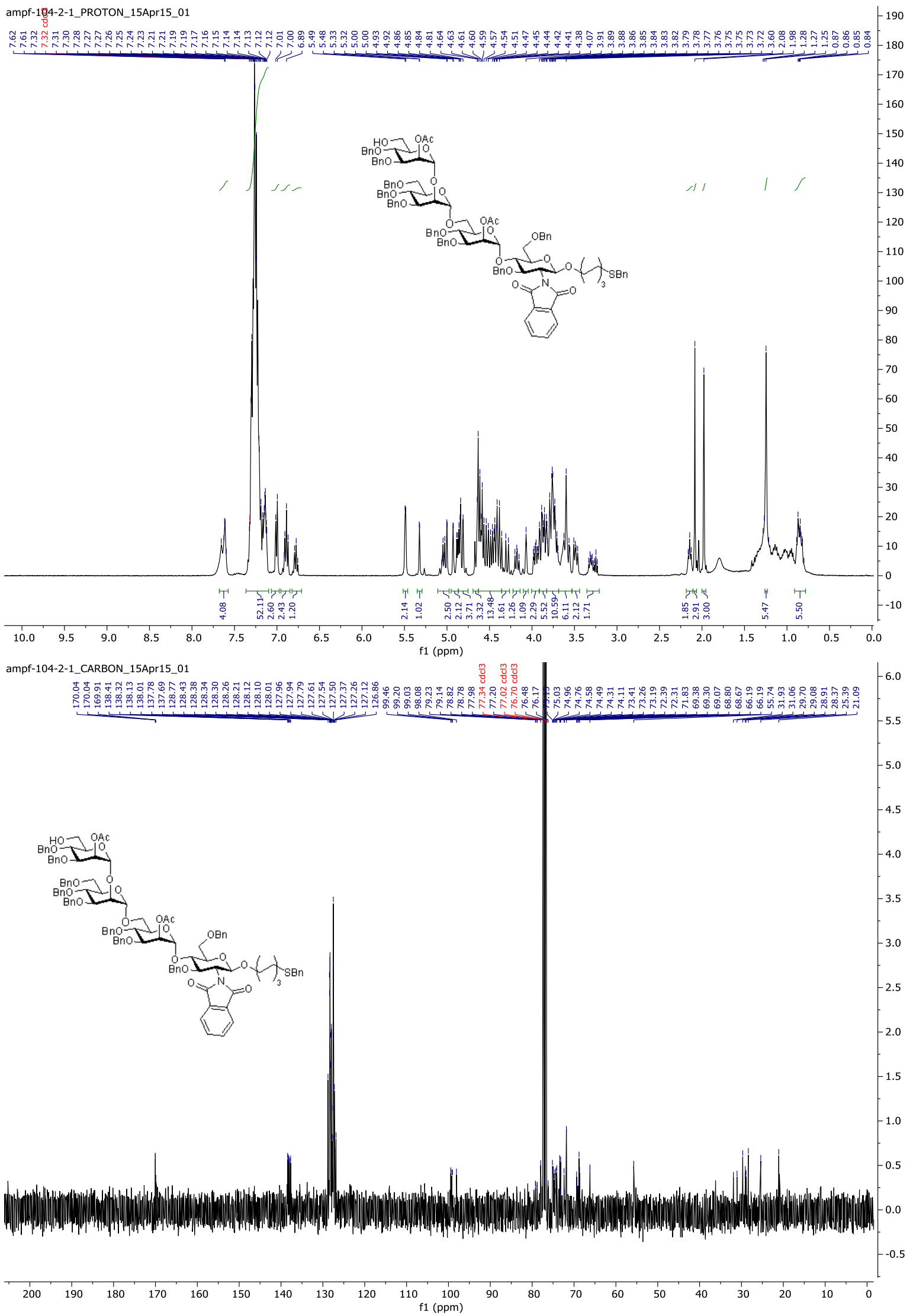


1-O-(6-thio)hexyl- $\alpha$-D-mannopyranosyl- $(1 \rightarrow 2)$ - $\alpha$-D-mannopyranosyl- $(1 \rightarrow 6)-\alpha-D$ mannopyranosyl-(1 $\rightarrow 4)$-2-amino-2-deoxy- $\beta$-D-glucopyranoside $(\mathbf{1})$

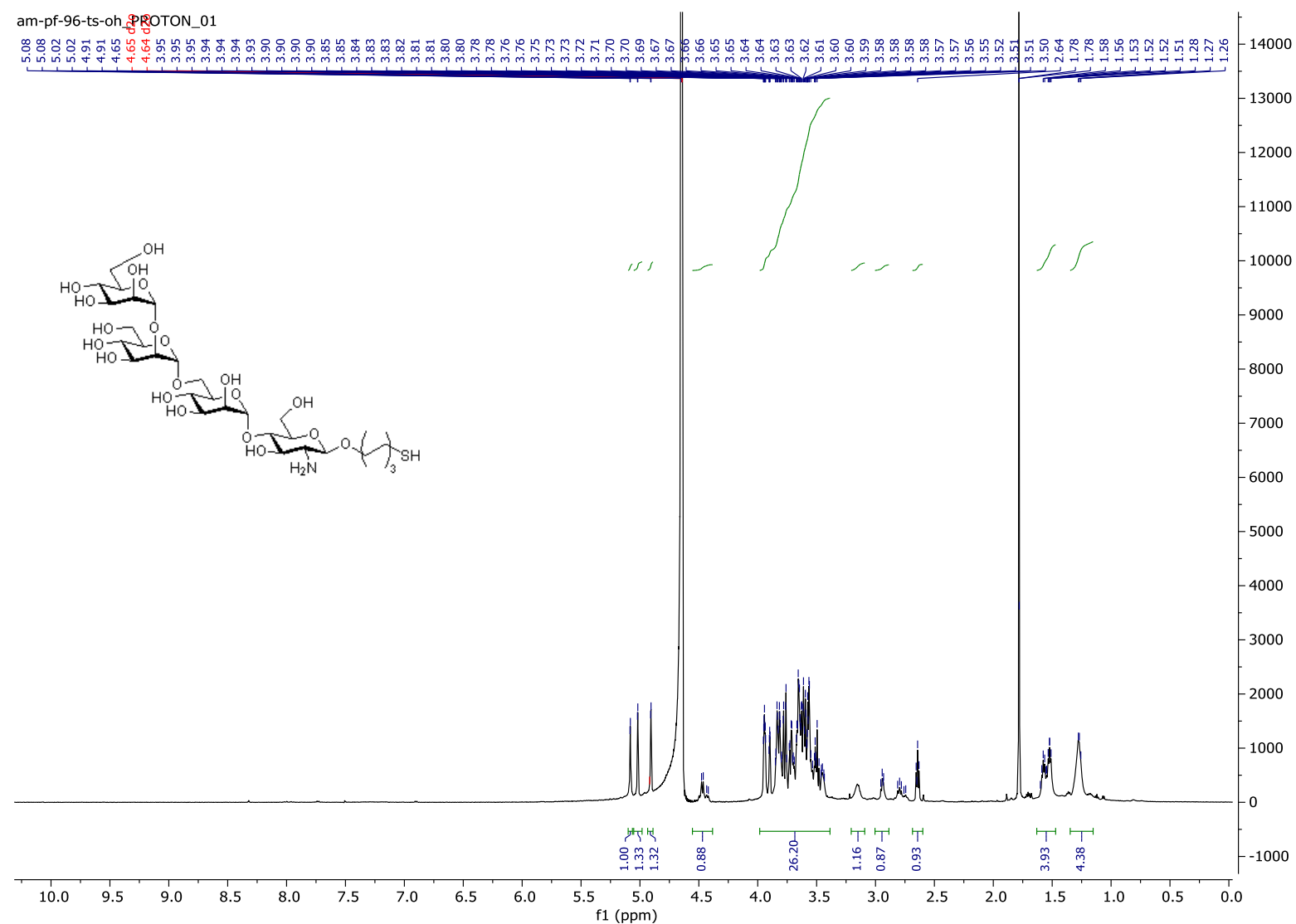

am-pf-96-ts-oh_CARBON_01
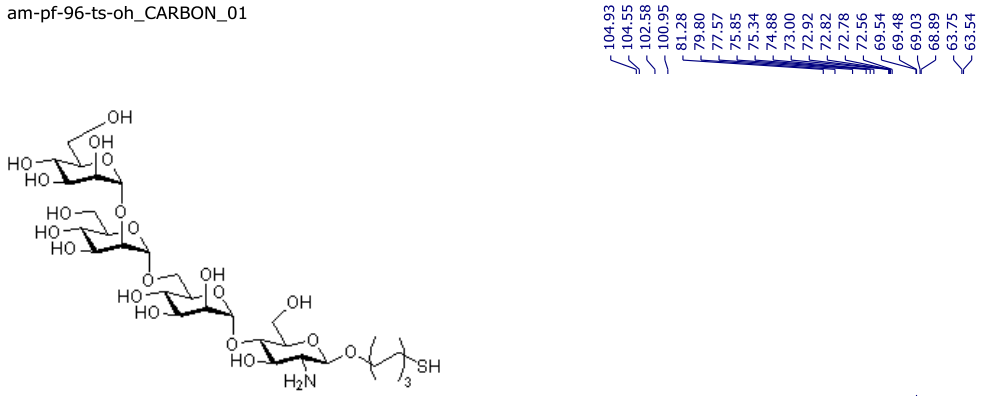

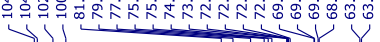

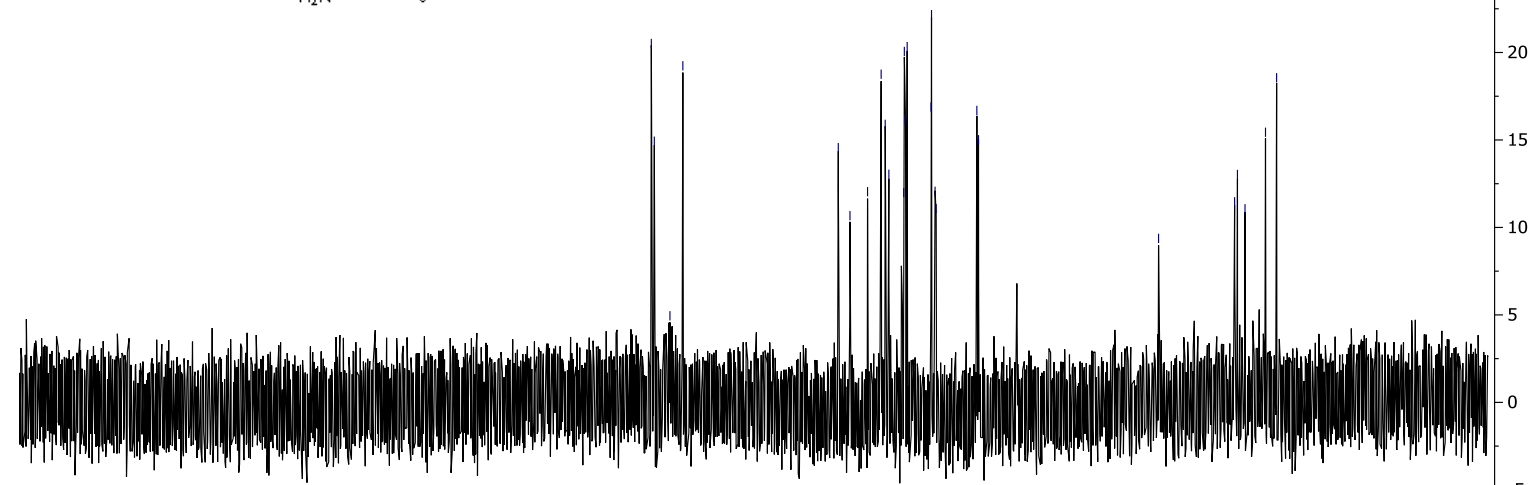




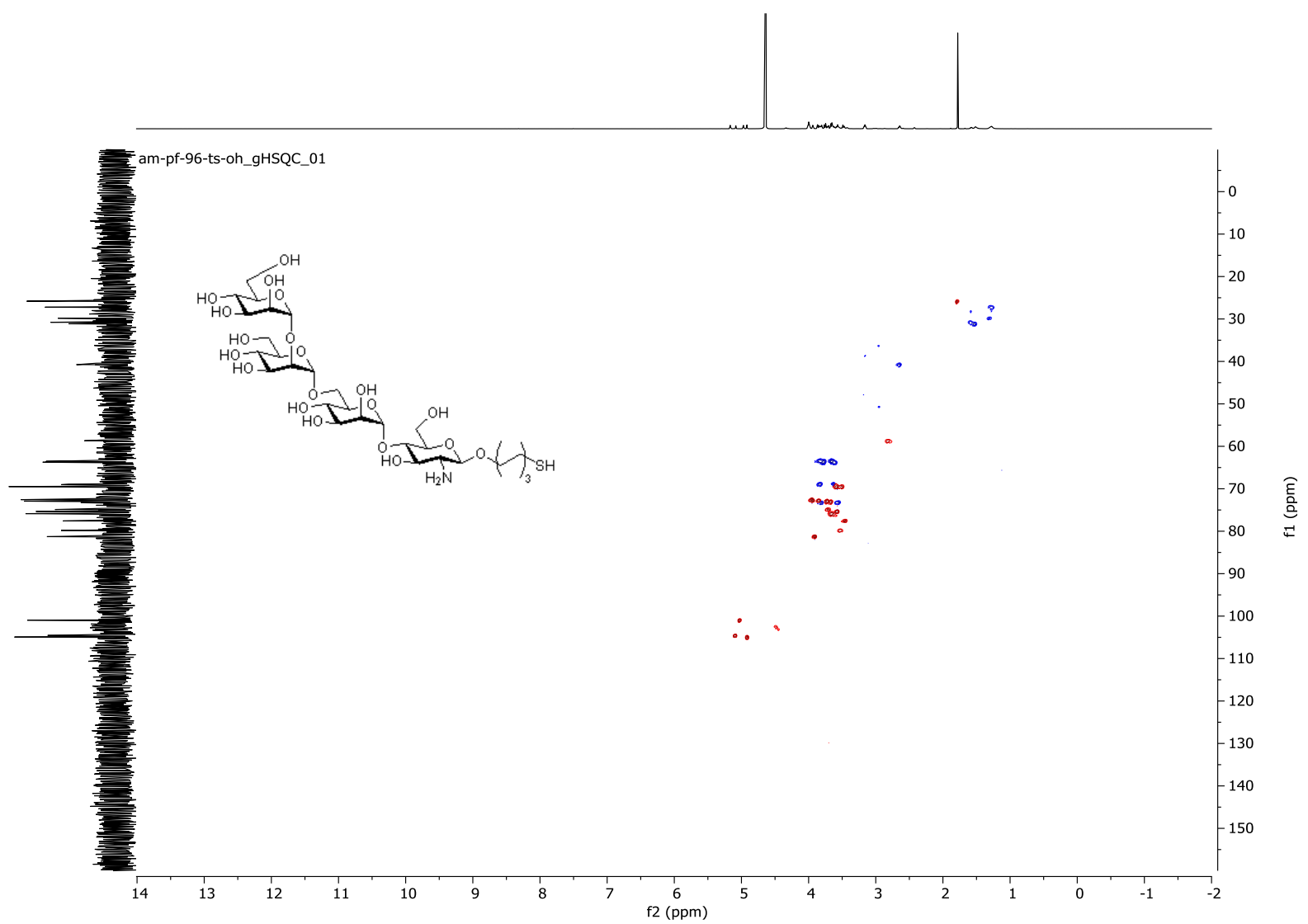

1-O-(6-thiobenzyl)hexyl-2-O-Acetyl-3,4-di-O-benzyl-6-O-(2-N-benzyloxycarbonyl) aminoethyl-phosphonato- $\alpha$-D-mannopyranosyl-( $1 \rightarrow 2)-3,4,6$-tri-O-benzyl- $\alpha-D$ -

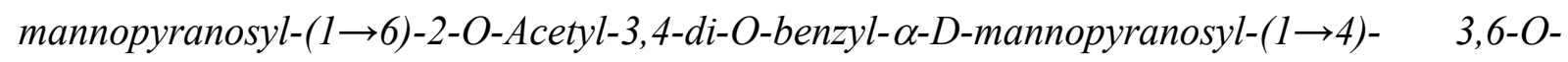
benzyl-2-deoxy-2-N-phthalimido- $\beta$-D-glucopyranoside (15)

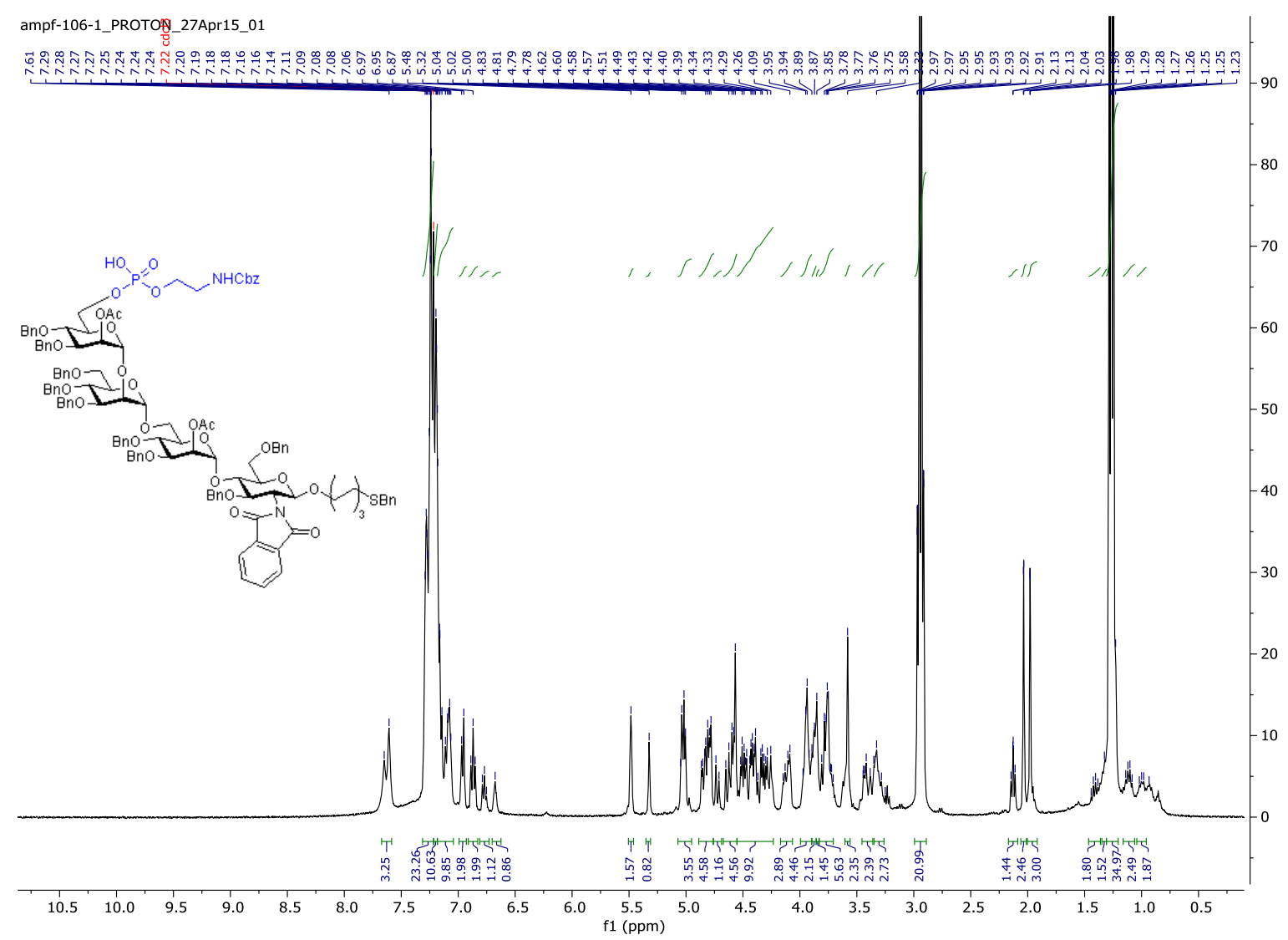




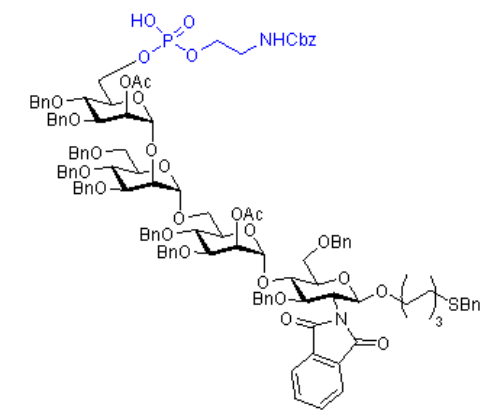

1-O-(6-thio)hexyl-6-O-aminoethyl-phosphonato- $\alpha$-D-mannopyranosyl- $(1 \rightarrow 2)-\alpha-D$ mannopyranosyl- $(1 \rightarrow 6)$ - $\alpha$-D-mannopyranosyl-( $1 \rightarrow 4)$-2-deoxy-2-amino- $\beta$ - $D$ glucopyranoside (2)

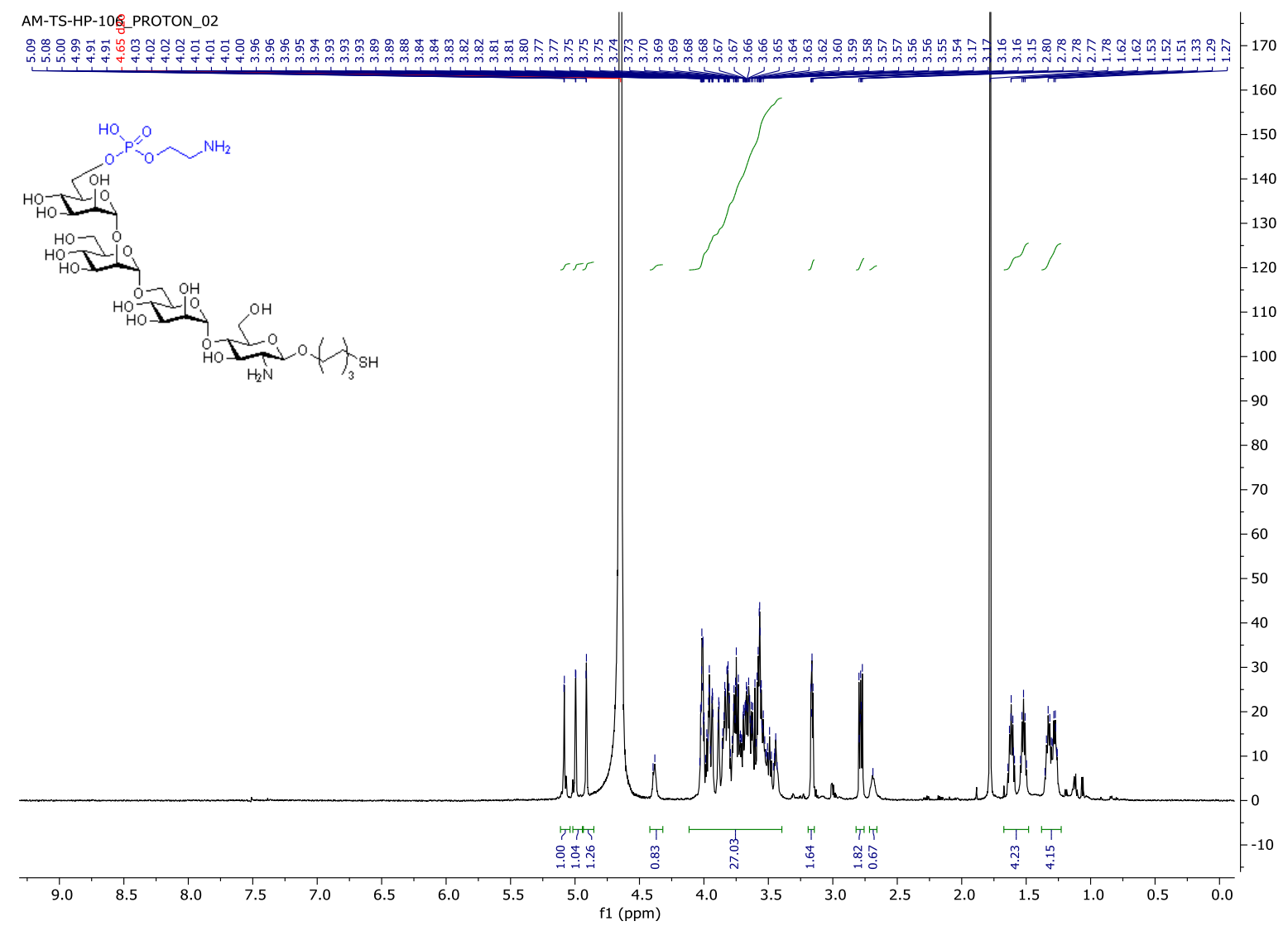



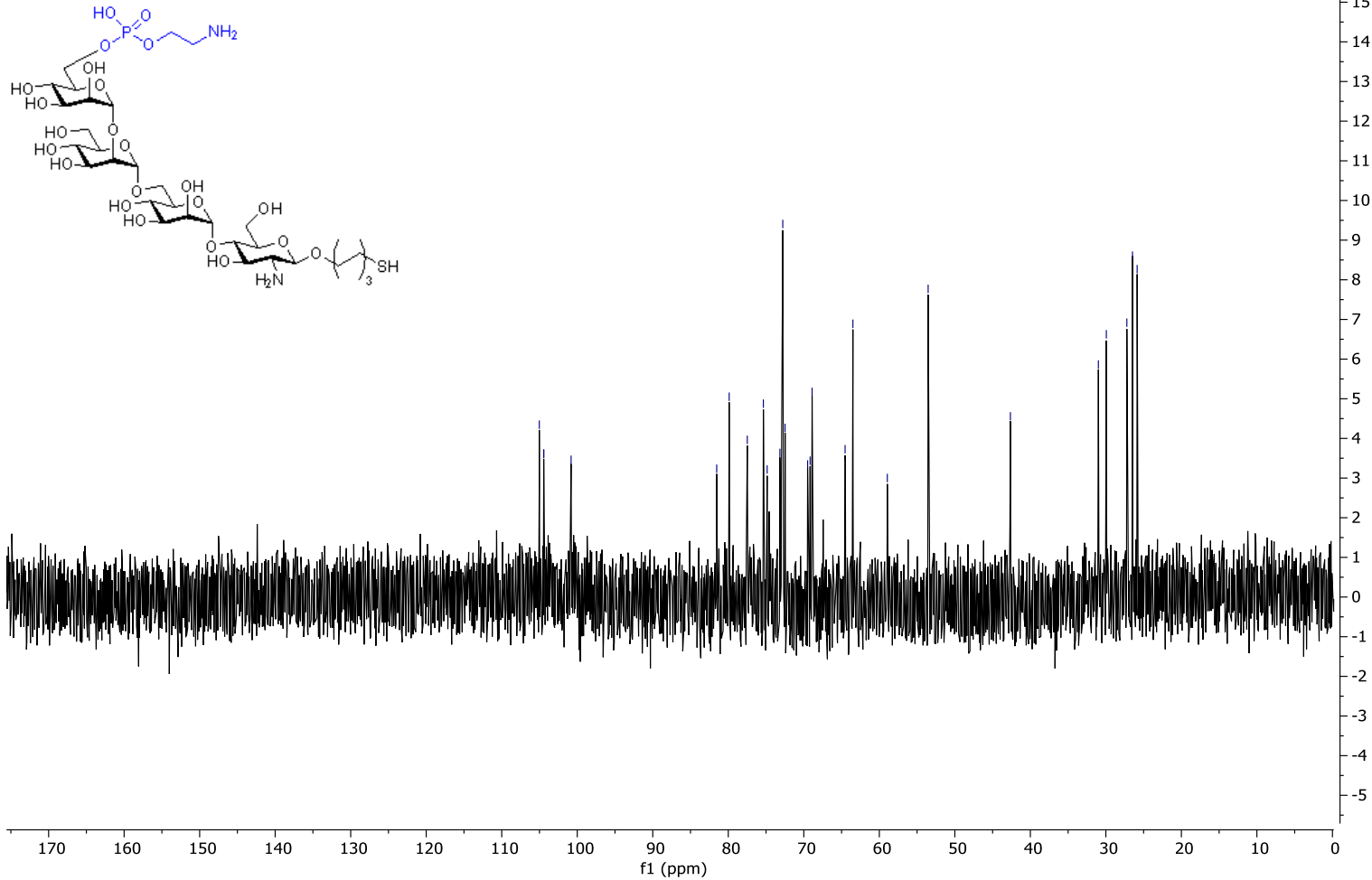

AM-TS-HP-106_PHOSPHORUS_01

\section{\begin{tabular}{l}
75 \\
70 \\
65 \\
60 \\
55 \\
50 \\
45 \\
40 \\
35 \\
30 \\
25 \\
20 \\
15 \\
10 \\
5 \\
\hline 0 \\
-5 \\
\hline
\end{tabular}}




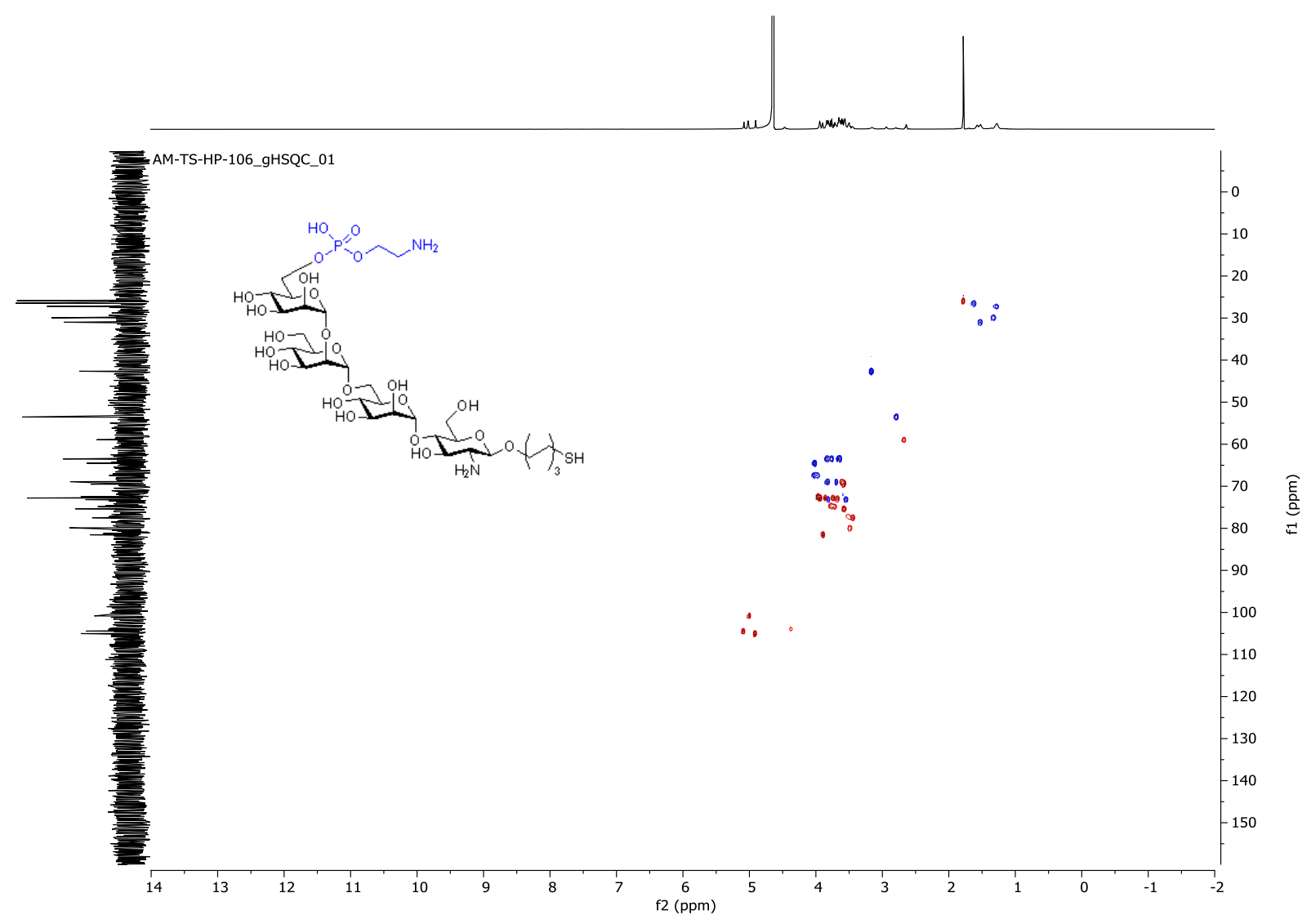

n-Allyl-3,4-di-O-benzyl-6-O-triisopropylsilyl- $\alpha$-D-mannopyranosyl-(1 $\rightarrow 2)-3,4,6-t r i-O-$ benzyl- $\alpha$-D-mannopyranoside (16)

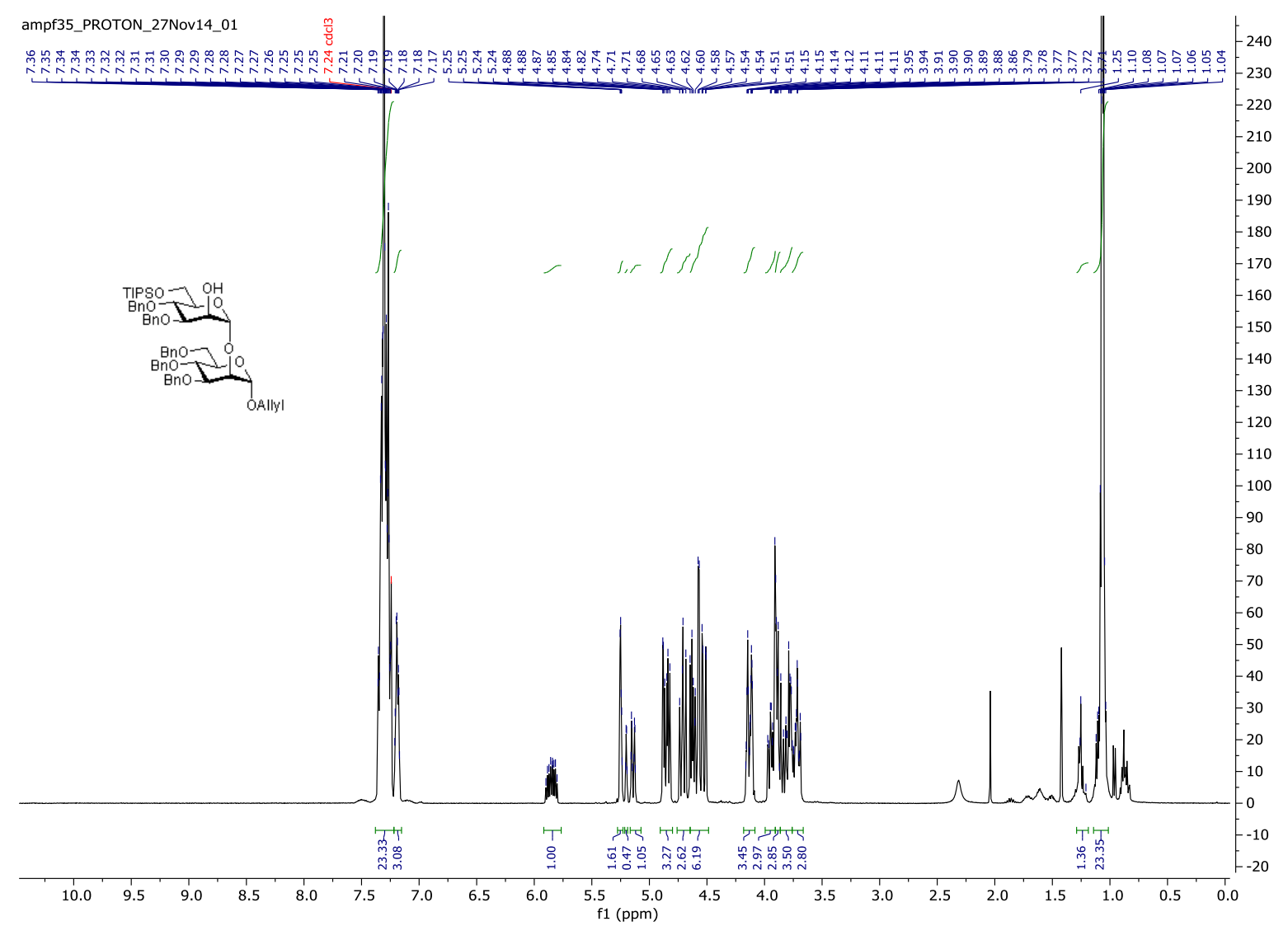




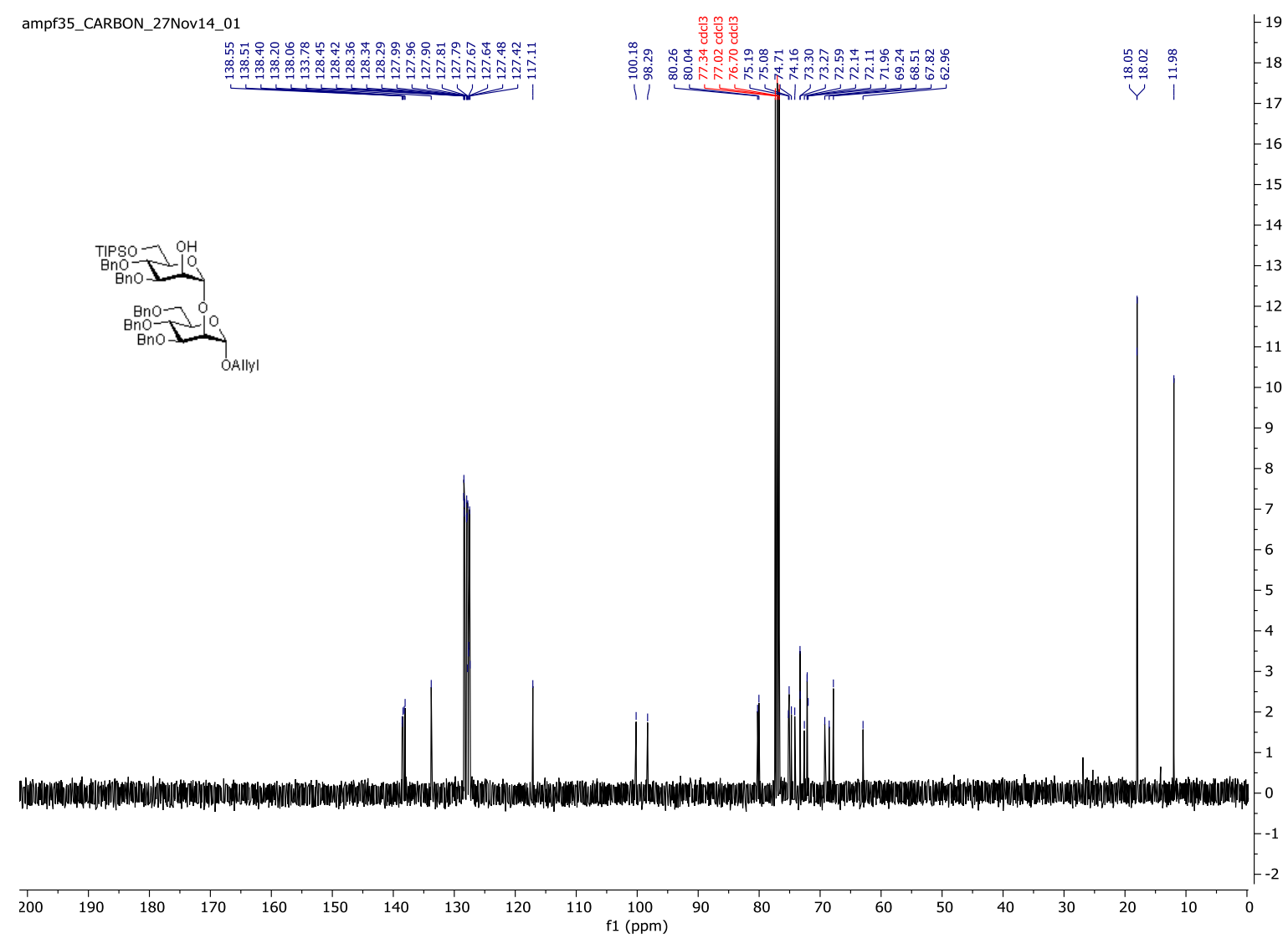

n-Allyl-2-O-acetyl-3,4,6-tri-O-benzyl- $\alpha$-D-mannopyranosyl- $(1 \rightarrow 2)-3,4-d i-O-b e n z y l-6-O$ triisopropylsilyl- $\alpha$-D-mannopyranosyl-(1-2)-3,4,6-tri-O-benzyl- $\alpha$-D-mannopyranoside (17)

ampf69_PROTON_27Jan15_-91

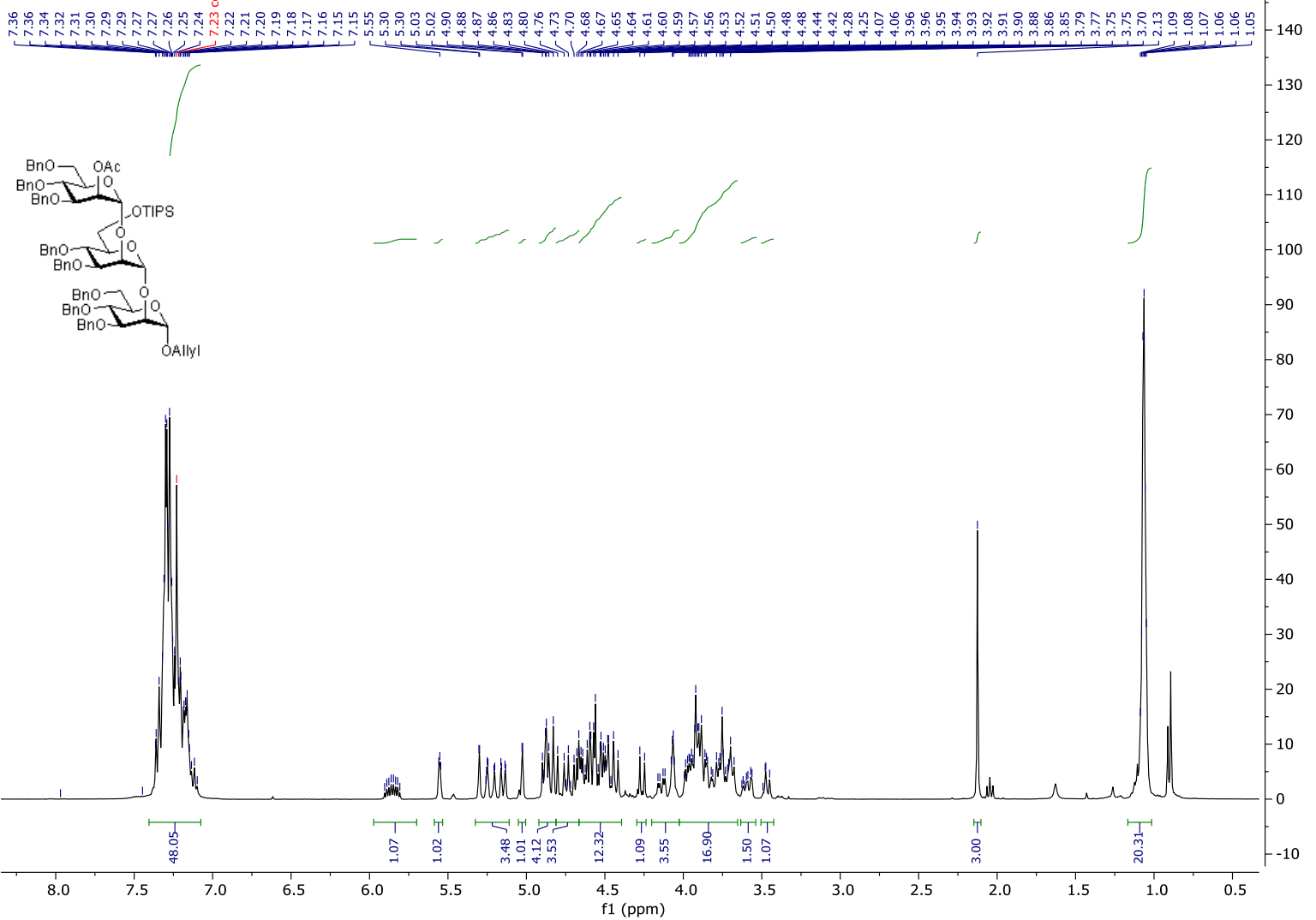




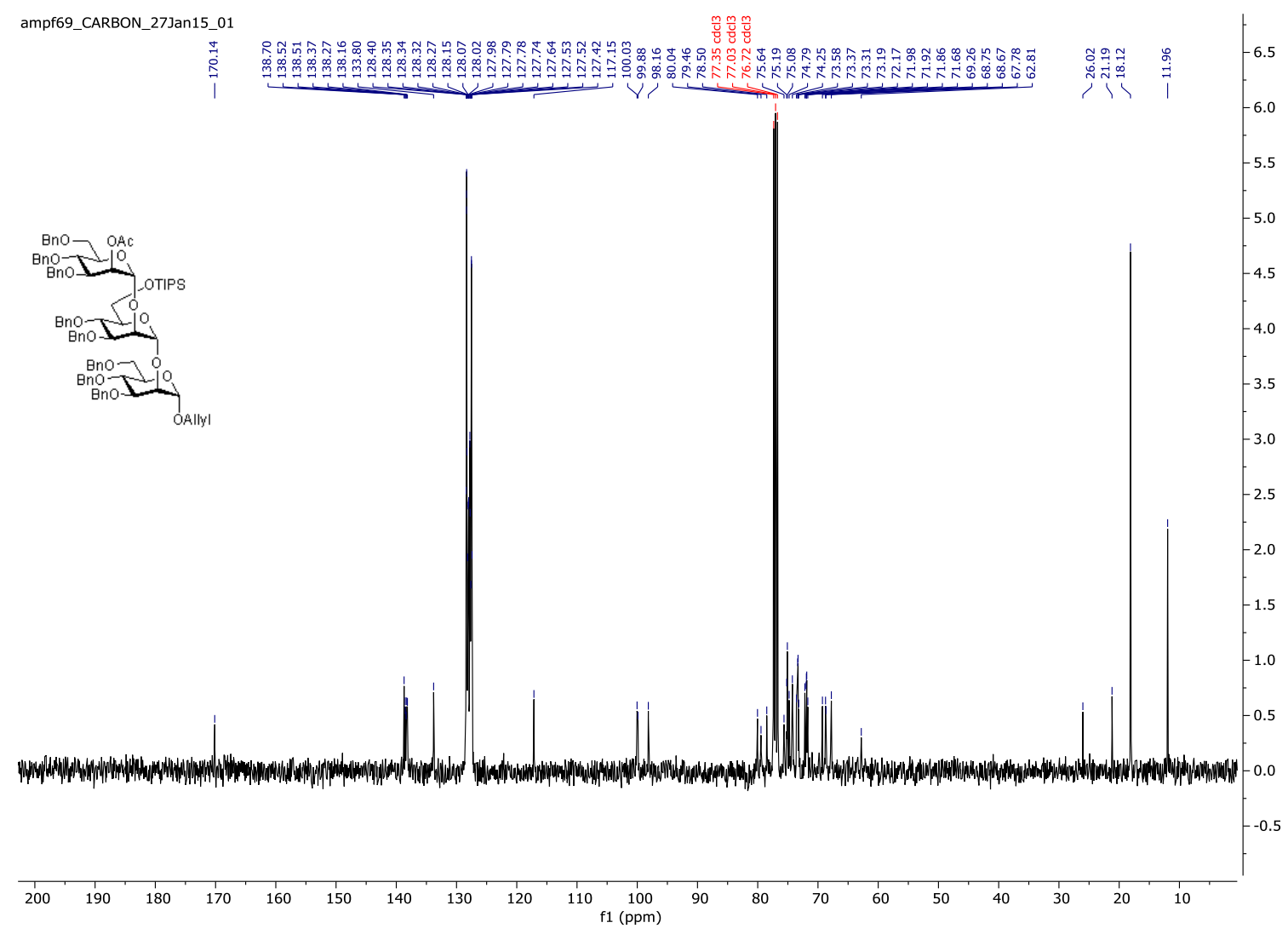

1-O-(6-thiobenzyl)hexyl-2-O-Acetyl-3,4,6-tri-O-benzyl- $\alpha$-D-mannopyranosyl-(1 $\rightarrow 2)-3,4-d i$ O-benzyl-6-O-triisopropylsilyl- $\alpha$-D-mannopyranosyl-(1 $\rightarrow 2)$-3,4,6-tri-O-benzyl- $\alpha$ - $D$ mannopyranosyl-(1 $\rightarrow 6)$-2-O-Acetyl-3,4-di-O-benzyl- $\alpha$-D-mannopyranosyl-( $1 \rightarrow 4)-\quad 3,6-O$ benzyl-2-deoxy-2-N-phthalimido- $\beta$-D-glucopyranoside (19)

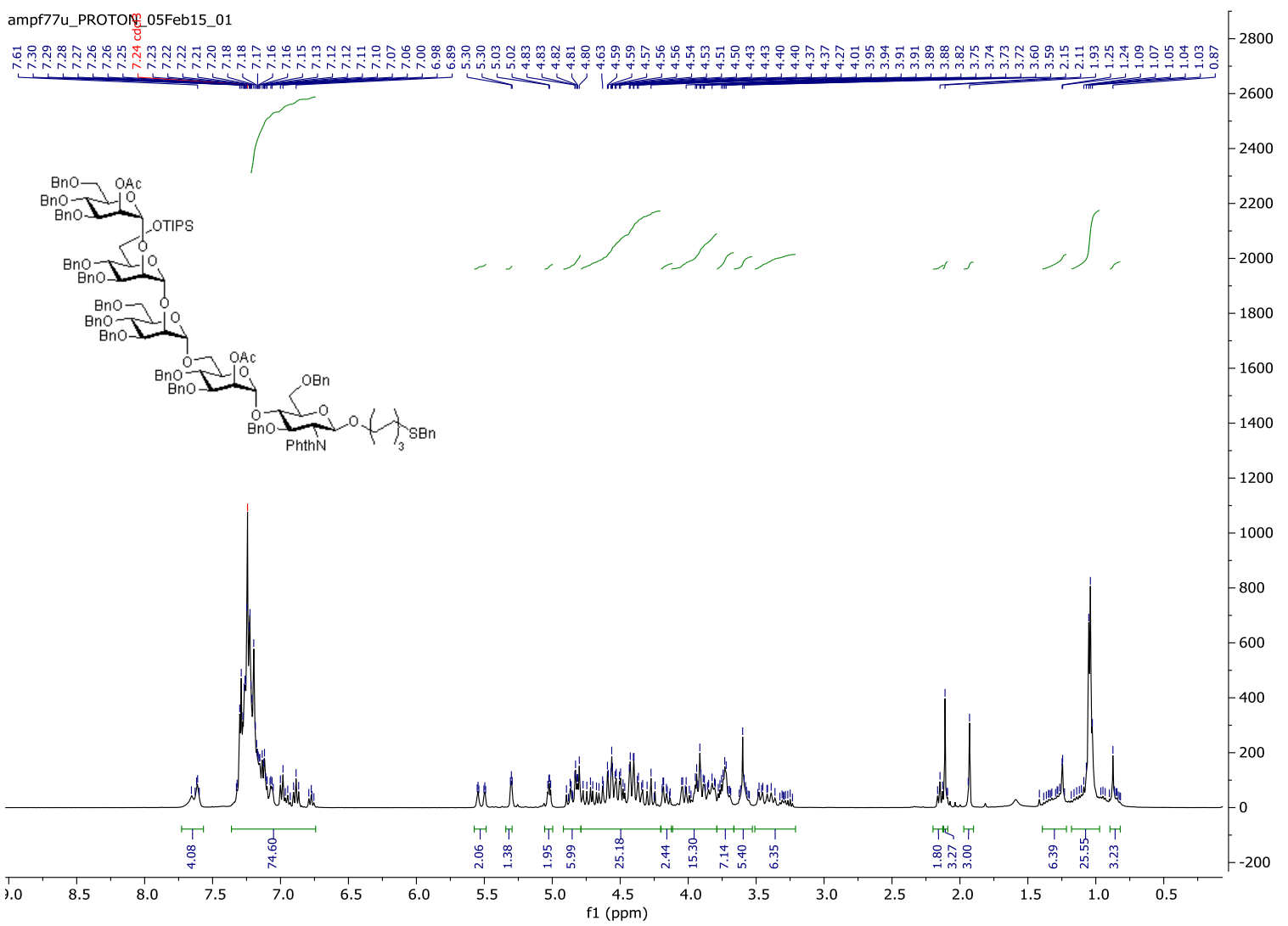




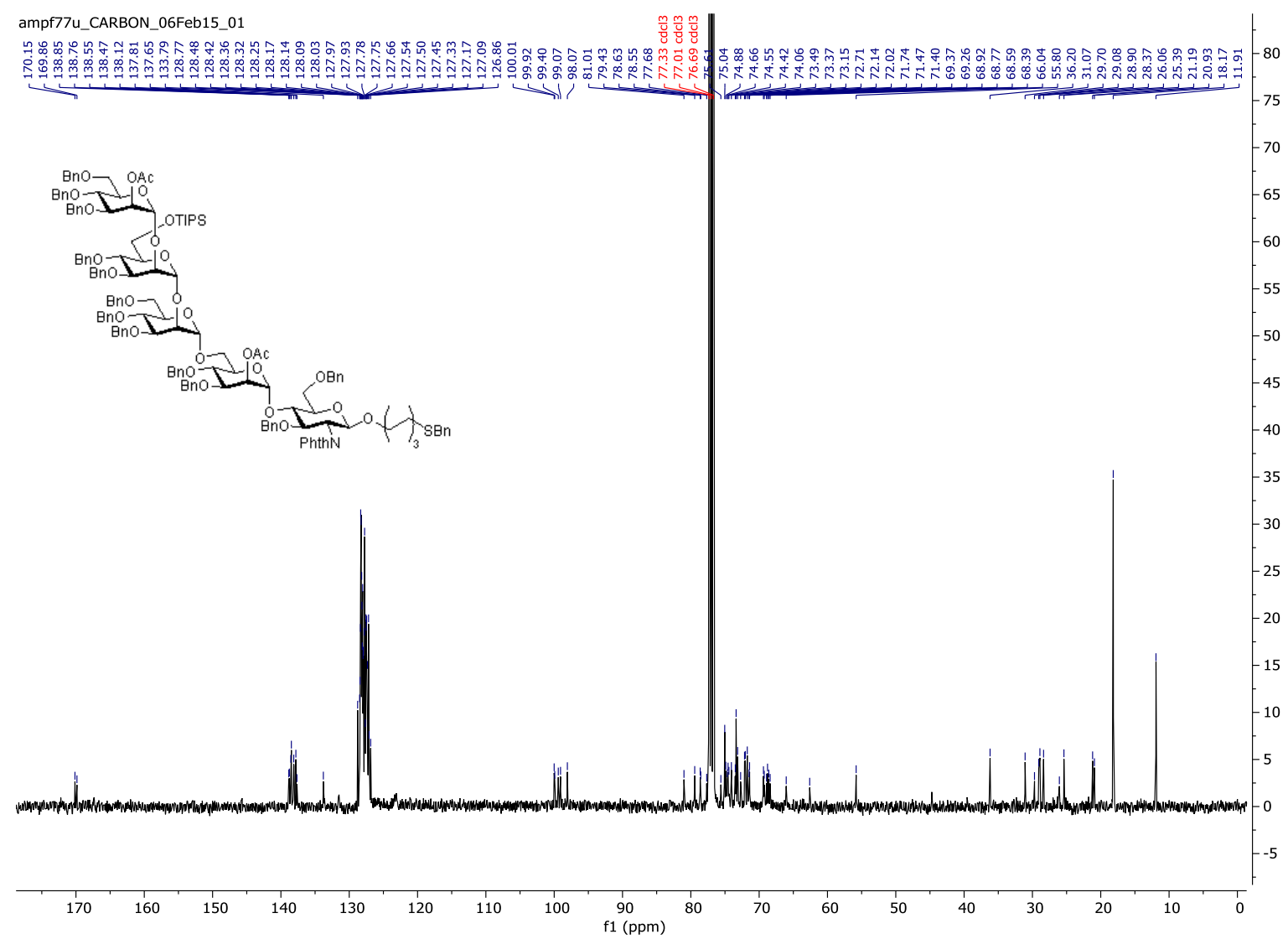

1-O-(6-thiobenzyl)hexyl-2-O-Acetyl-3,4,6-tri-O-benzyl- $\alpha$-D-mannopyranosyl-(1 $\rightarrow 2)-3,4-d i$ O-benzyl- $\alpha$-D-mannopyranosyl-( $1 \rightarrow 2)$-3,4,6-tri-O-benzyl- $\alpha$-D-mannopyranosyl- $(1 \rightarrow 6)-2-O$ Acetyl-3,4-di-O-benzyl- $\alpha$-D-mannopyranosyl-(1 $\rightarrow 4)$-3, 6-O-benzyl-2-deoxy-2-N-phthalimido$\beta$-D-glucopyranoside (20)

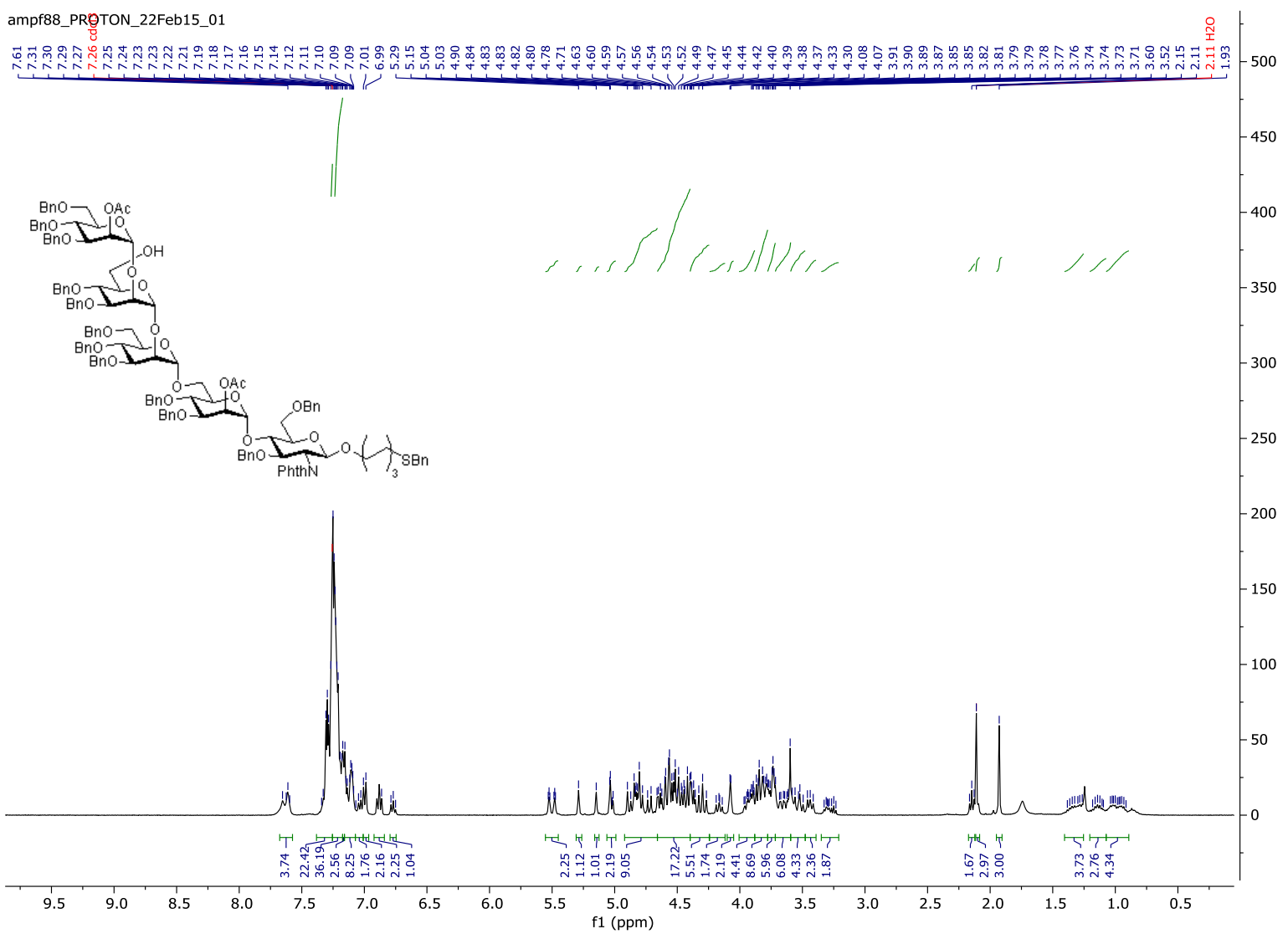




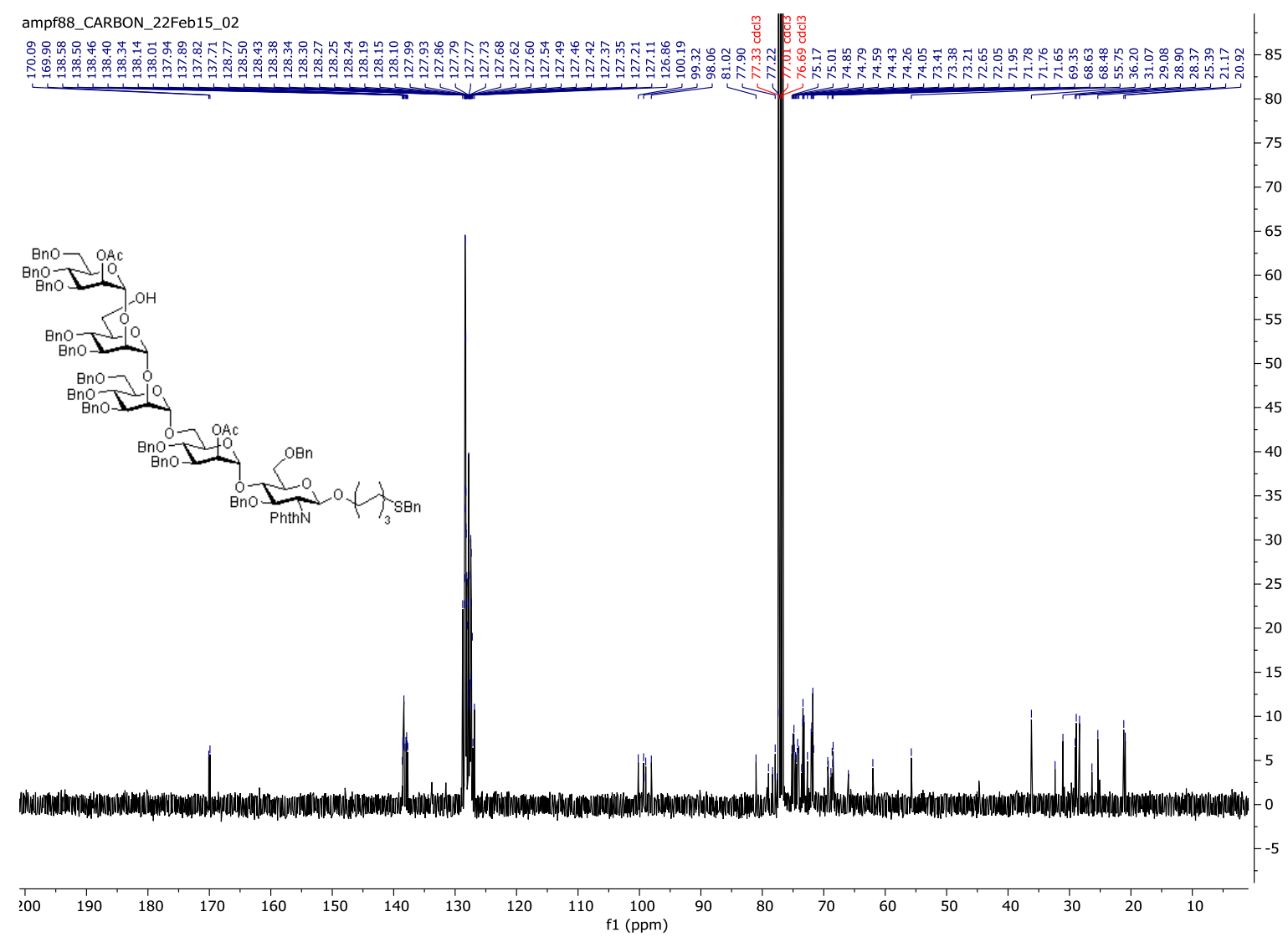

1-O-(6-thio)hexyl- $\alpha$-D-mannopyranosyl- $(1 \rightarrow 2)$ - $\alpha$-D-mannopyranosyl- $(1 \rightarrow 2)-\alpha-D$ -

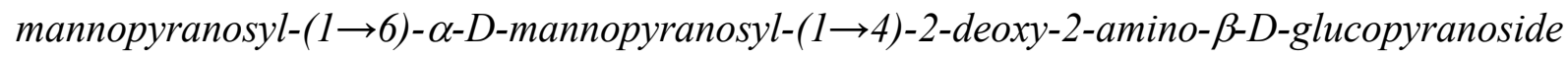
(3)

AM-PS-WOP-D2O

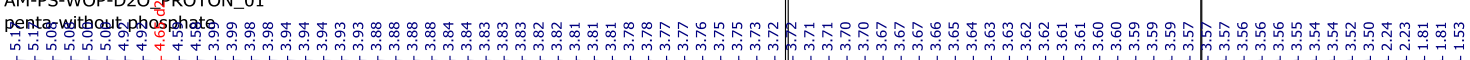

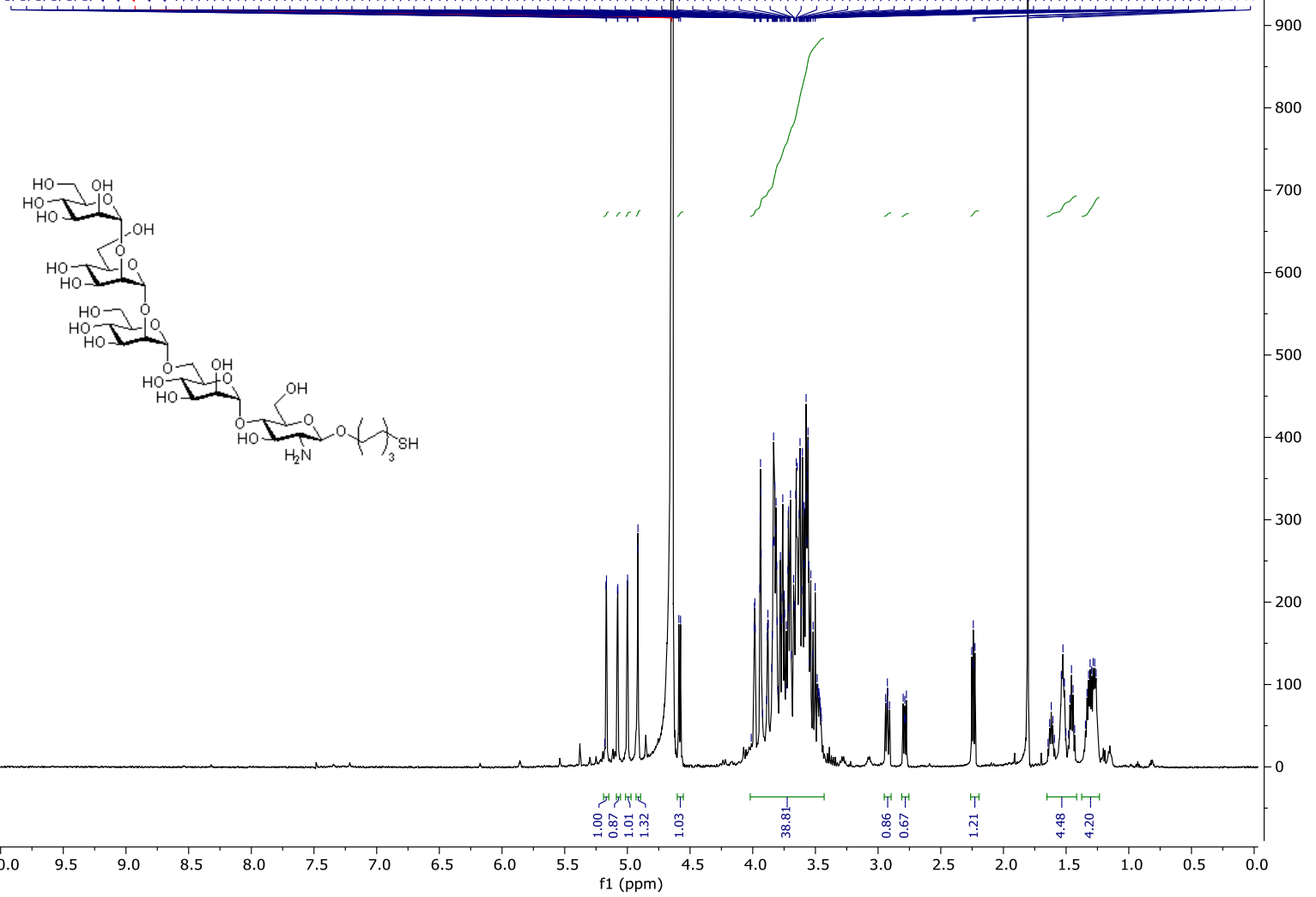



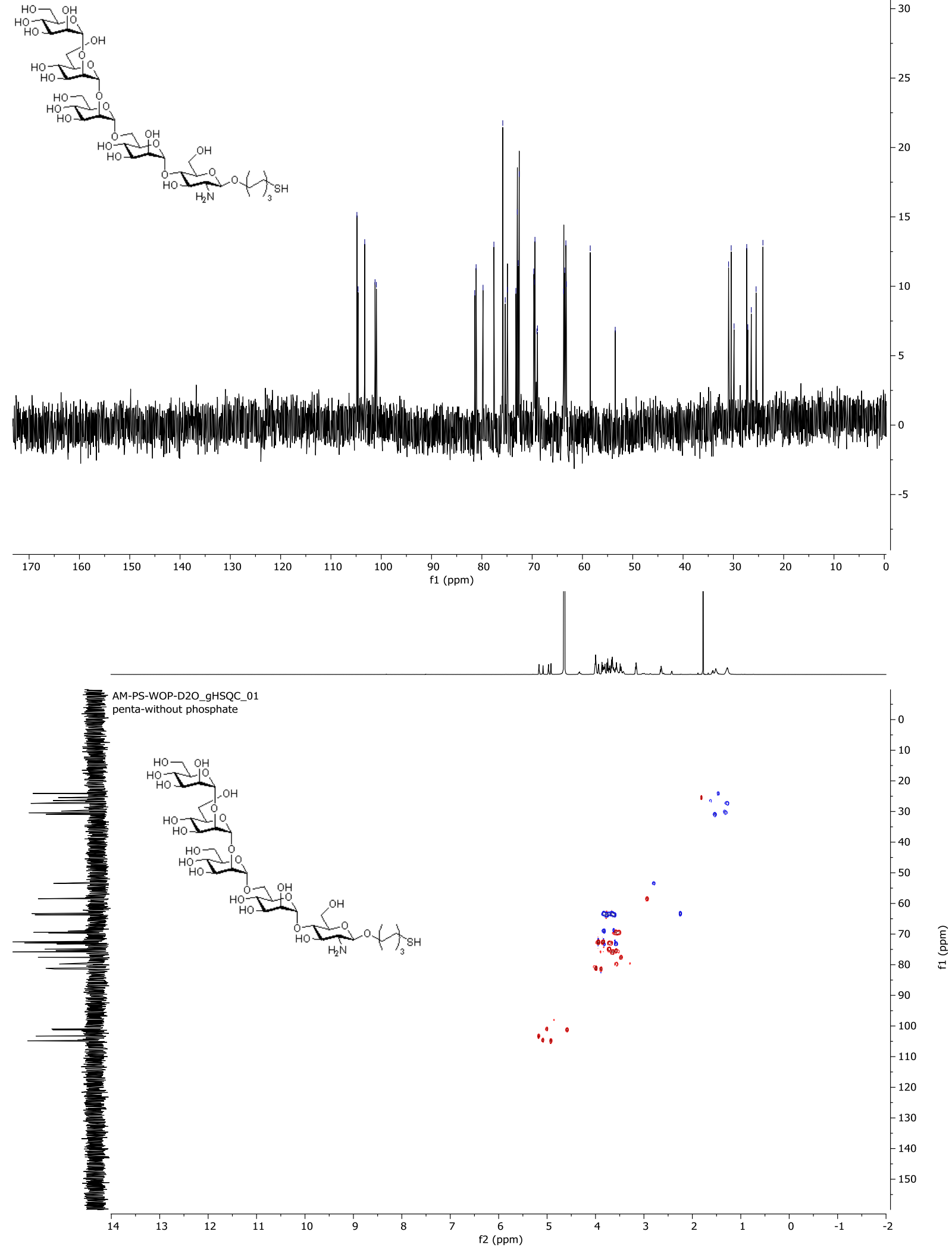
1-O-(6-thiobenzyl)hexyl-2-O-Acetyl-3,4,6-tri-O-benzyl- $\alpha$-D-mannopyranosyl-( $1 \rightarrow 2)$-3,4-diO-benzyl-6-O-(2-N-benzyloxycarbonyl) aminoethyl-phosphonato- $\alpha$-D-mannopyranosyl$(1 \rightarrow 2)$-3,4,6-tri-O-benzyl- $\alpha$-D-mannopyranosyl- $(1 \rightarrow 6)-2-O$-Acetyl-3,4-di-O-benzyl- $\alpha-D$ mannopyranosyl-(1 $\rightarrow 4$ )-3,6-O-benzyl-2-deoxy-2-N-phthalimido- $\beta$-D-glucopyranoside (21)

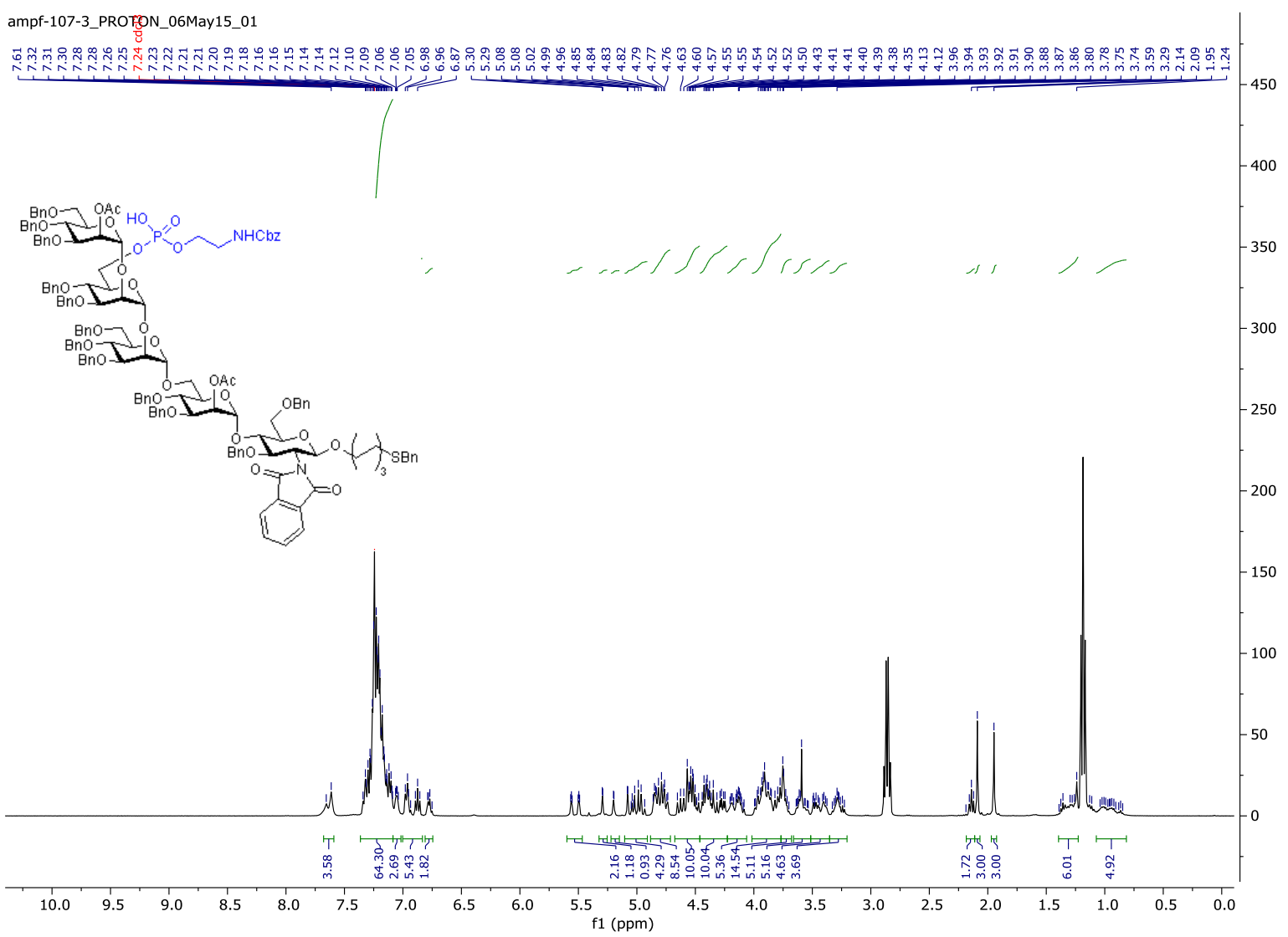



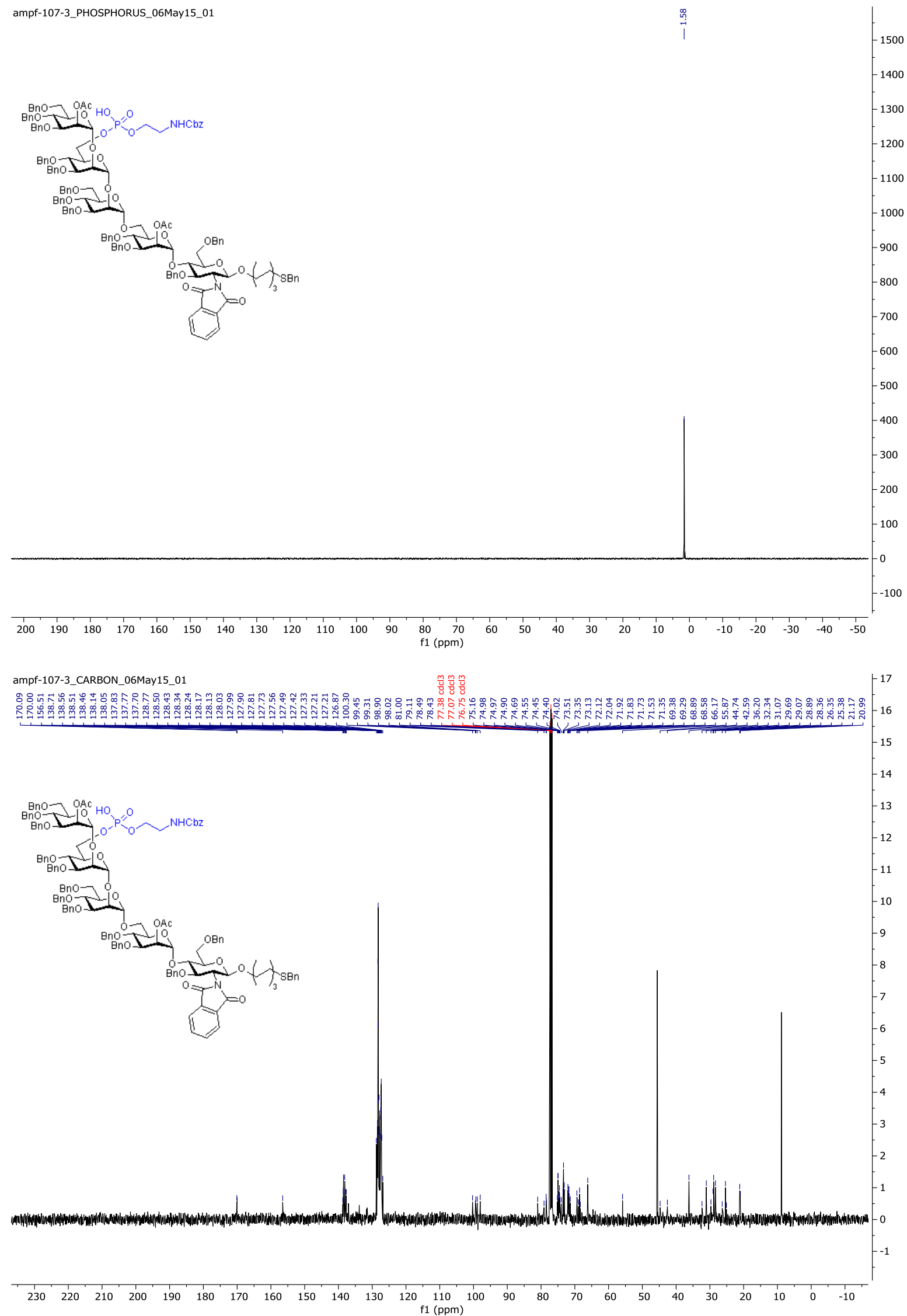
1-O-(6-thio)hexyl- $\alpha$-D-mannopyranosyl-(1 $\rightarrow 2)$-6-O-aminoethyl-phosphonato- $\alpha$ - $D$ -

mannopyranosyl-(1 $(\rightarrow 2)$ - $\alpha$-D-mannopyranosyl- $(1 \rightarrow 6)$ - $\alpha$-D-mannopyranosyl- $(1 \rightarrow 4)$-2-deoxy2-amino- $\beta$-D-glucopyranoside (4)

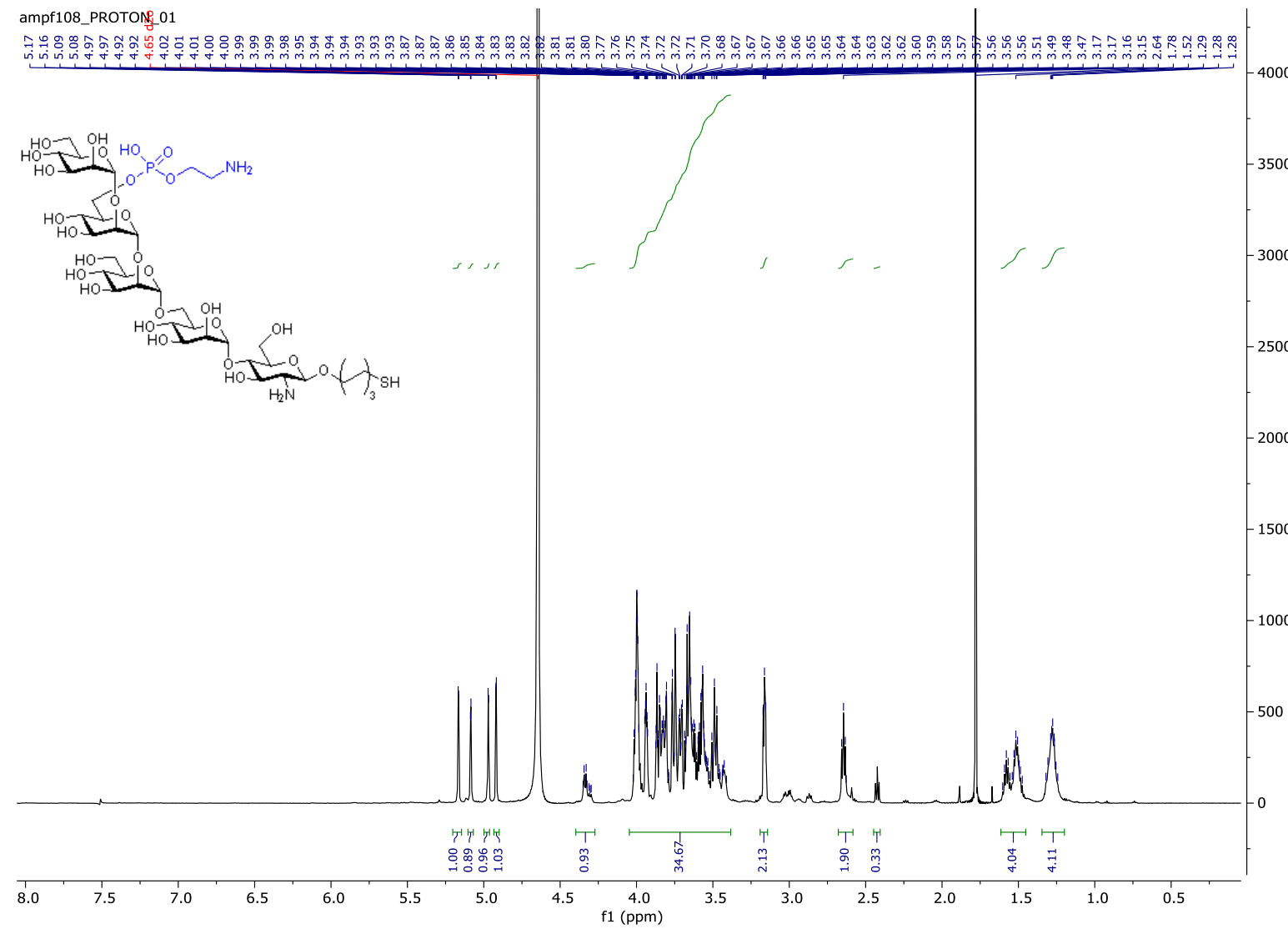

ampf108_PHOSPHORUS_01

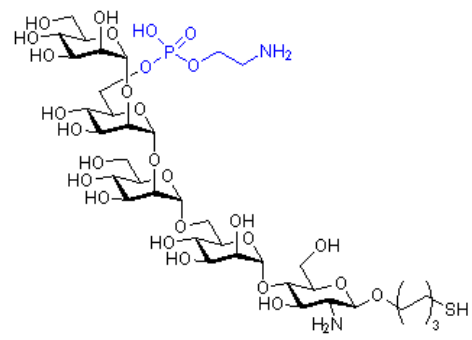



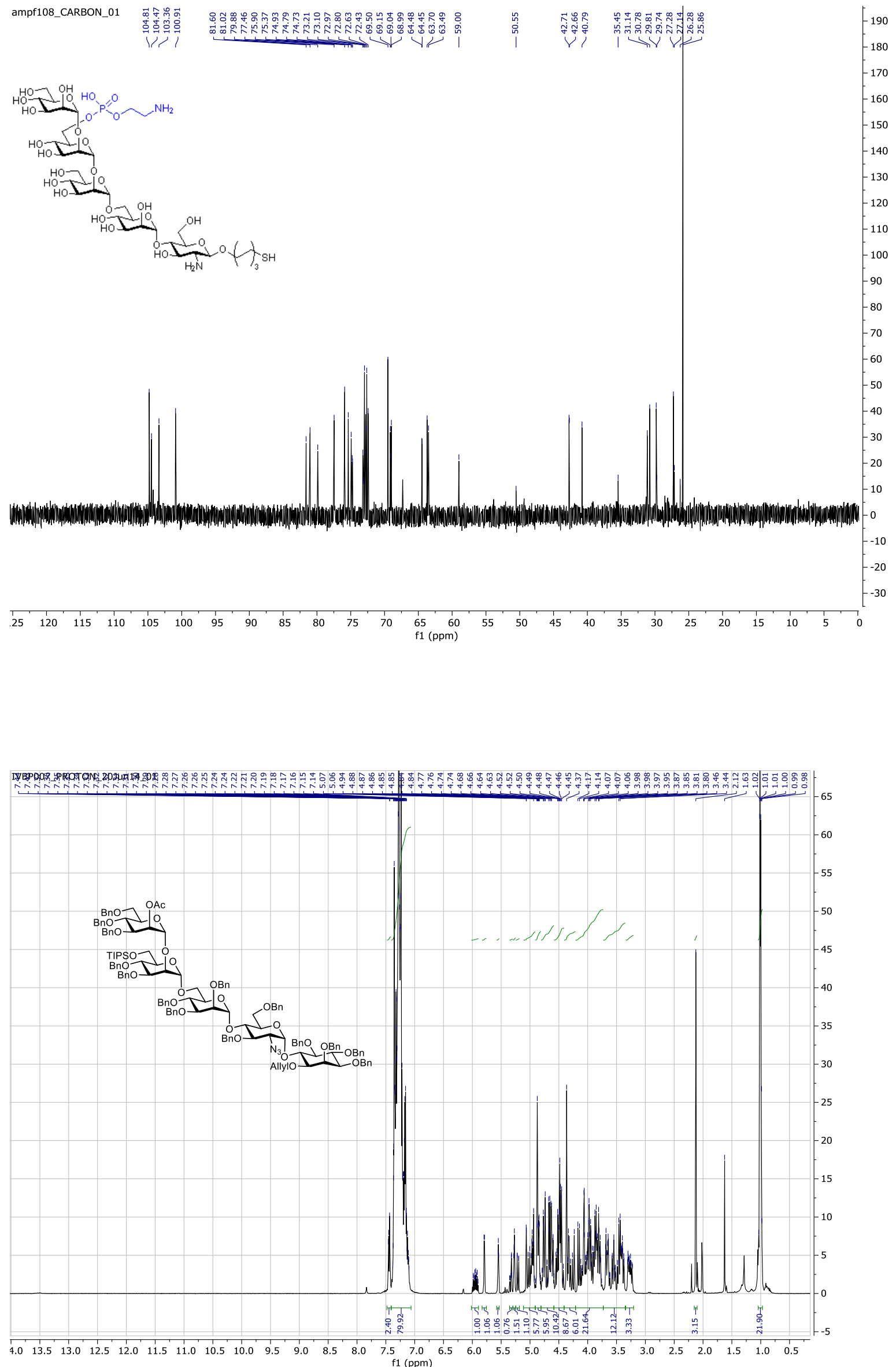

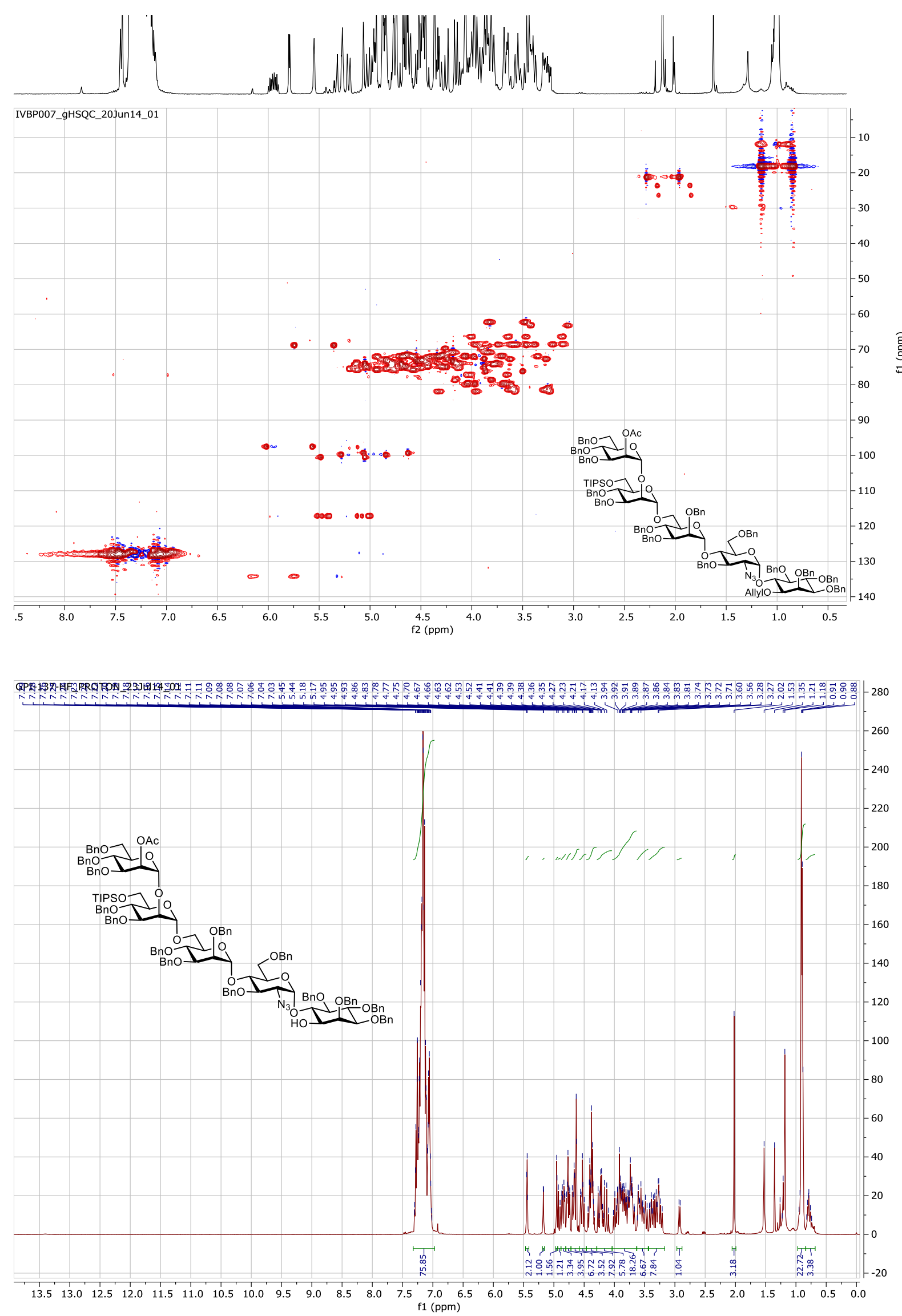

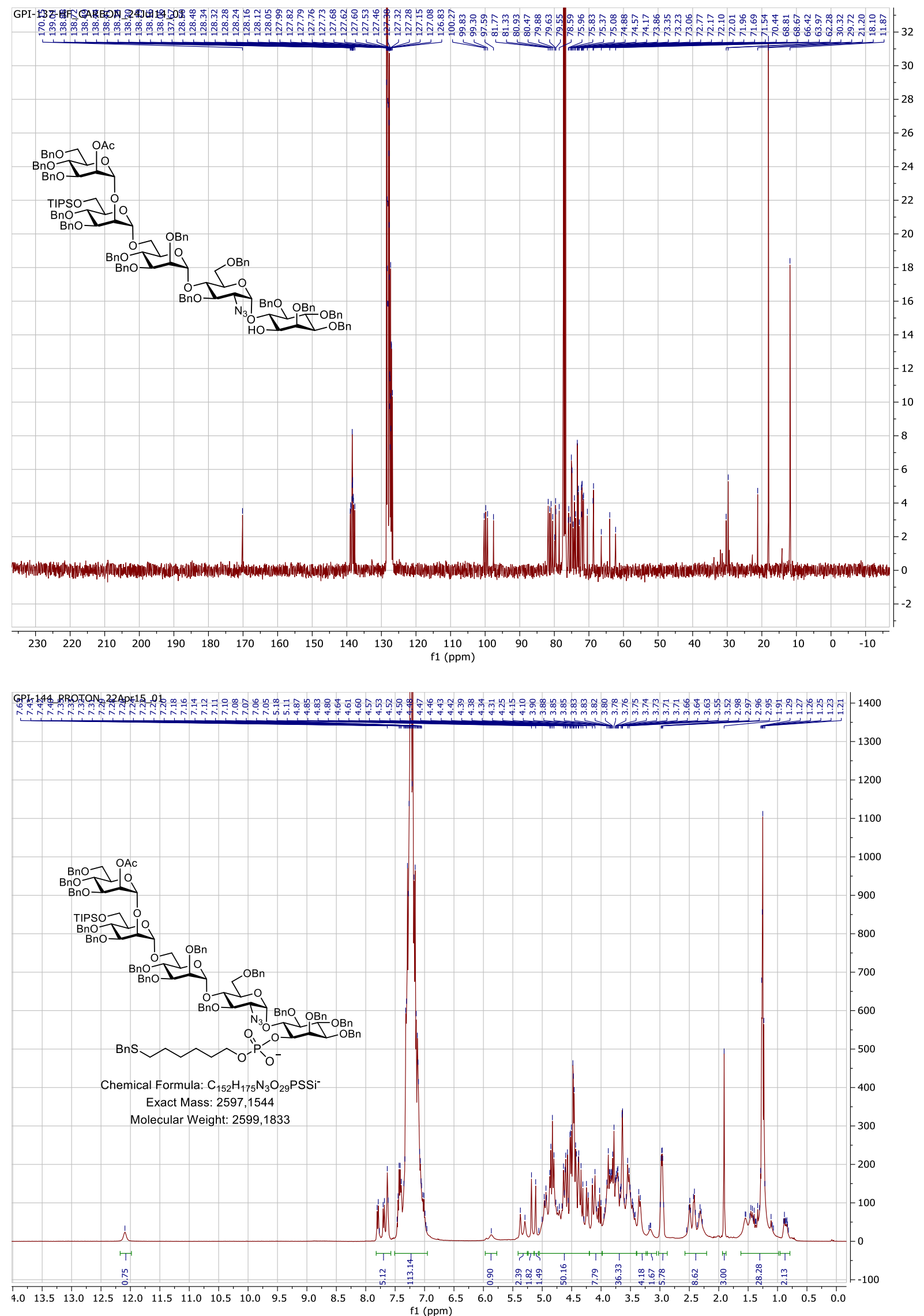


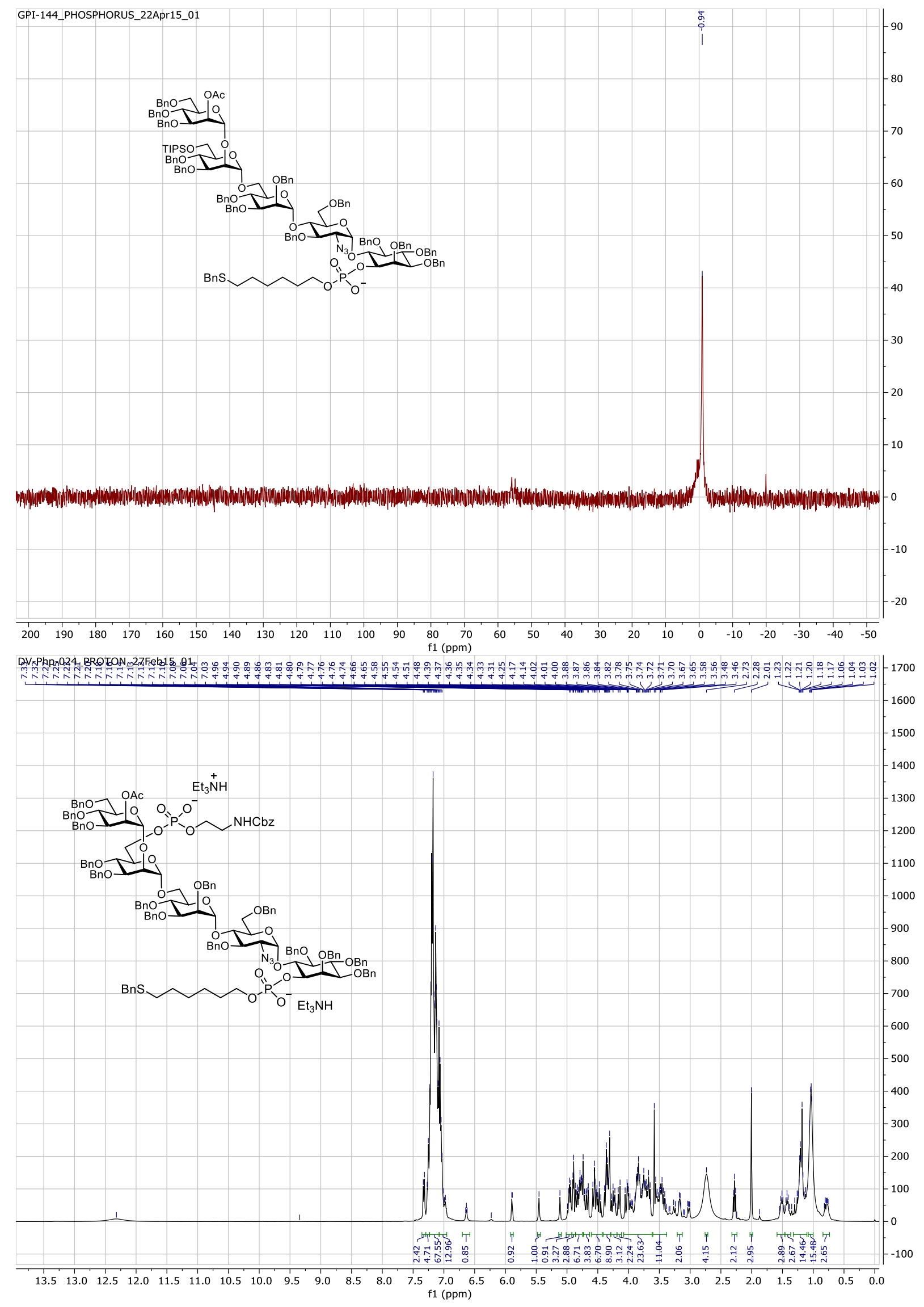

S54 


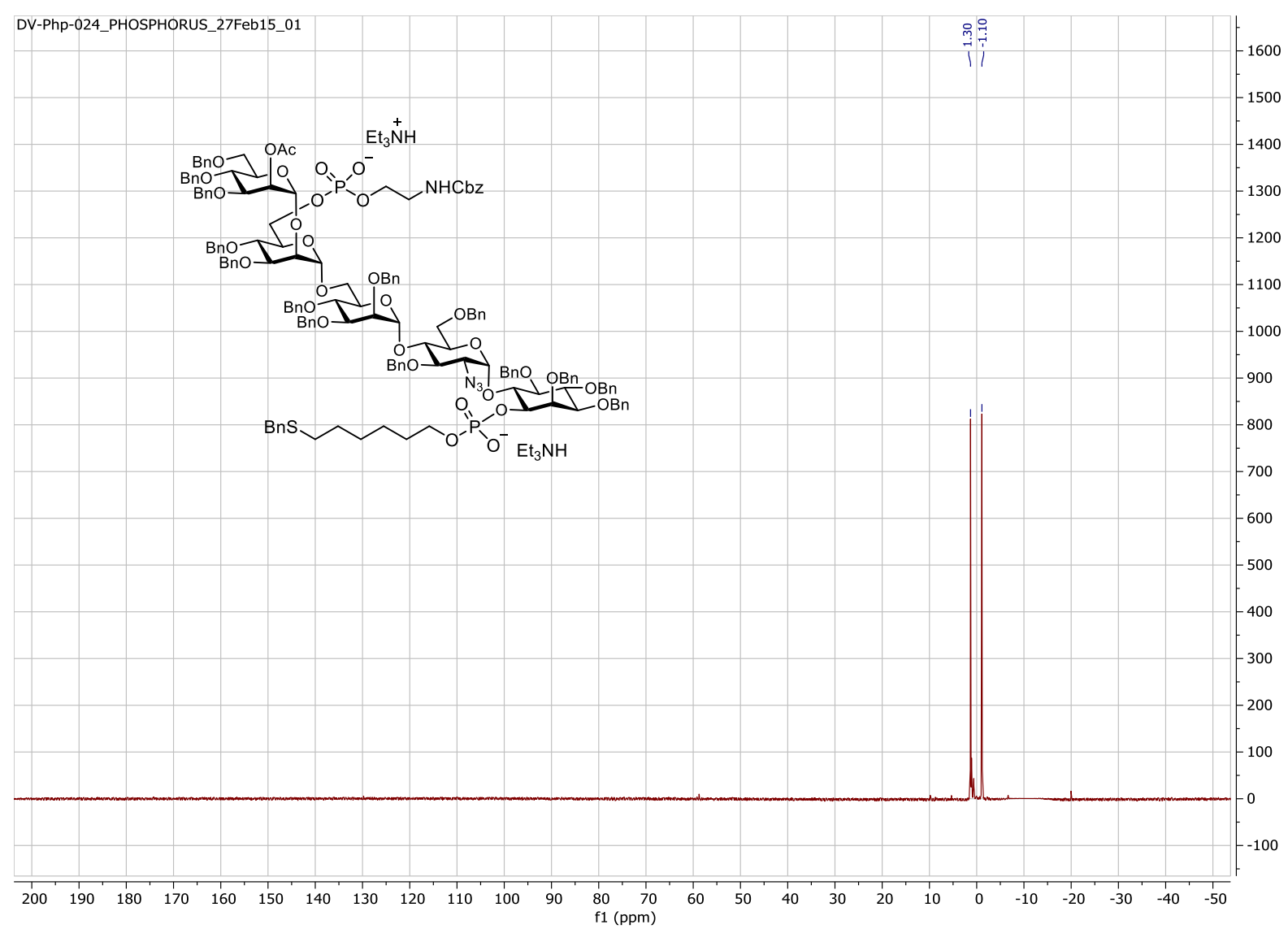



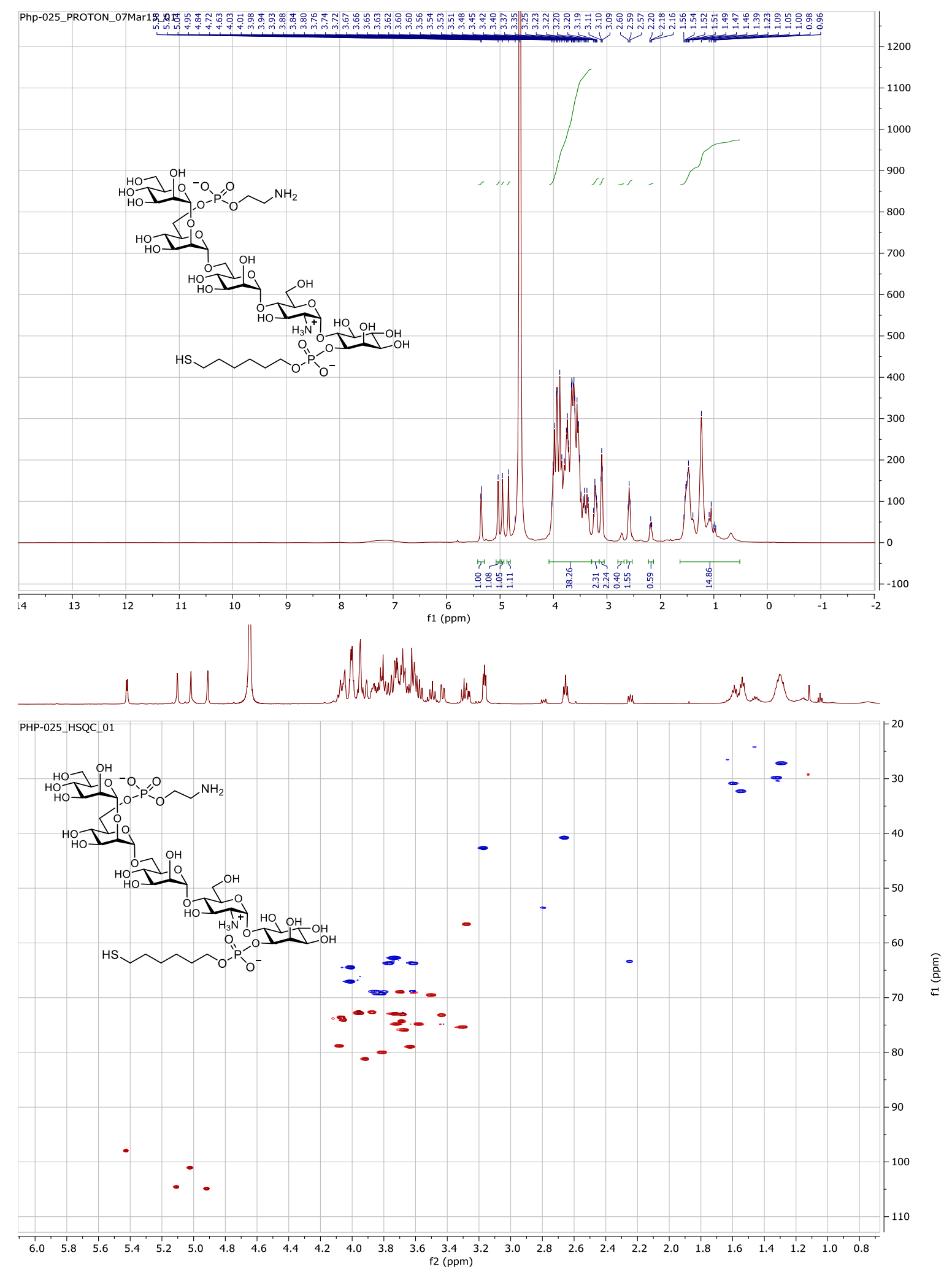

S56 

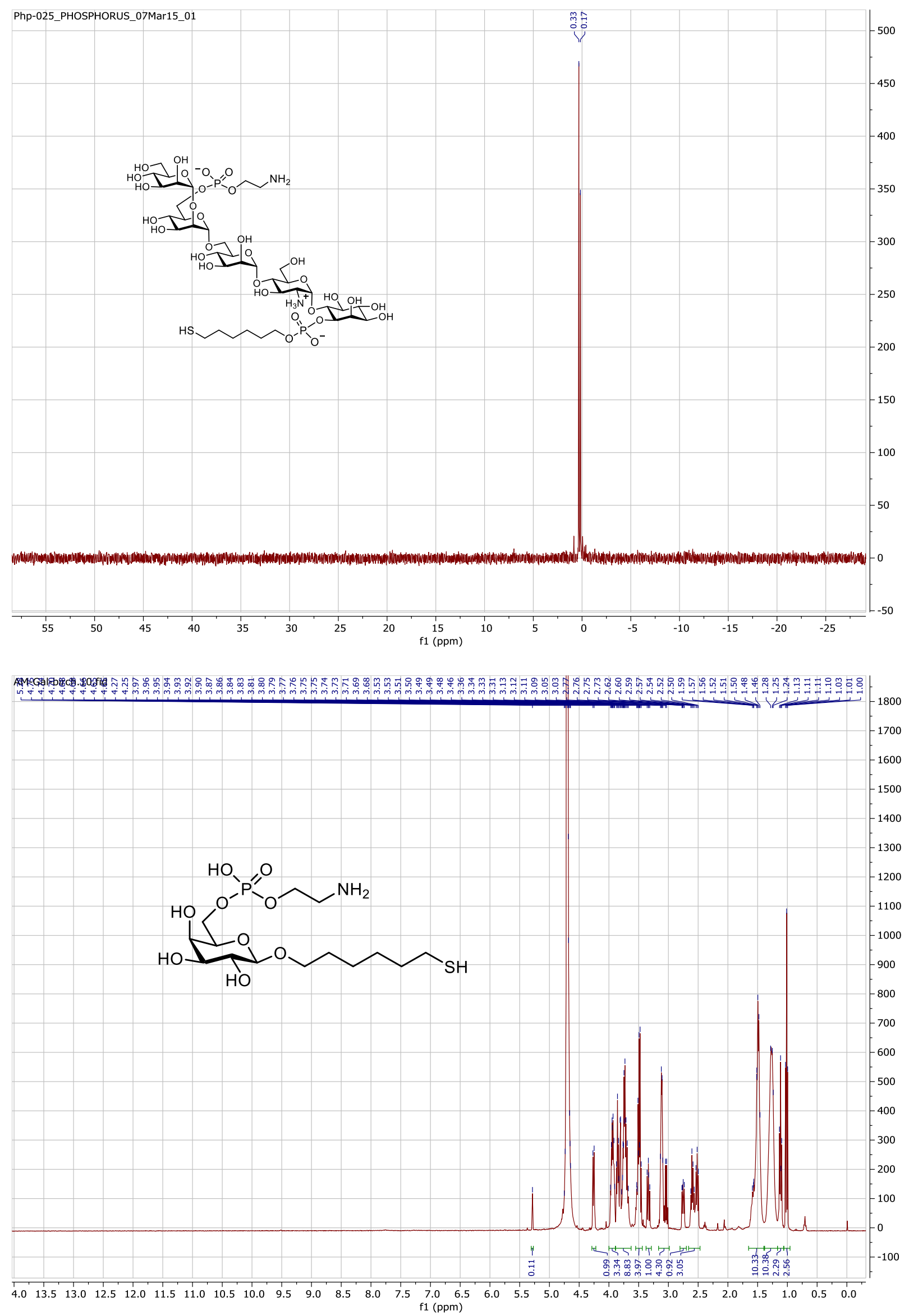


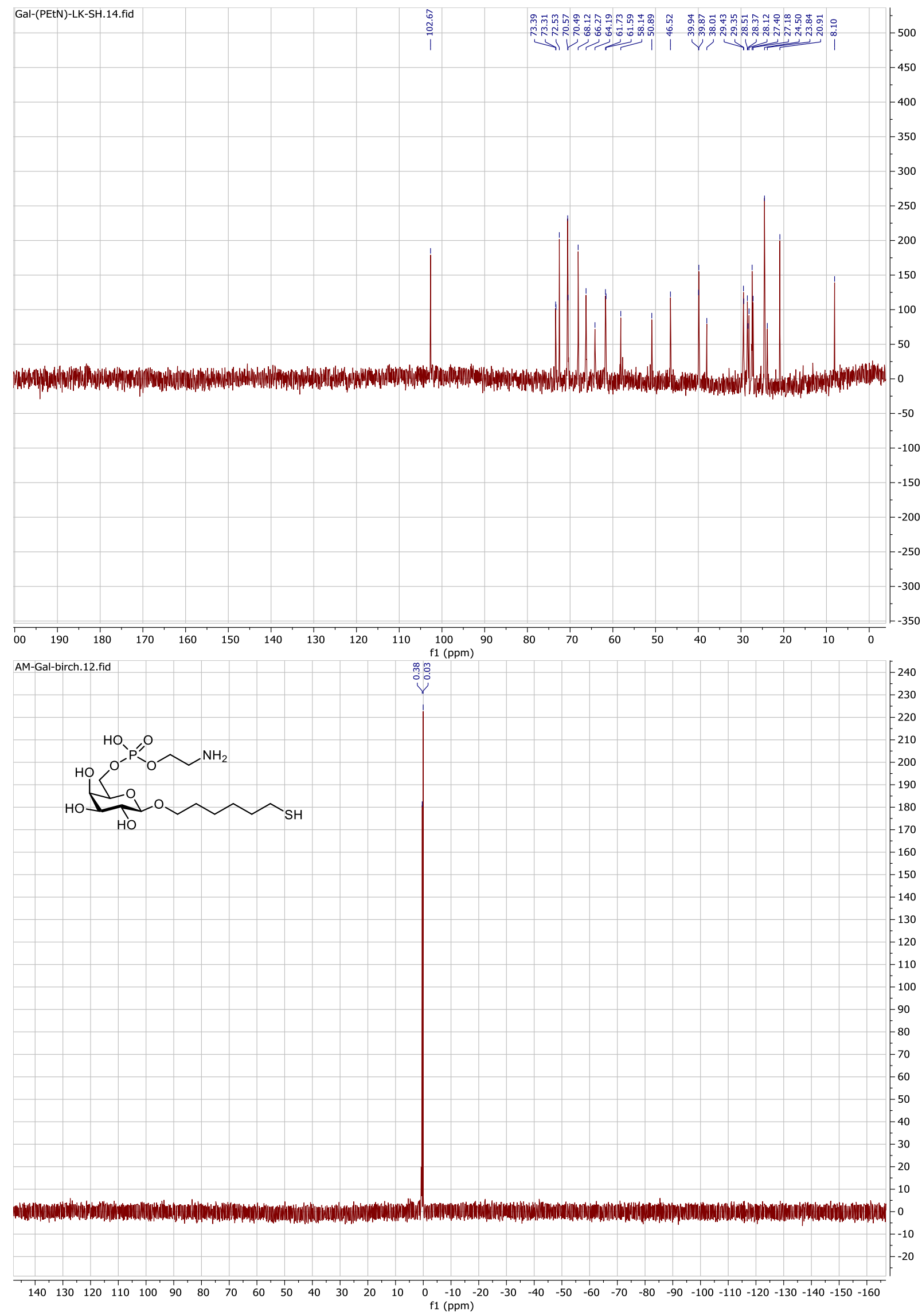




\section{References}

(1) Tsai, Y.-H.; Götze, S.; Vilotijevic, I.; Grube, M.; Silva, D. V.; Seeberger, P. H. (2013) A general and convergent synthesis of diverse glycosylphosphatidylinositol glycolipids. Chem. Sci., 4, 468-481.

(2) Kwon, Y. U.; Soucy, R. L.; Snyder, D. A.; Seeberger, P. H. (2005) Assembly of a series of malarial glycosylphosphatidylinositol anchor oligosaccharides. Chem. Eur. J., 11, 2493-2504.

(3) Tsai, Y.-H.; Götze, S.; Azzouz, N.; Hahm, H. S.; Seeberger, P. H.; Varon Silva, D. (2011) A General Method for Synthesis of GPI Anchors Illustrated by the Total Synthesis of the Low-MolecularWeight Antigen from Toxoplasma gondii. Angew. Chem. Int. Ed., 50, 9961-9964.

(4) Debenham, J. S.; Rodebaugh, R.; Fraser-Reid, B. (1997) TCP- and Phthalimide-Protected nPentenyl Glucosaminide Precursors for the Synthesis of Nodulation Factors As Illustrated by the Total Synthesis of NodRf-III (C18:1, MeFuc). J. Org. Chem., 62, 4591-4600.

(5) Tsai, Y.-H.; Gotze, S.; Vilotijevic, I.; Grube, M.; Varon Silva, D.; Seeberger, P. H. (2013) A general and convergent synthesis of diverse glycosylphosphatidylinositol glycolipids. Chem. Sci., 4, 468481.

(6) Grube, M.; Lee, B. Y.; Garg, M.; Michel, D.; Vilotijevic, I.; Malik, A.; Seeberger, P. H.; Silva, D. V. (2018) Synthesis of Galactosylated Glycosylphosphatidylinositol Derivatives from Trypanosoma brucei. Chem. Eur. J., 24, 3271-3282. 